INL EXT-20-58495

Revision 0

\title{
Non-destructive Examinations of ATF-2 Baseline Rodlets
}

Fabiola Cappia

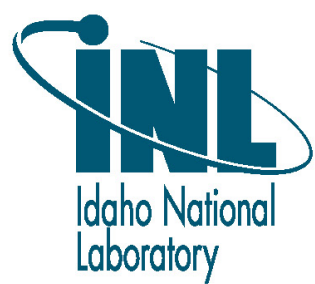




\section{DISCLAIMER}

Neither the U.S. Government nor any agency thereof, nor any of their employees, makes any warranty, expressed or implied, or assumes any legal liability or responsibility for the accuracy, completeness, or usefulness, of any information, apparatus, product, or process disclosed, or represents that its use would not infringe privately owned rights.

References herein to any specific commercial product, process, or service by trade name, trade mark, manufacturer, or otherwise, does not necessarily constitute or imply its endorsement, recommendation, or favoring by the U.S. Government or any agency thereof. The views and opinions of authors expressed herein do not necessarily state or reflect those of the U.S. Government or any agency thereof. Being provided this document, directly or indirectly, shall not be construed to constitute a governmental export license or authorization. 
INL EXT-20-58495

Revision 0

\title{
Non-destructive Examinations of ATF-2 Baseline Rodlets
}

\author{
Fabiola Cappia
}

June 2020

\section{Idaho National Laboratory \\ Characterization and Advanced PIE Division \\ Idaho Falls, Idaho 83415}

http://www.inl.gov

\author{
Prepared for the \\ U.S. Department of Energy \\ Office of Nuclear Energy \\ Under DOE Idaho Operations Office \\ Contract DE-AC07-05ID14517
}


Page intentionally left blank 


\section{SUMMARY}

This report contains the results of non-destructive examinations of Accident Tolerant Fuels 2 (ATF-2) rodlets irradiated in the Advanced Test Reactor (ATR) Loop 2A. The experiment is part of the U.S. Department of Energy Nuclear Technology Research and Development (NTRD) program's Advanced Fuels Campaign (AFC). The rodlets were composed of $\mathrm{UO}_{2}$ pellets and $\mathrm{Zr}-4$ cladding. The data have been collected to provide baseline Post-irradiation Examination (PIE) data to support future testing in Transient Reactor Test (TREAT) facility and to have performance data against which the data of the ATF concepts can be compared directly. The analyses performed included: visual examinations, axial gamma scanning, neutron radiography and profilometry. All the data collected showed a performance consistent with expectations for this fuel system at low burnup. 
Page intentionally left blank 


\section{ACKNOWLEDGEMENTS}

The author would like to acknowledge the operators and engineers of the Hot Fuel Examination Facility (HFEF) who executed the PIE. I would like to thank

B. Curnutt for fruitful discussion on the neutronic calculations, which was crucial for PIE data interpretation. I am also indebted to K. Williams, J. Skinner,

E. Beverly and all the operation and radioprotection staff of HFEF for technical and administrative support for this project. 
Page intentionally left blank 


\section{CONTENTS}

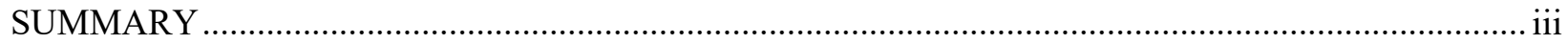

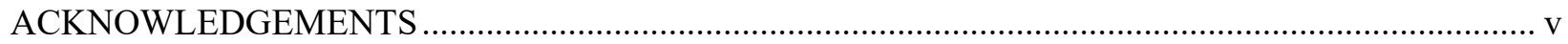

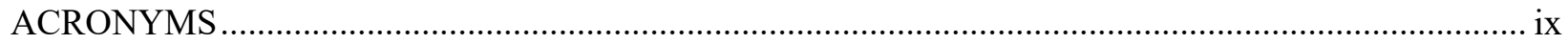

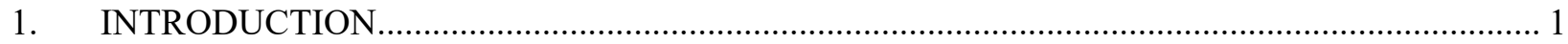

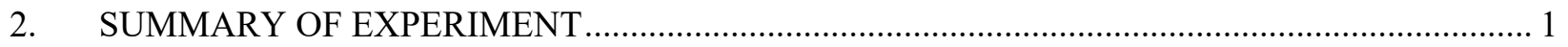

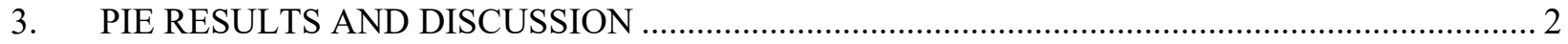

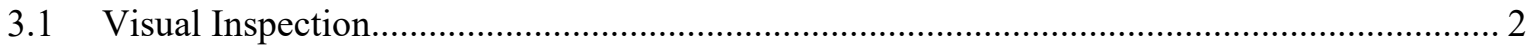

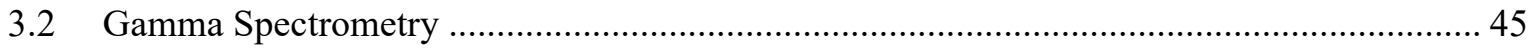

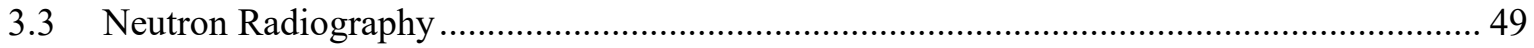

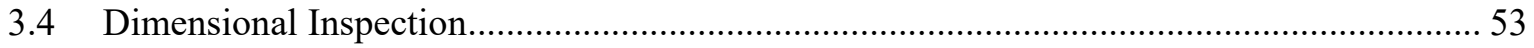

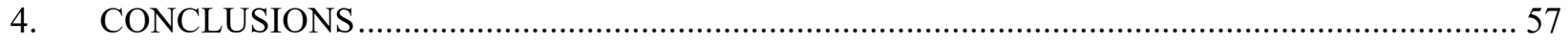

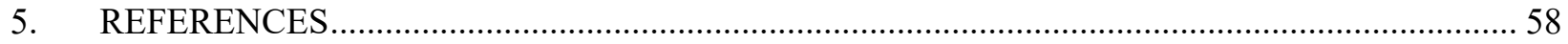

\section{FIGURES}

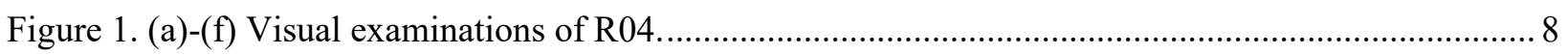

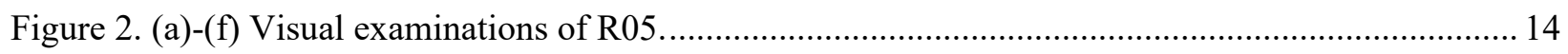

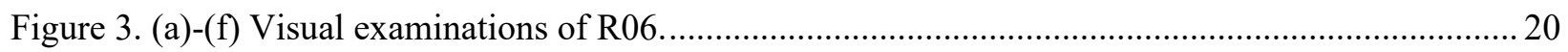

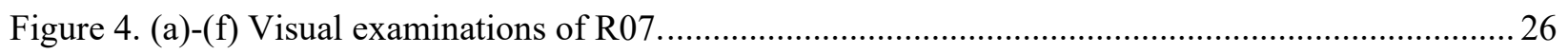

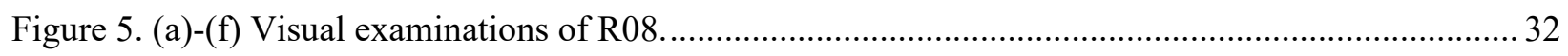

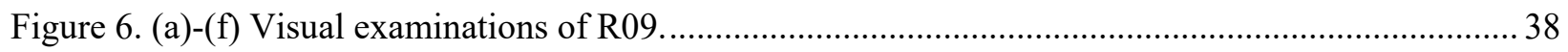

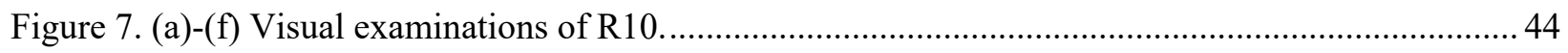

Figure 8: Axial gamma scan profile of selected fission products. ...................................................... 49

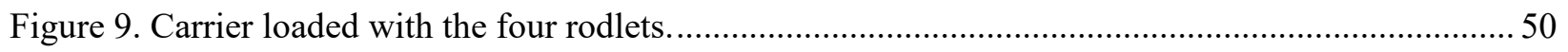

Figure 10. (a) Thermal and (b) epithermal neutron radiography of the rodlets at viewing angle $0^{\circ}$..........51

Figure 11. (a) Thermal and (b) epithermal neutron radiography of the rodlets at viewing angle $120^{\circ}$

Figure 12. (a) Thermal and (b) epithermal neutron radiography of the rodlets at viewing angle $240^{\circ}$

Figure 13. R04 cladding measured outer diameter (black circles). The solid red line represents the nominal diameter, while the dashed lines indicate the fabrication tolerance...

Figure 14. R06 cladding measured outer diameter (black circles). The solid red line represents the nominal diameter, while the dashed lines indicate the fabrication tolerance...

Figure 15. R08 cladding measured outer diameter (black circles). The solid red line represents the nominal diameter, while the dashed lines indicate the fabrication tolerance. 
Figure 16. R10 cladding measured outer diameter (black circles). The solid red line represents the nominal diameter, while the dashed lines indicate the fabrication tolerance.

\section{TABLES}

Table 1. Rodlet IDs and predicted discharge burnup..................................................................... 1 


\section{ACRONYMS}

AFC Advanced Fuels Campaign

AOO anticipated operational occurrence

ATF Accident Tolerant Fuels

ATR Advanced Test Reactor

HFEF Hot Fuel Examination Facility

INL Idaho National Laboratory

LWR Light Water Reactor

NRAD Neutron Radiography (Reactor)

NTRD Nuclear Technology Research and Development

PGS Precision Gamma Screening

PIE Postirradiation Examination

TREAT Transient Reactor Test (facility) 
Page intentionally left blank 


\section{Non-destructive Examinations of ATF-2 Baseline Rodlets}

\section{INTRODUCTION}

A fueled experiment that is part of the U.S. Department of Energy Nuclear Technology Research and Development (NTRD) program's Advanced Fuels Campaign (AFC) is being conducted in the Advanced Test Reactor (ATR) Loop 2A. The main goal of the Accident Tolerant Fuels (ATF) campaign is to demonstrate the improved performance of new fuel and cladding concepts with respect to the standard Light Water Reactor (LWR) fuels, i.e., $\mathrm{UO}_{2}$ and zircaloy. The scope of the ATF-2 experiments is to provide data regarding the performance of these new concepts under pressurized water reactor operating conditions. As part of the experiment series, a total of eight rodlets composed of standard $\mathrm{UO}_{2}$ pellets and $\mathrm{Zr}-4$ cladding have been irradiated in the experimental tier to provide the baseline data against which the performance of the new concepts will be compared.

Some of these rodlets will be part of the irradiation experiment ATF-R to be performed in the INL Transient Test Reactor (TREAT) facility [1]. The scope of the ATF-R experiment is to perform anticipated operational occurrence (AOO) transients and to evaluate whether rods that experienced a short duration of the dry-out event can be returned to service, and, if so, whether any penalties need to be taken in the safety analysis for fuel damage. Data characterizing the status of the rodlets before the transient testing are, therefore, needed to compare the post-transient results.

A total of seven rodlets was received at the Idaho National Laboratory (INL) Hot Fuel Examination Facility (HFEF) in February 2020. This report contains the results of non-destructive examinations of these baseline ATF-2 rodlets.

\section{SUMMARY OF EXPERIMENT}

The rodlets are nominally $15.24 \mathrm{~cm}$ ( 6 inches) long, composed of commercial $\mathrm{Zr}-4$ and $\mathrm{UO}_{2}$ pellets. The total length of the fuel stack was approximately $10 \mathrm{~cm}$ long (4 inches). The rodlet identification and the calculated discharge burnup are reported in Table 1. Further information regarding the irradiation conditions can be found in Ref. [2].

Table 1. Rodlet IDs and predicted discharge burnup.

\begin{tabular}{cc}
\hline Rodlet ID & Burnup (GWd/tHM) \\
\hline R-04 & 9.32 \\
R-05 & 9.27 \\
R-06 & 9.54 \\
R-07 & 9.23 \\
R-08 & 9.47 \\
R-09 & 9.37 \\
R-10 & 10.29 \\
\hline
\end{tabular}




\section{PIE RESULTS AND DISCUSSION}

PIEs were performed at the INL HFEF. Visual examinations and gamma scanning were conducted on all seven rodlets. In the second stage of the PIE, four rodlets have been chosen to continue for further non-destructive examination, specifically, a neutron radiography and profilometry. The results of these exams are summarized in the following sections.

\subsection{Visual Inspection}

A new holder for the visual examinations has been designed and fabricated in order to perform the visual inspections at known azimuthal orientations. A ruler was incorporated on the back of the holder as a length reference. The ruler is in metric units on the left side of the rodlet and imperial units on the right side of the rodlet. Photo documentation of the rodlets consisted in a digital picture spanning the entire rodlet length. Visual inspection was performed on the rodlets at six different azimuthal views, each $60^{\circ}$ apart. The photos taken through the cell window are shown in Figure 1 through Figure 7.

From the analysis of all the various viewing angles, it can be seen that the cladding surface of all rodlets presents several superficial scratches, either parallel to the rodlet axis or along a slightly diagonal direction. The rodlets were handled several times in the canal during tier re-organization. The rodlet holder has abrasive pin support separators in the shape of four strips that hold the rodlets in place in the tier. As they were pulled out for tier re-organization and shipping in the cask basket, abrasion is likely to have occurred, causing the observed shallow scratches. Occasionally, deeper scratches are present, such as the one visible in Figure $2 \mathrm{c}$ starting $\sim 5 \mathrm{~cm}$ from rodlet bottom and spanning along the rest of the rodlet height. Other examples can be seen in Figure 3a starting at the bottom of R06, on the right side of the viewing angle, and in Figure 7a. Patchy discoloration was observed at the top of R06 in Figure 3b, underneath the rodlet ID. The surface of R06 both in Figure 3d and e appears heterogeneous in color, with discontinuous opaque areas, likely an effect of oxidation. The surface of R08 in Figure 5c, of R09 in Figure $6 \mathrm{c}$ and d, and of R10 in Figure 7c-f shows analogous characteristics. A circular spot was observed at approximately $2.5 \mathrm{~cm}$ from bottom in R06 (Figure 3e). A similar, but smaller circular spot was observed in Figure $2 \mathrm{f}$ at similar height in R05, and in R08 at $11.4 \mathrm{~cm}$ from rodlet bottom (Figure 5a). A round bright spot can be also seen at the very bottom of R09 (Figure 6a). 


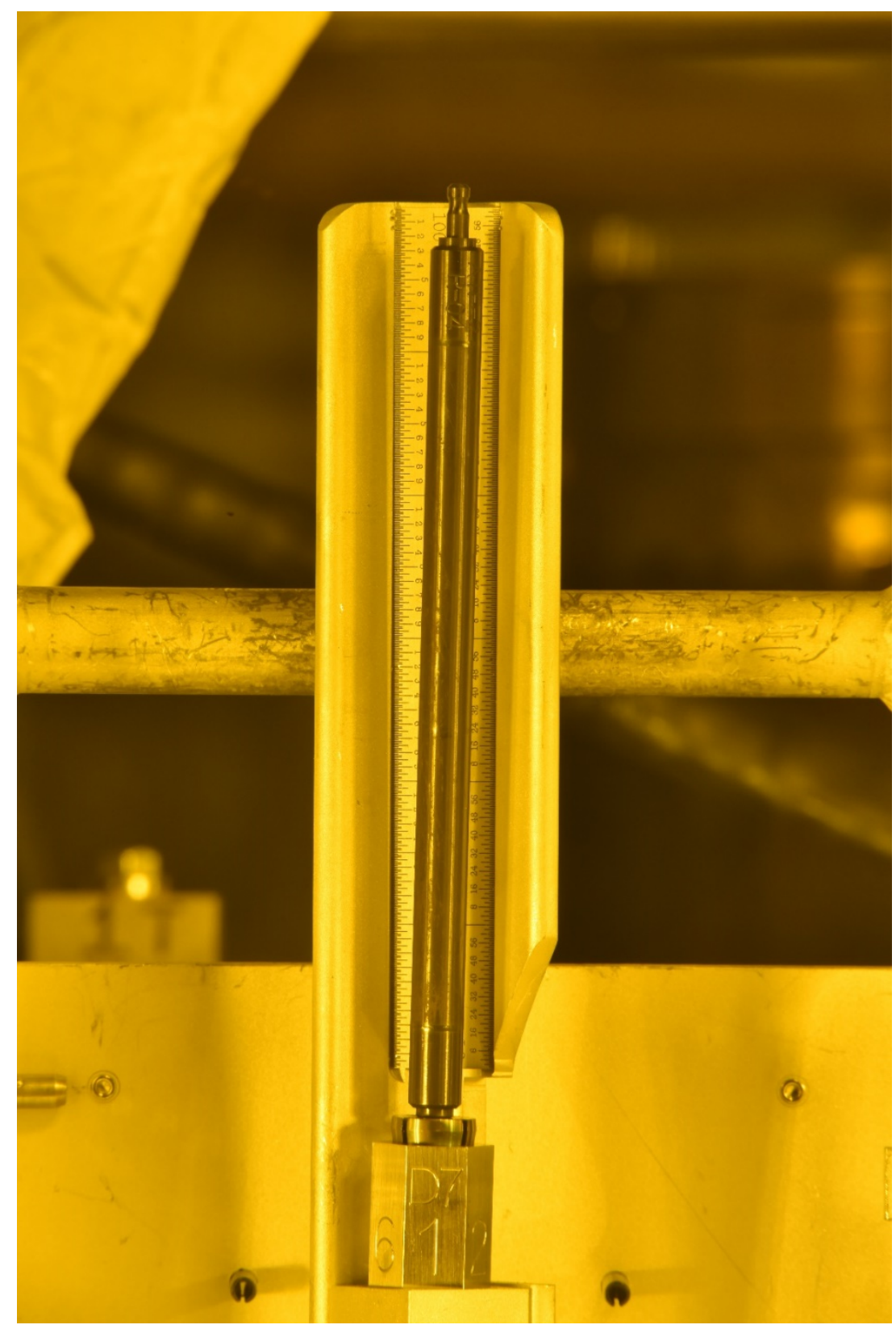

(a) 


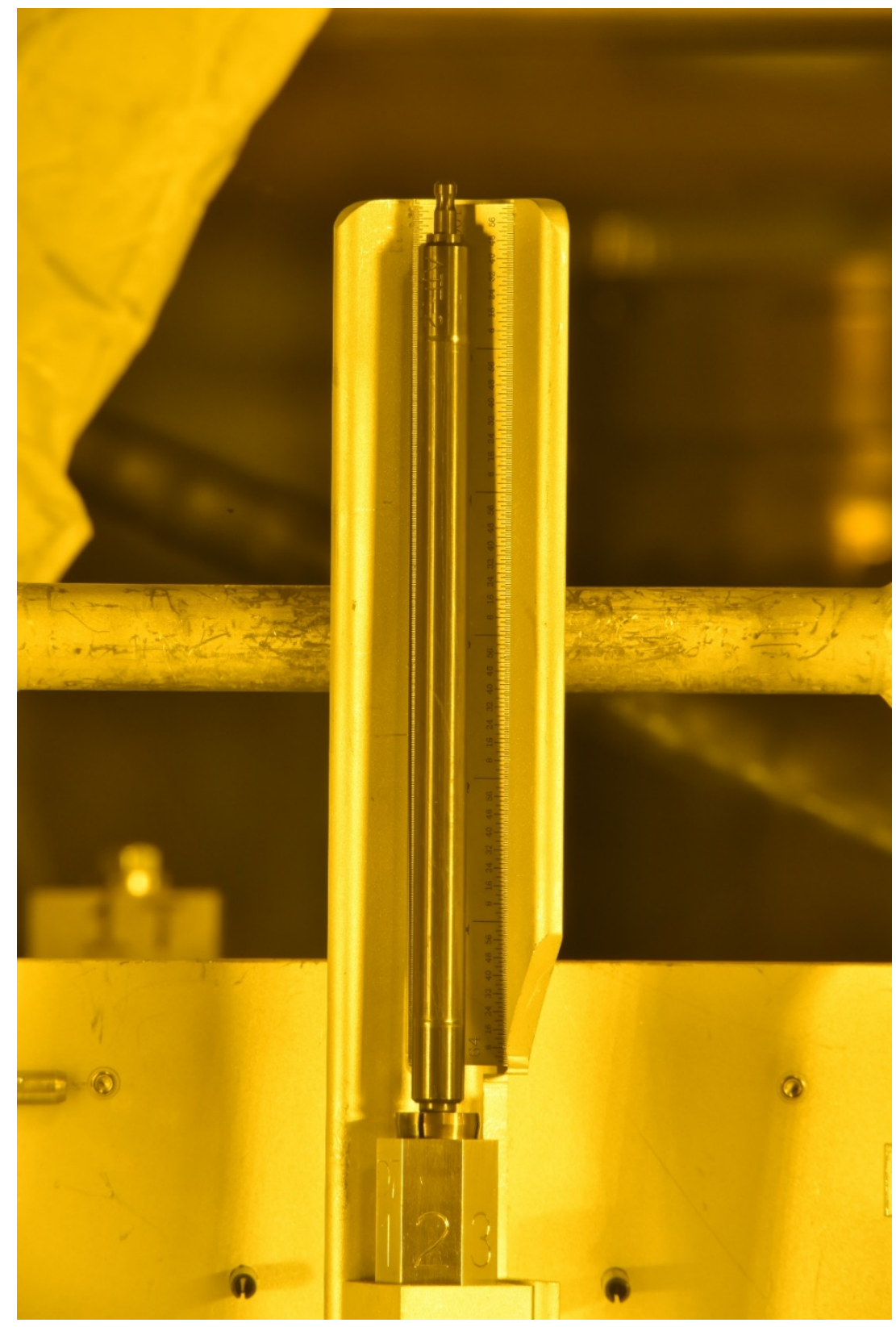

(b) 


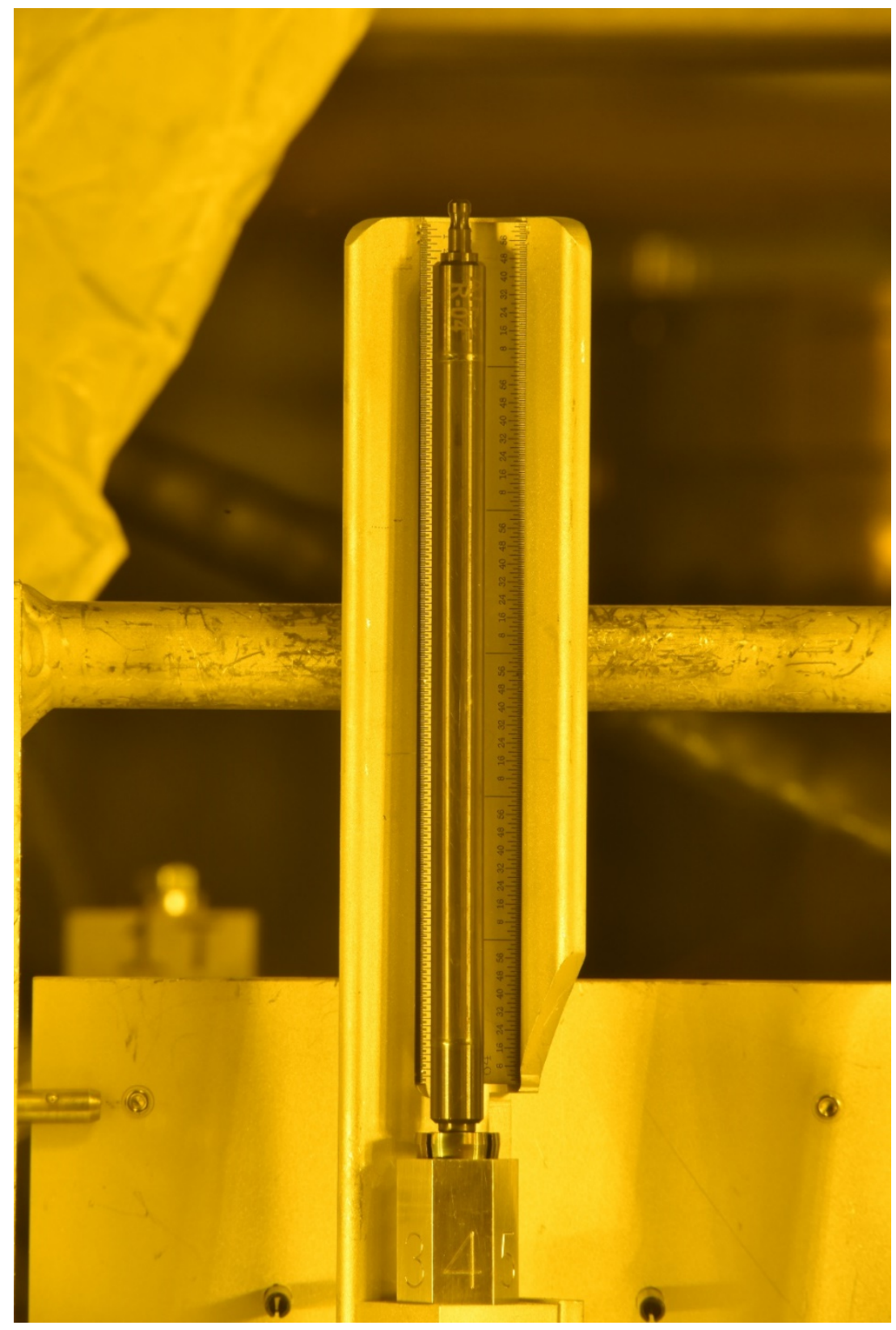

(c) 


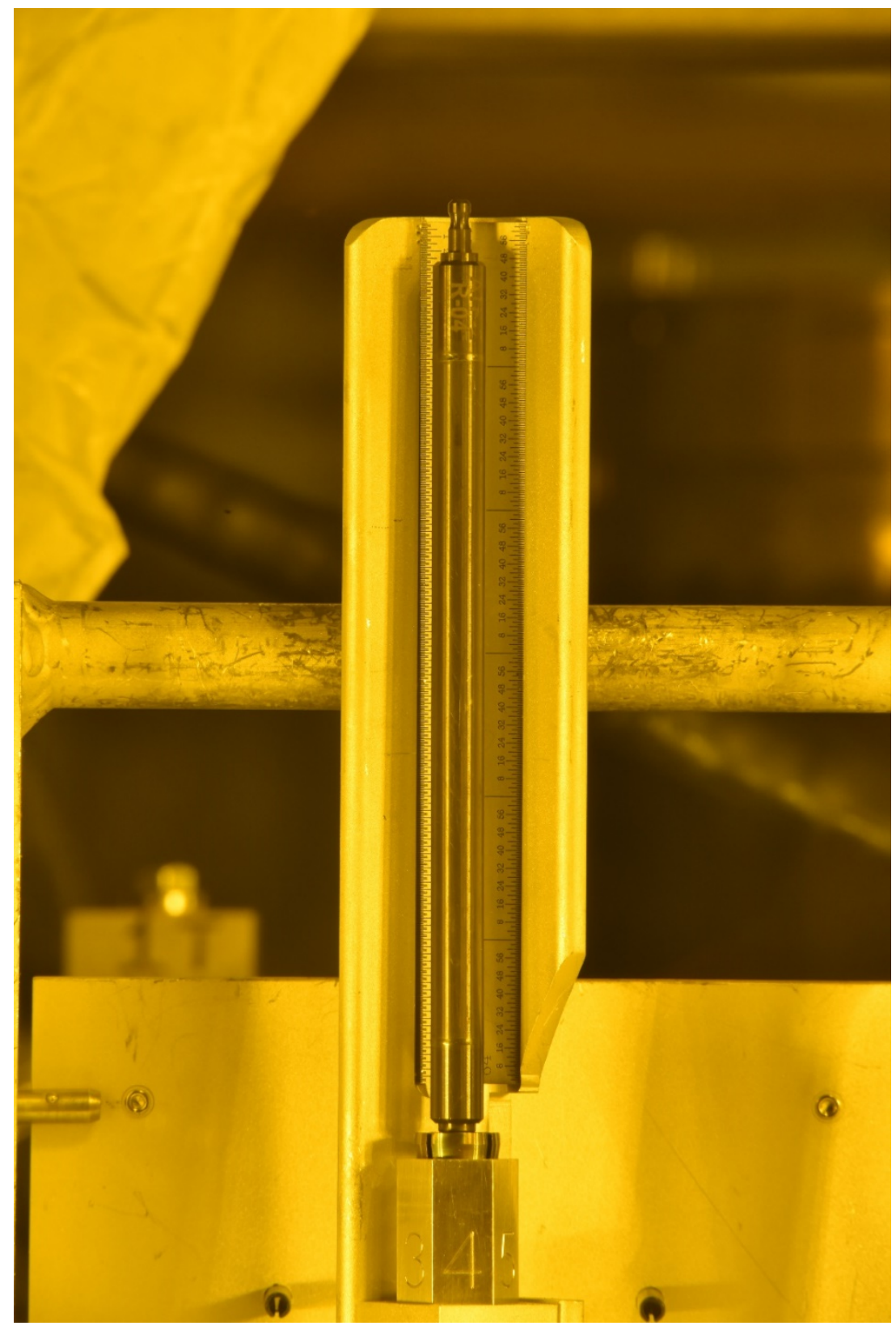

(d) 


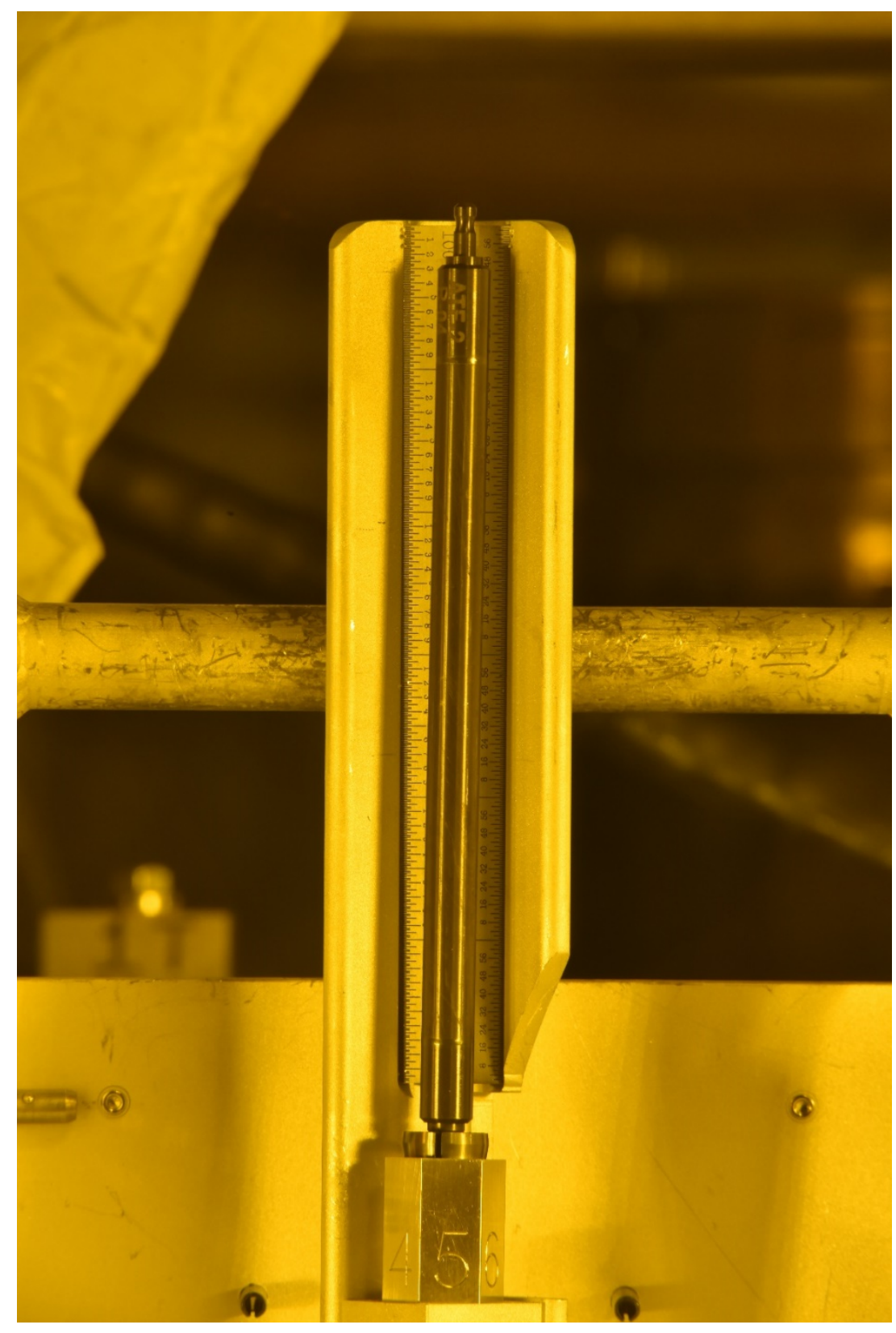

(e) 


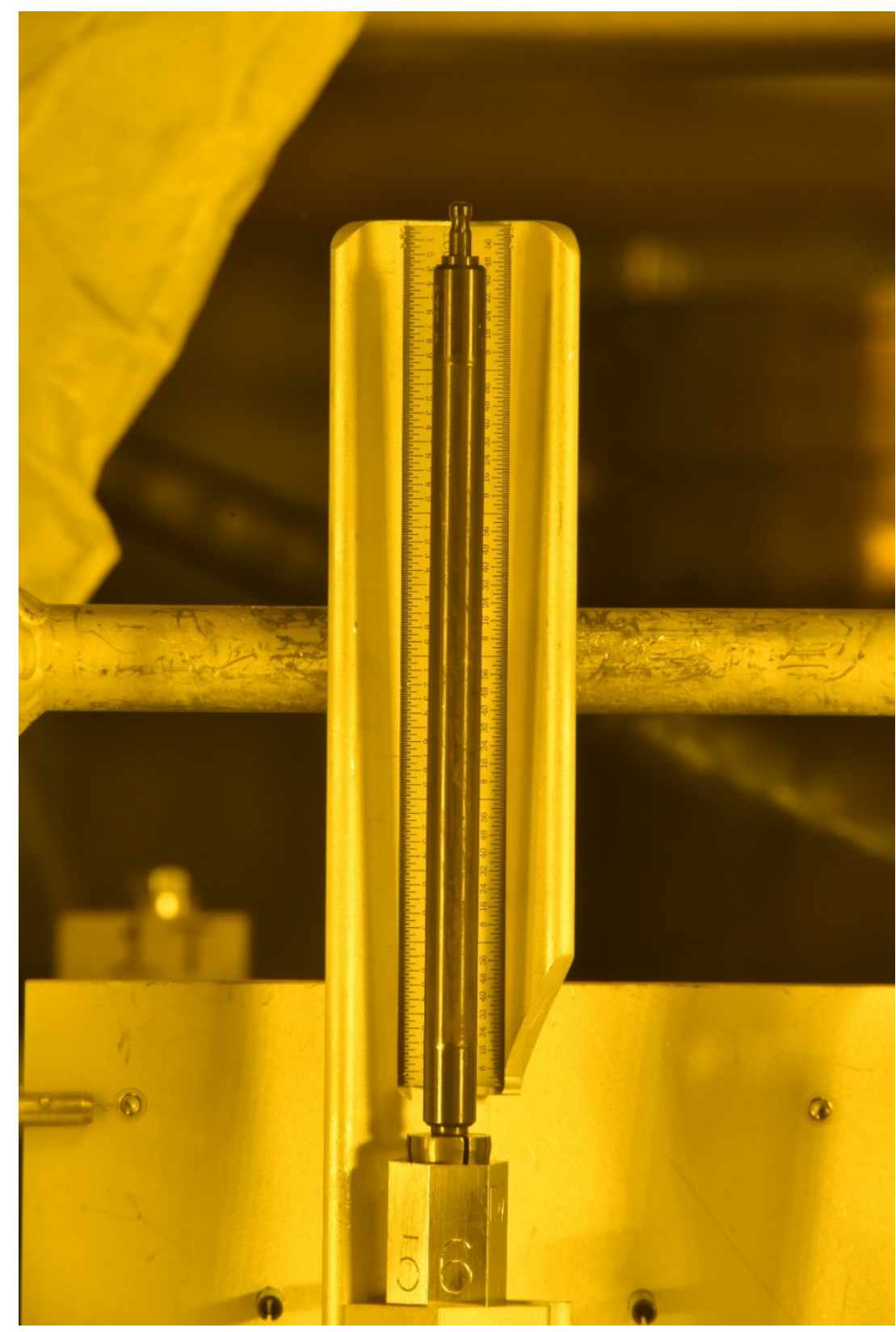

(f)

Figure 1. (a)-(f) Visual examinations of R04. 


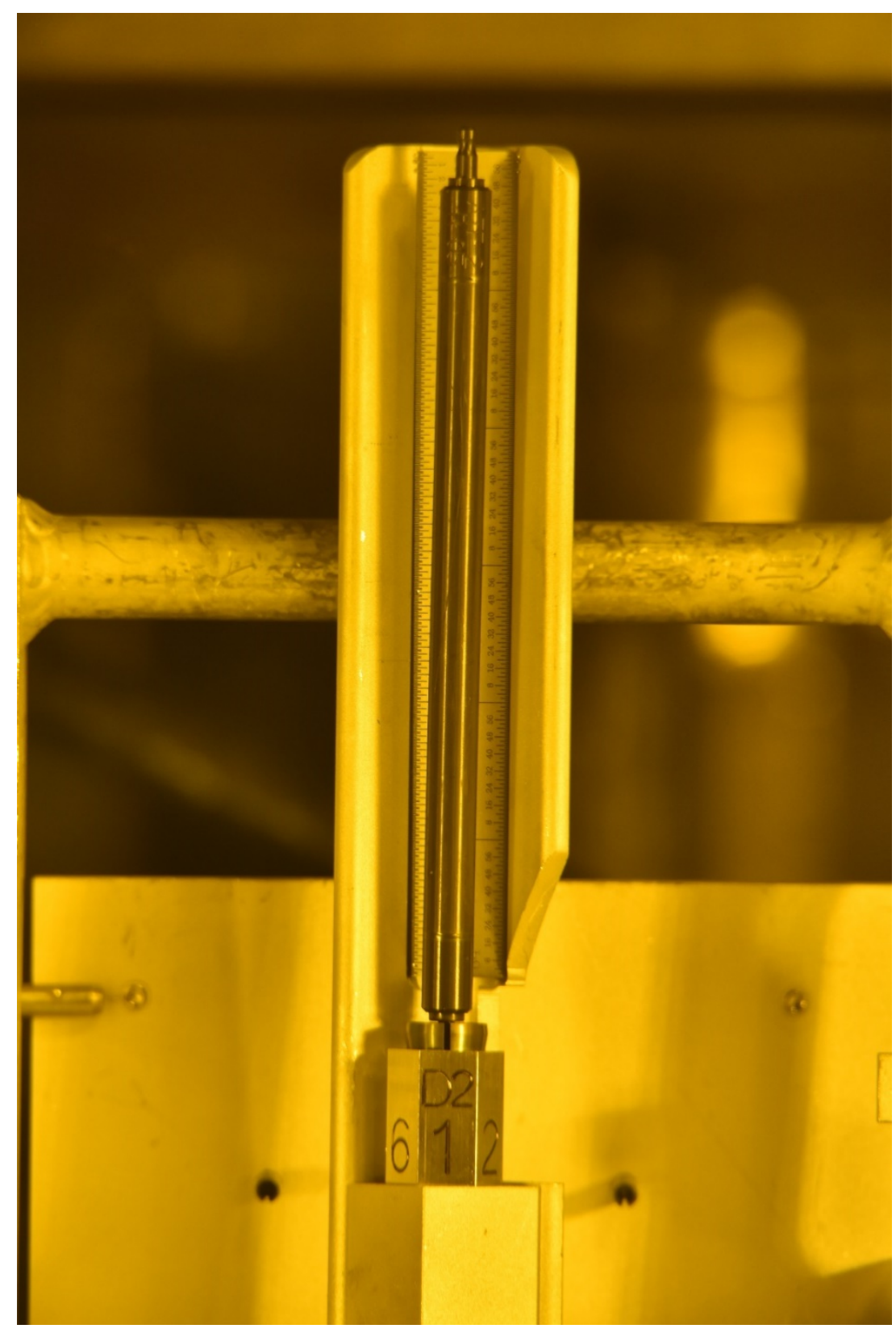

(a) 


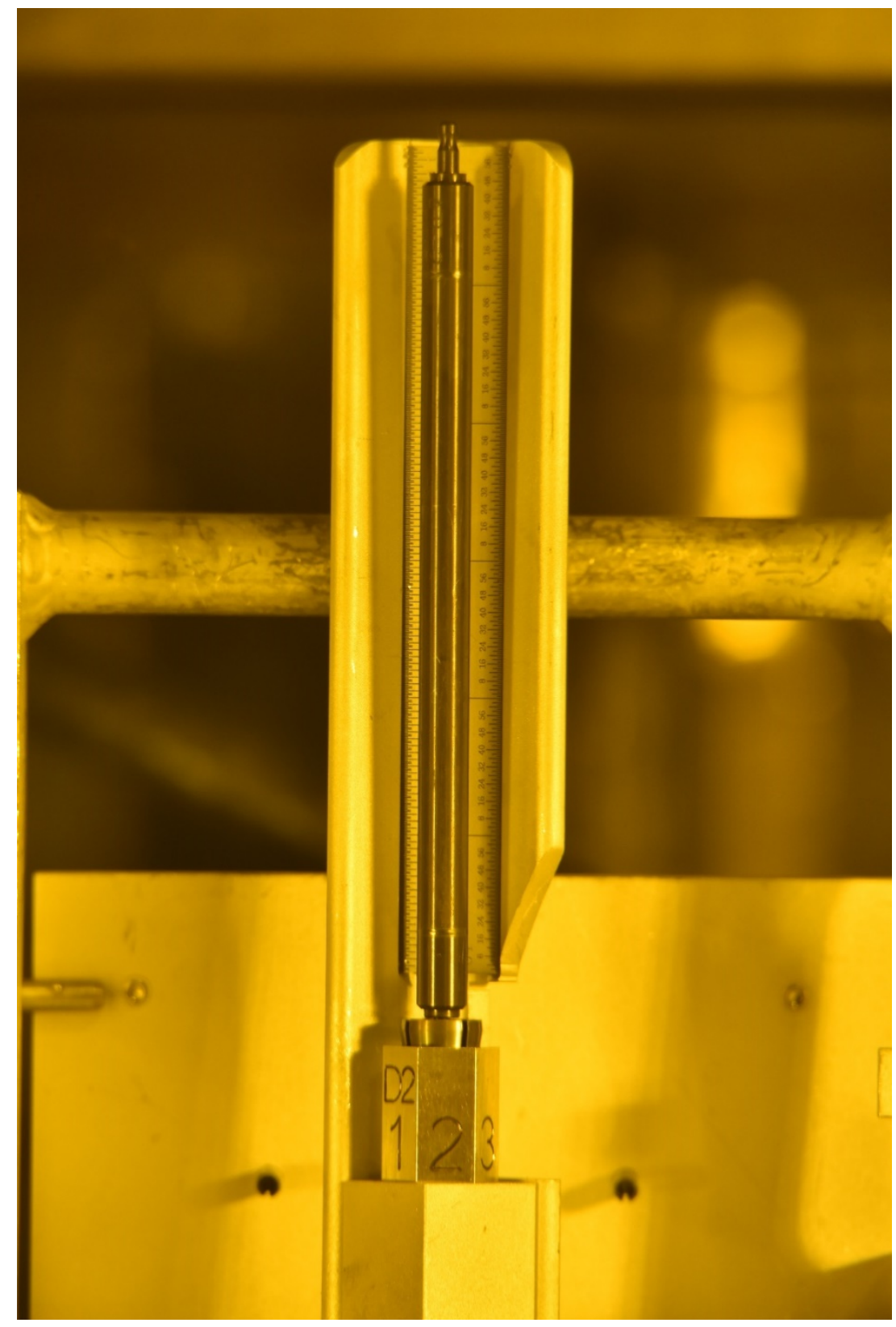

(b) 


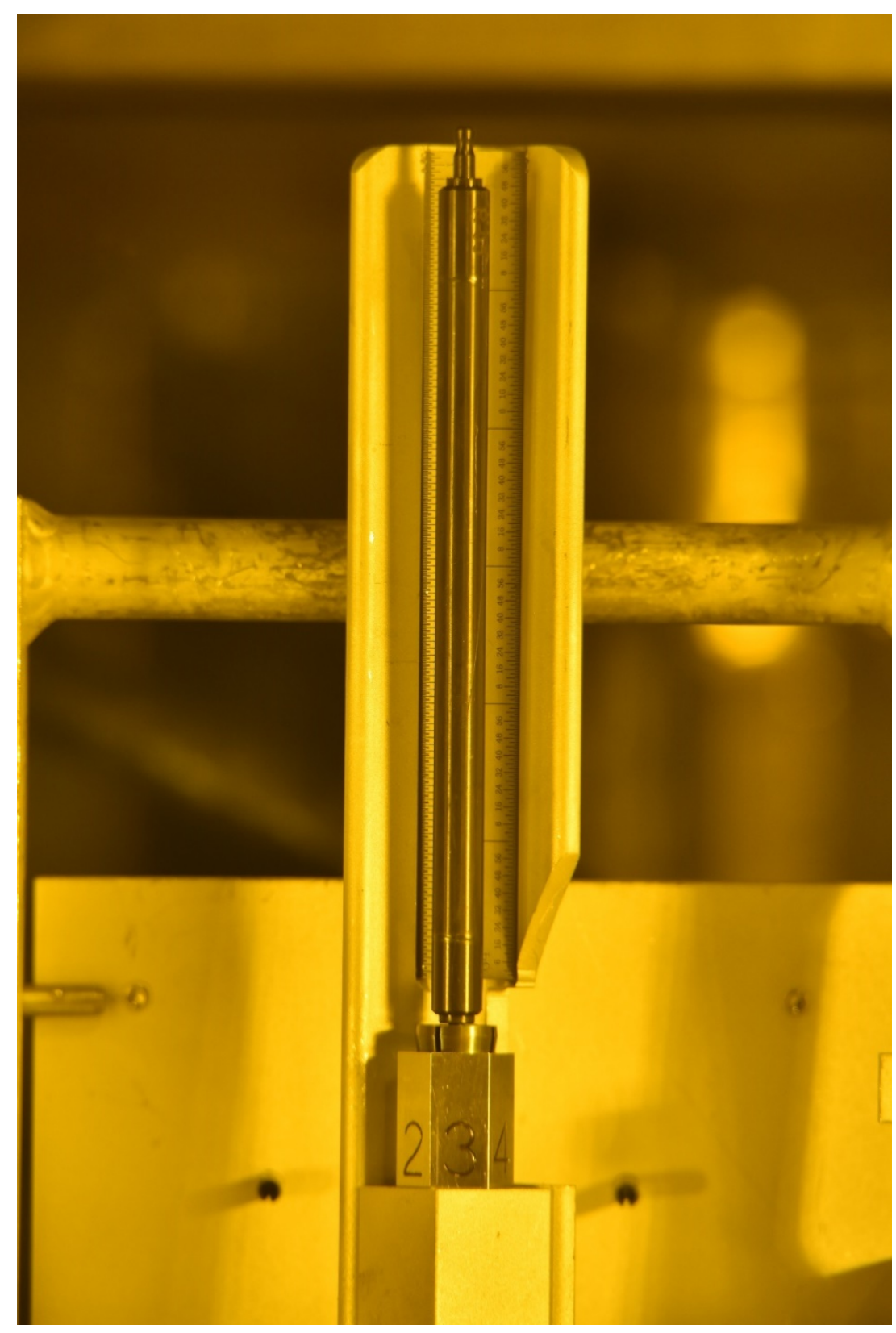

(c) 


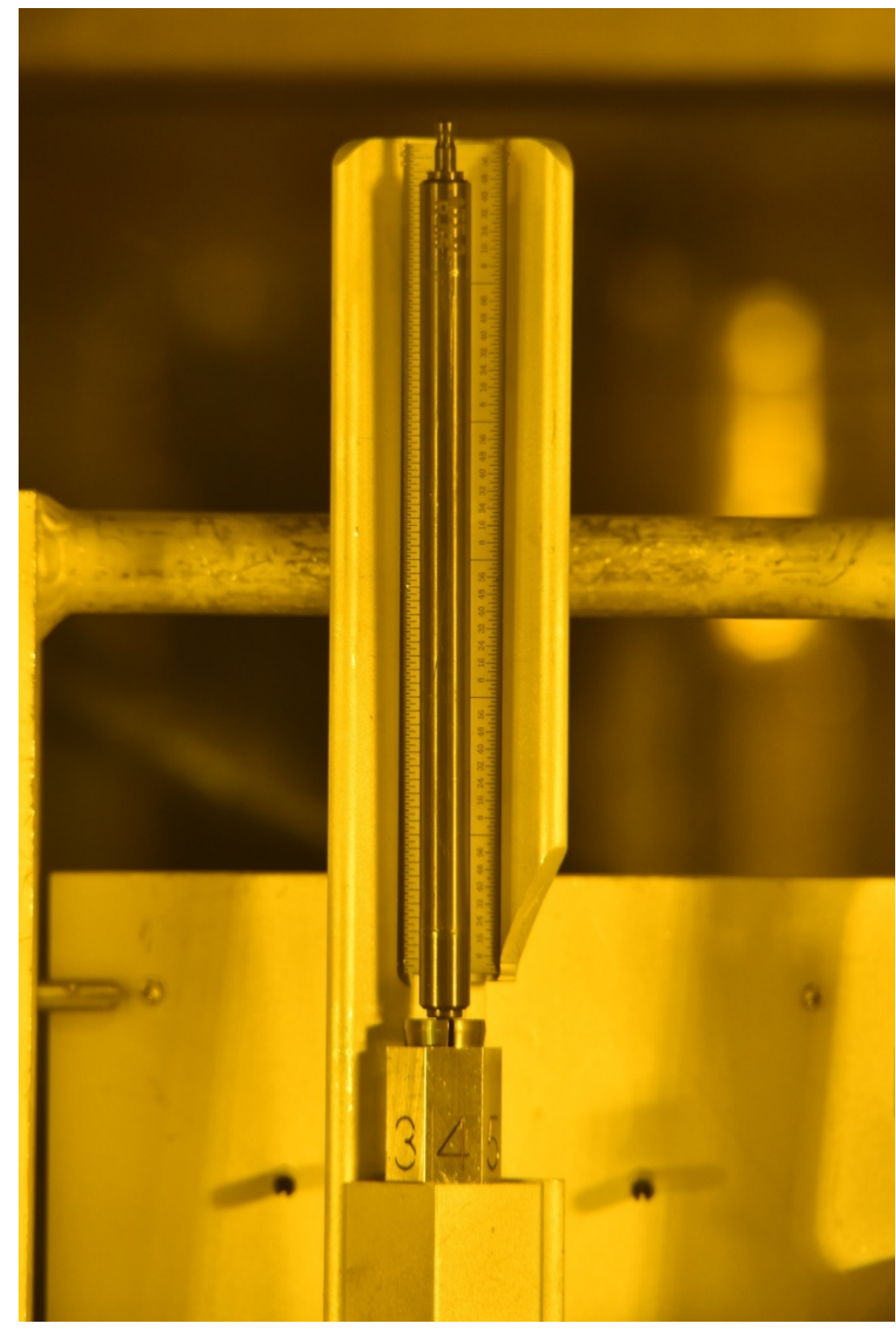

(d) 


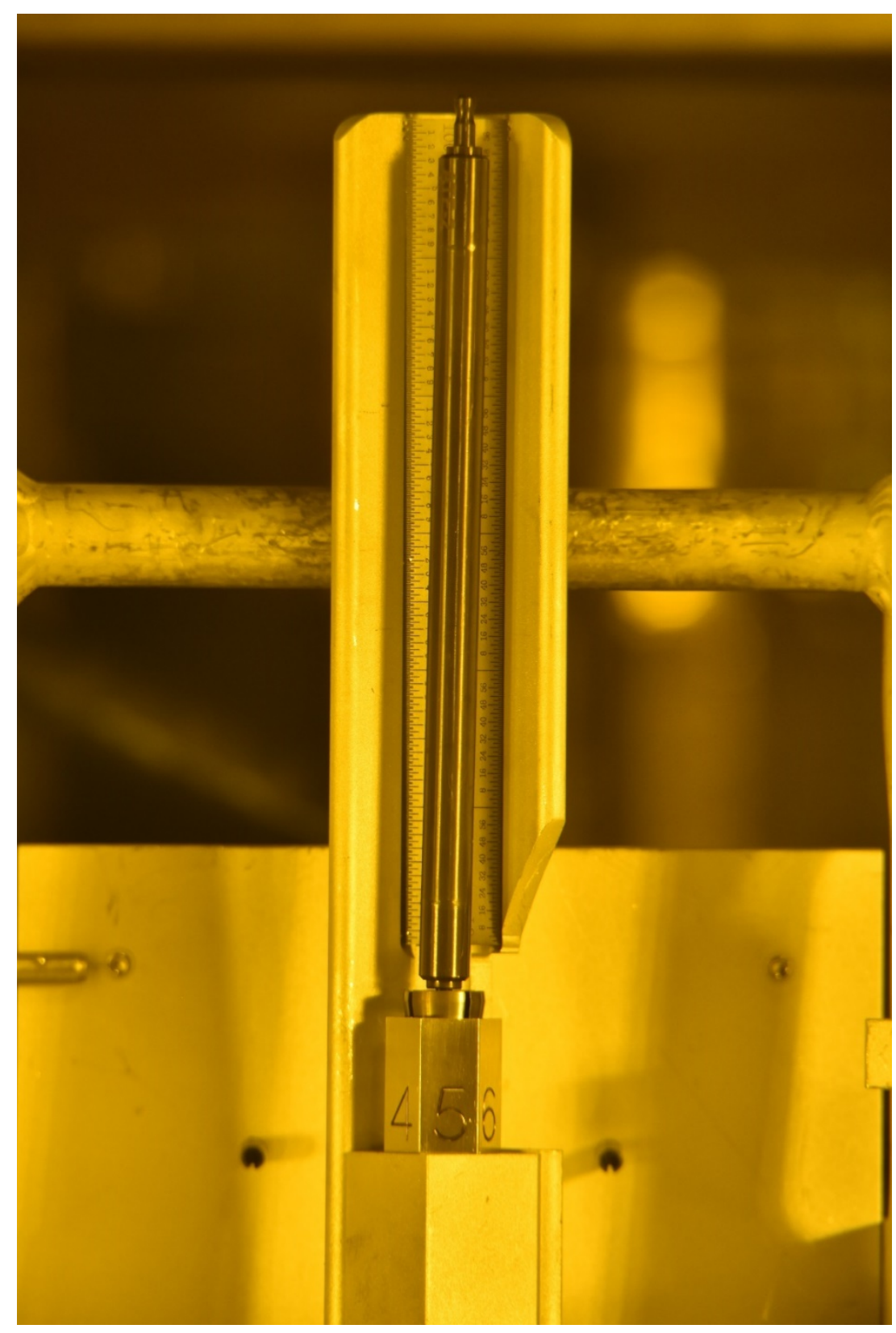

(e) 


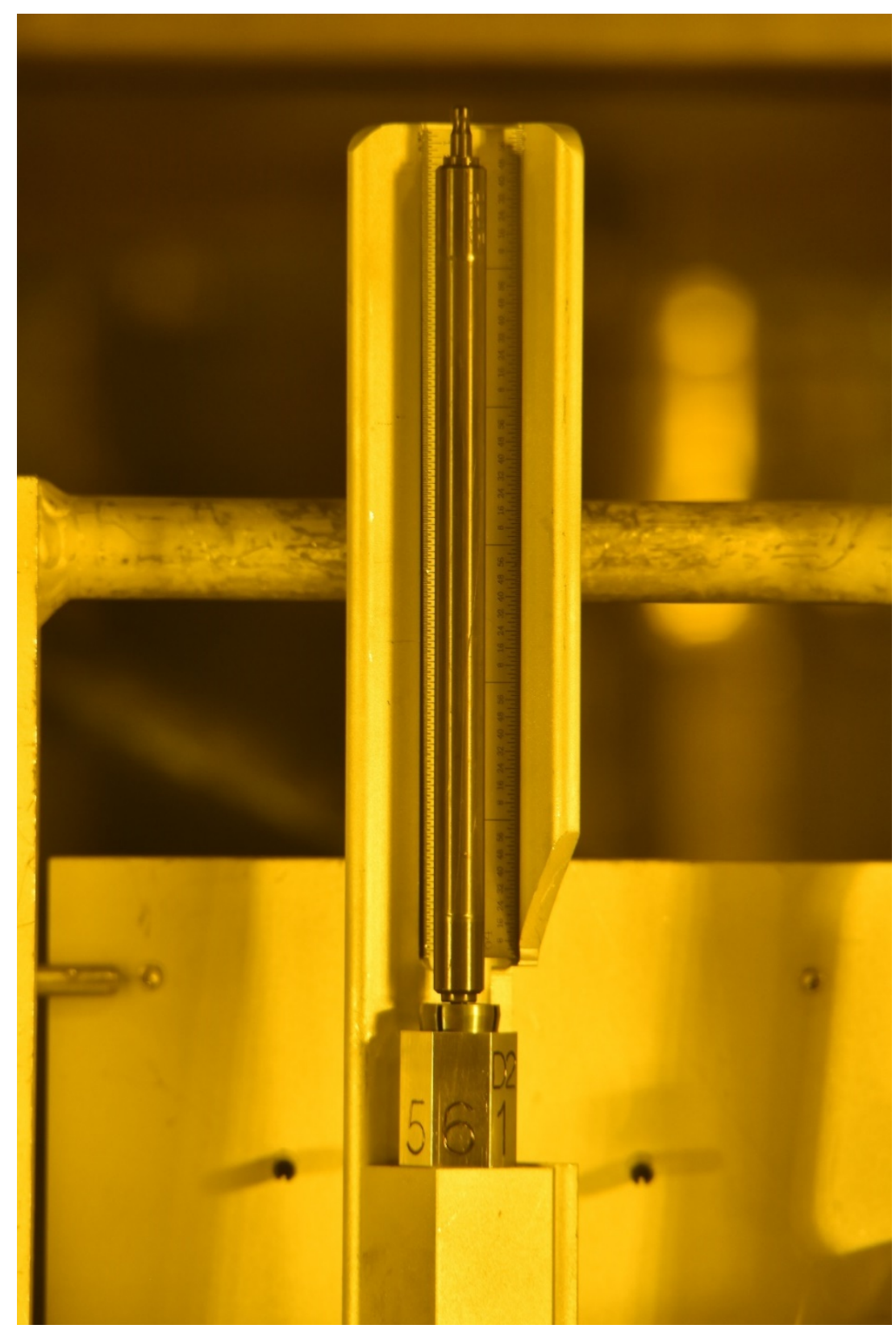

(f)

Figure 2. (a)-(f) Visual examinations of R05. 


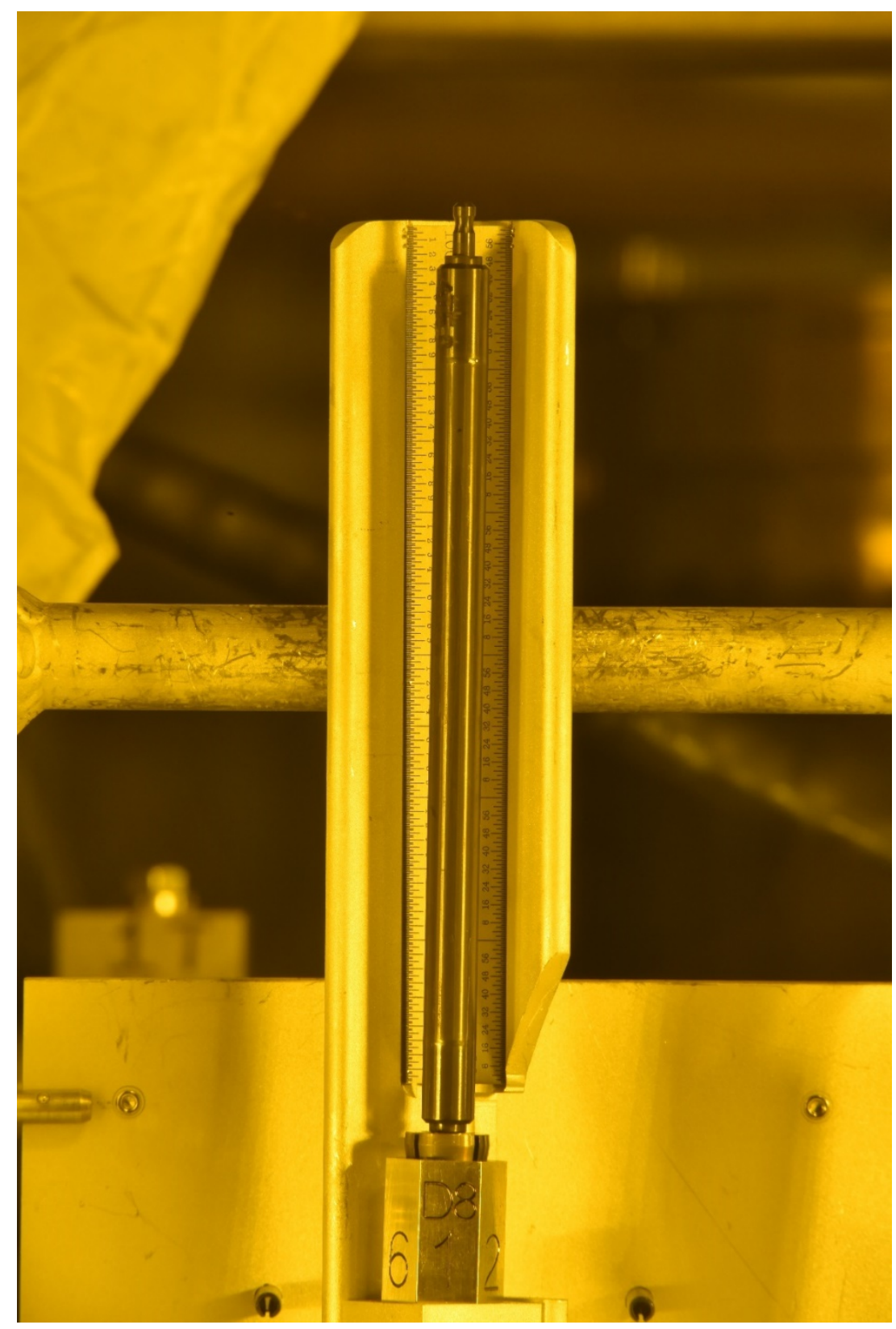

(a) 


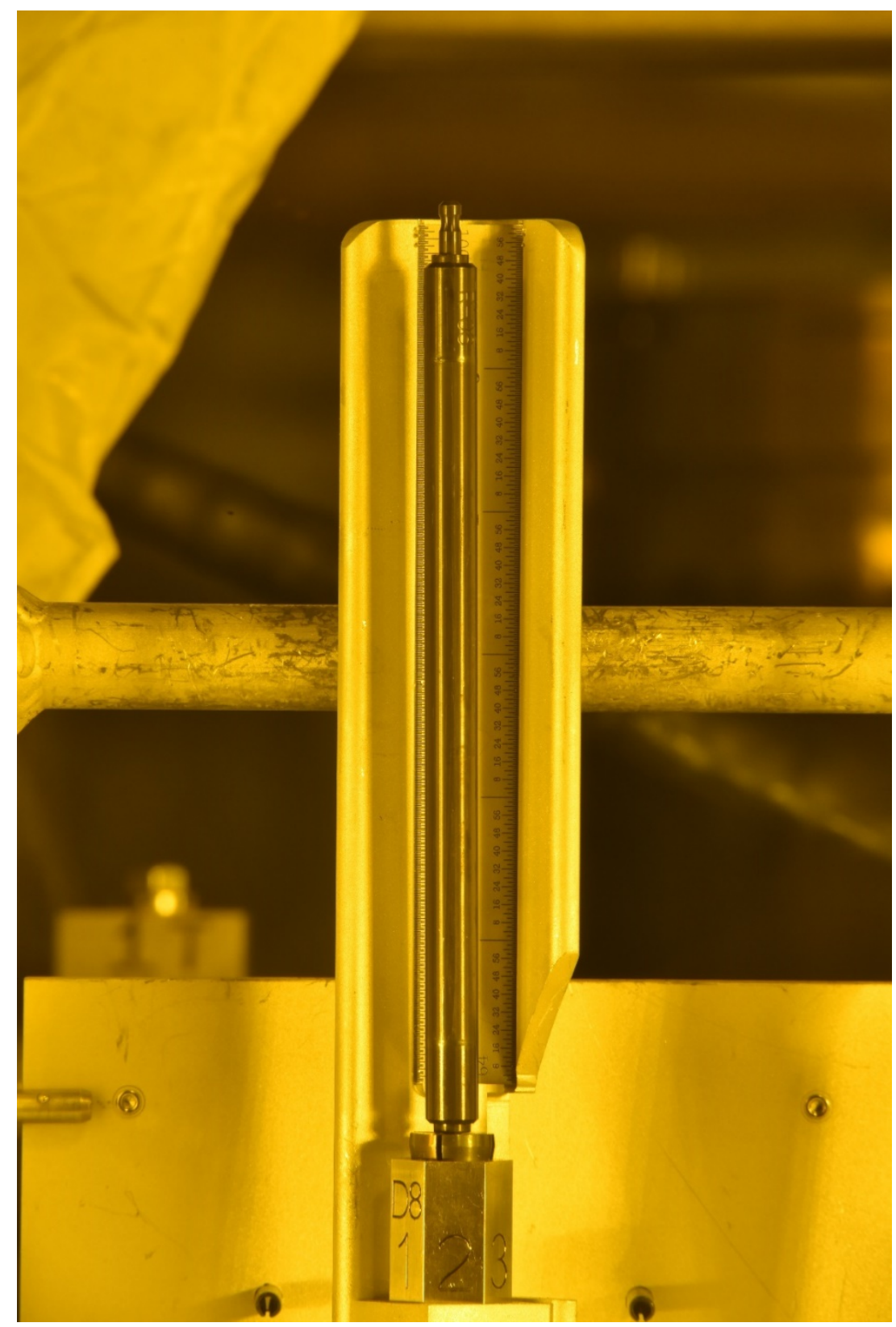

(b) 


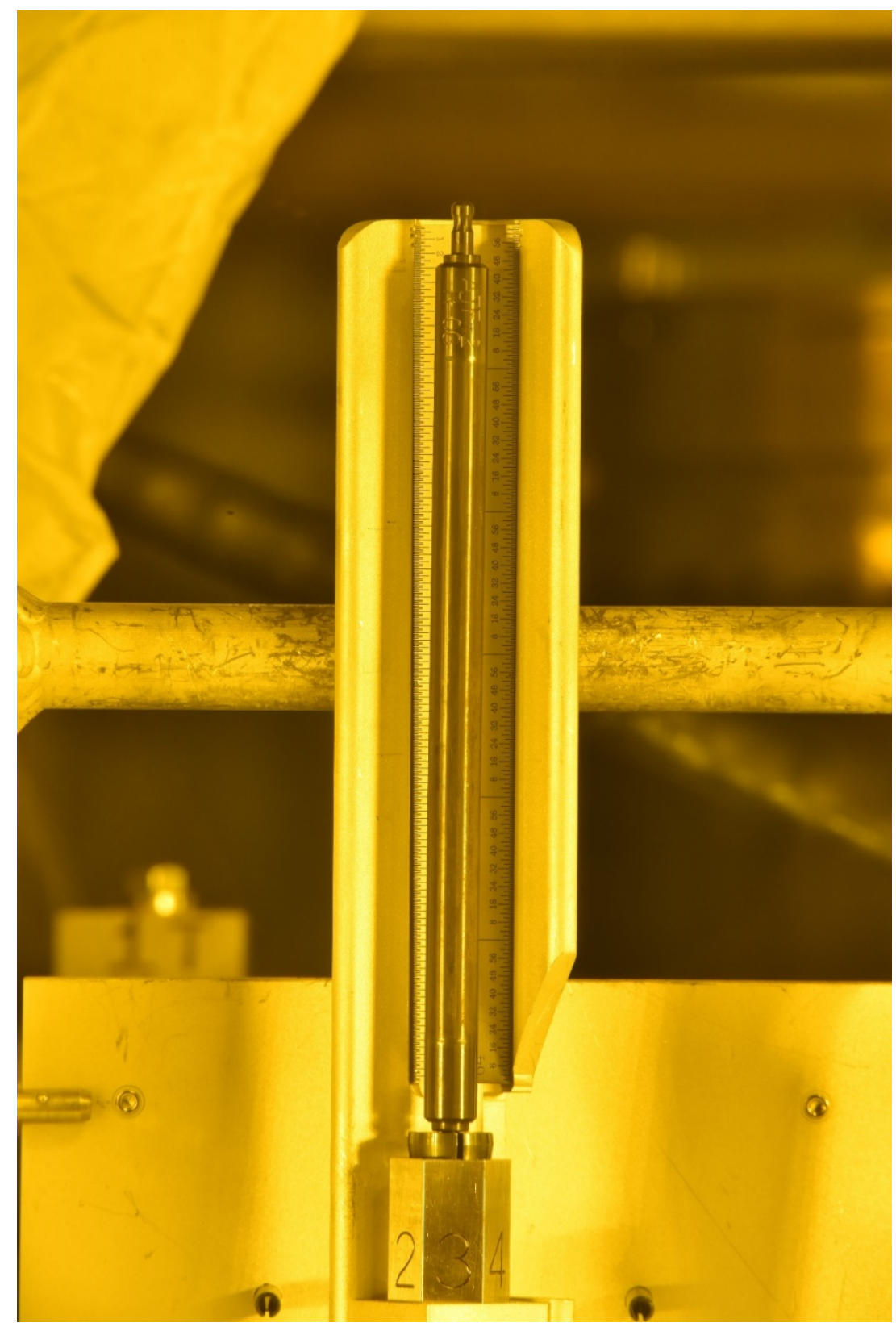

(c) 


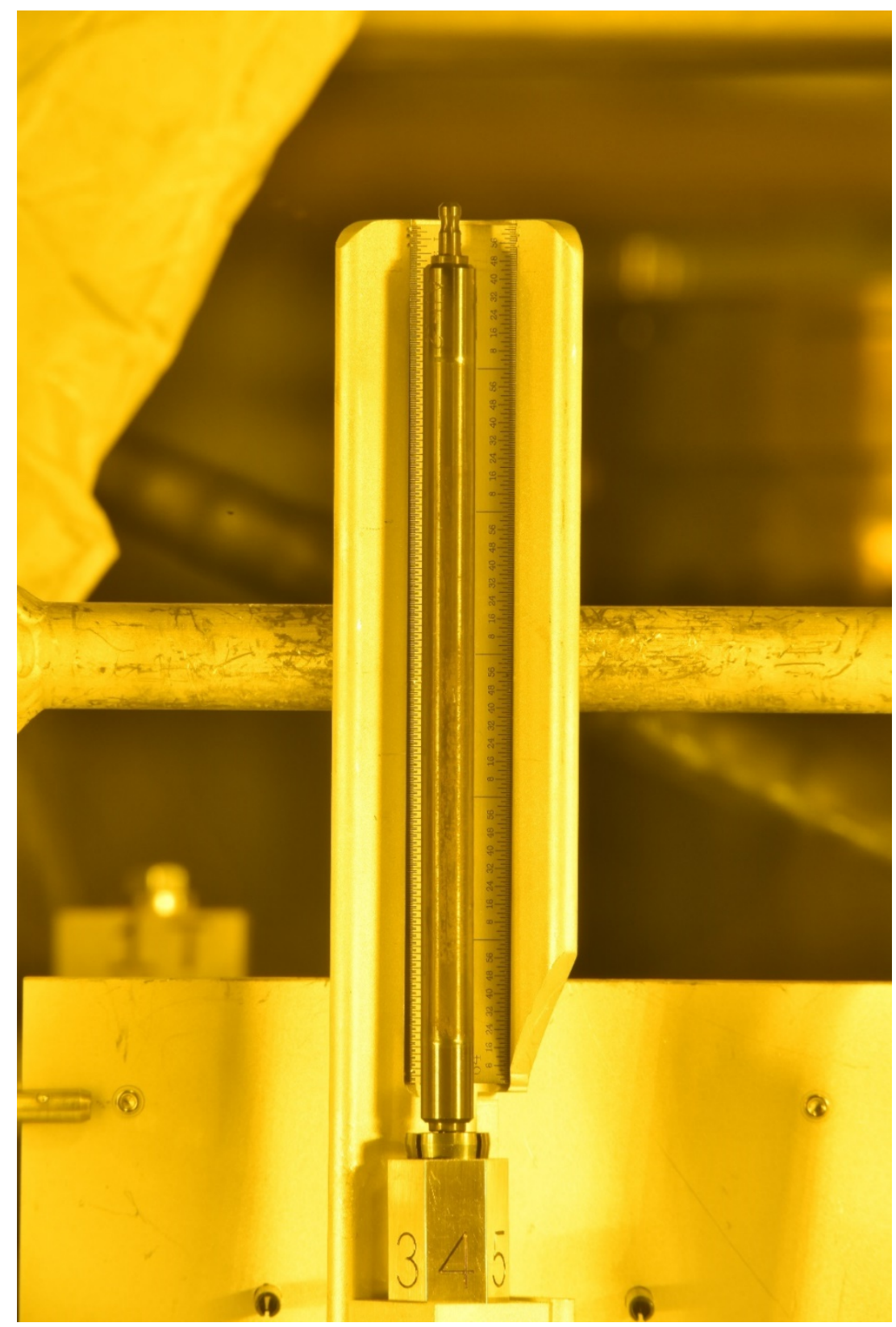

(d) 


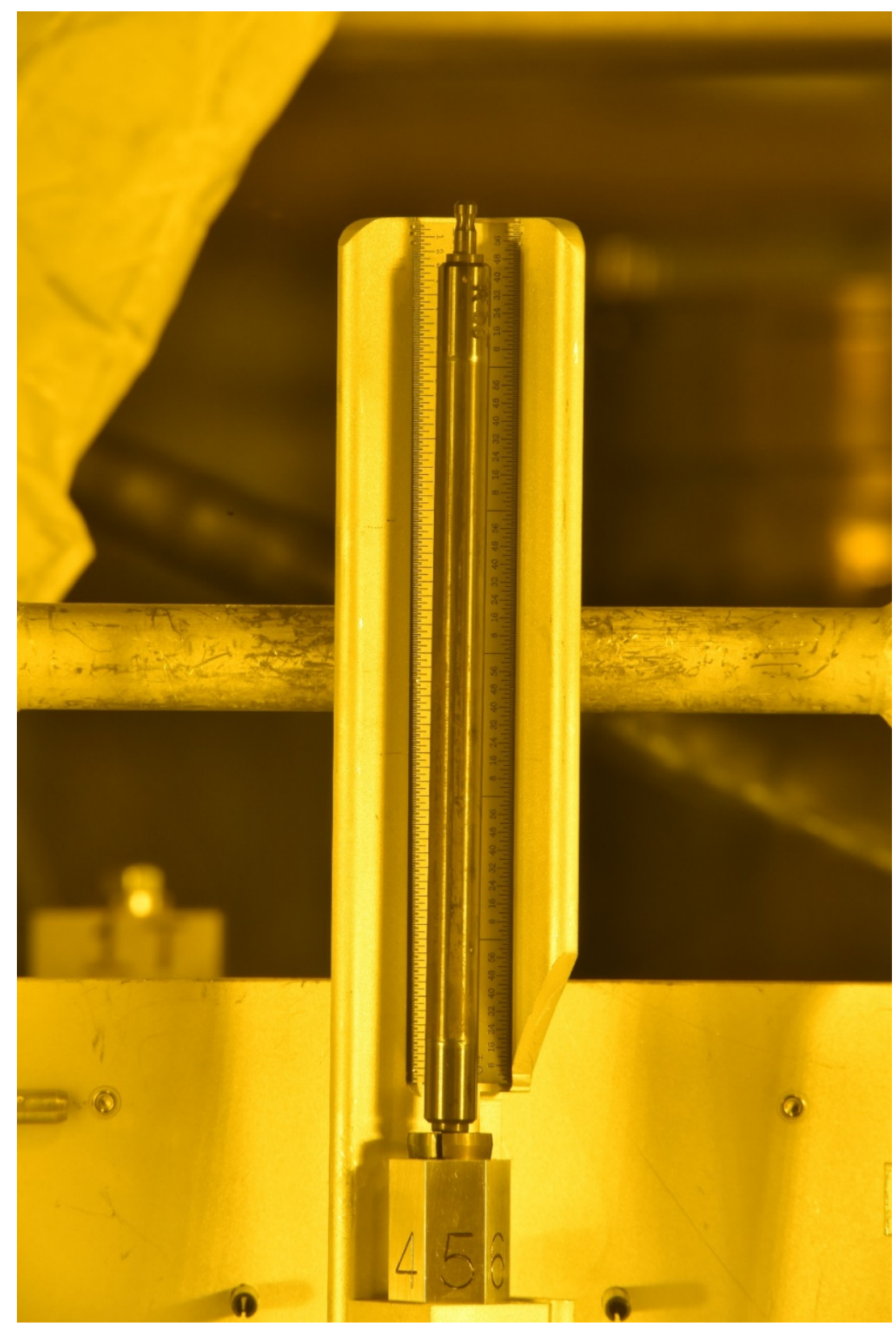

(e) 


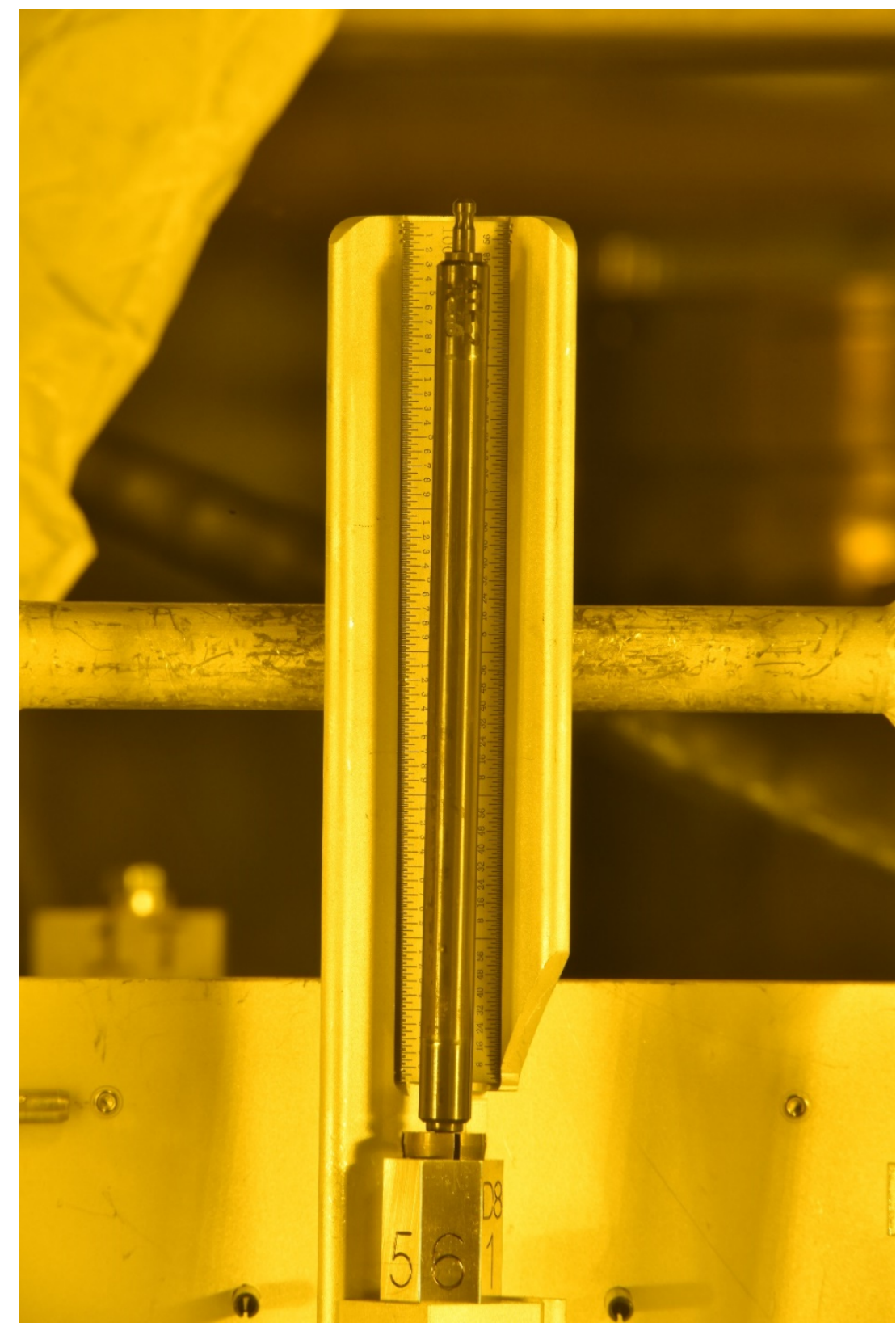

(f)

Figure 3. (a)-(f) Visual examinations of R06. 


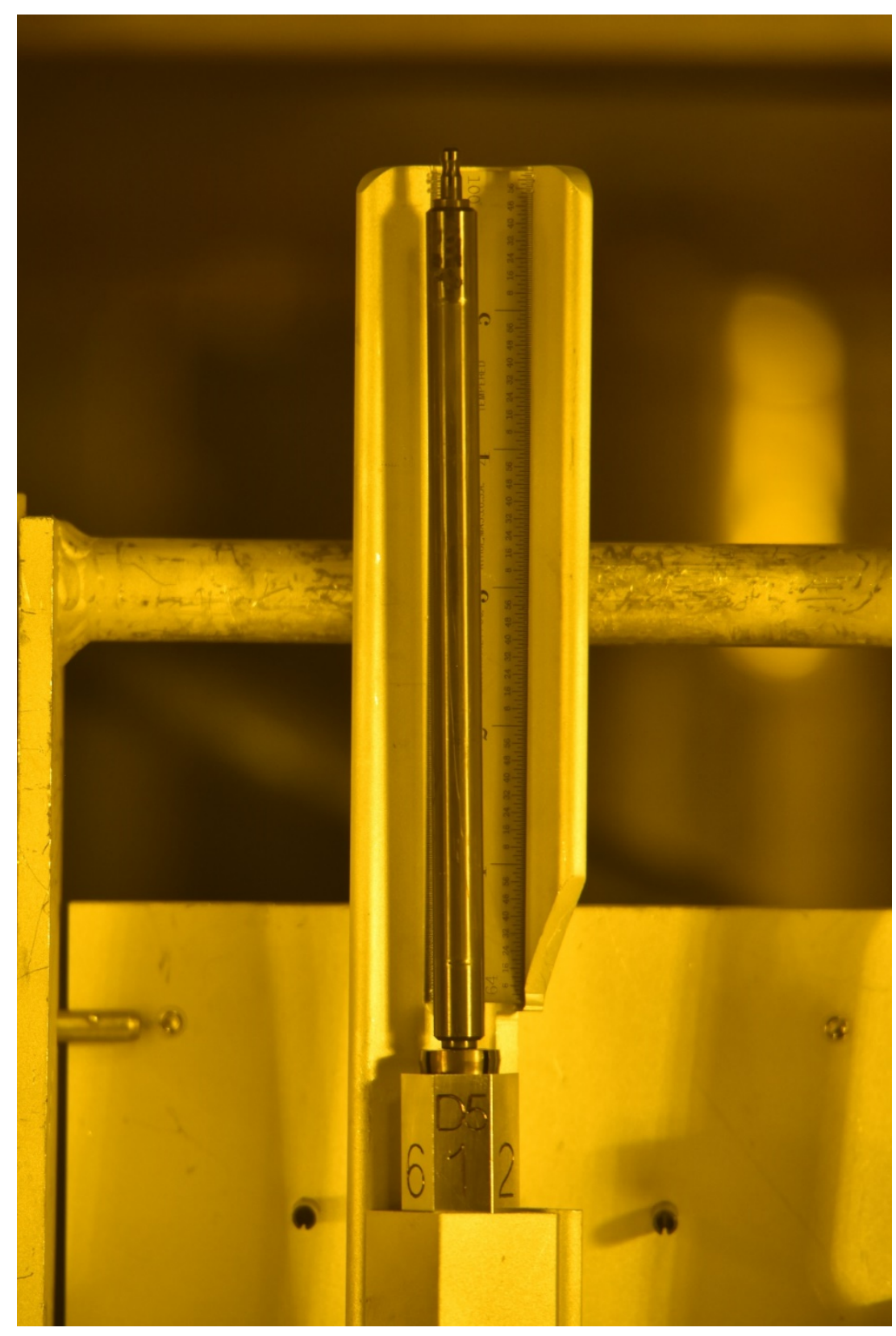

(a) 


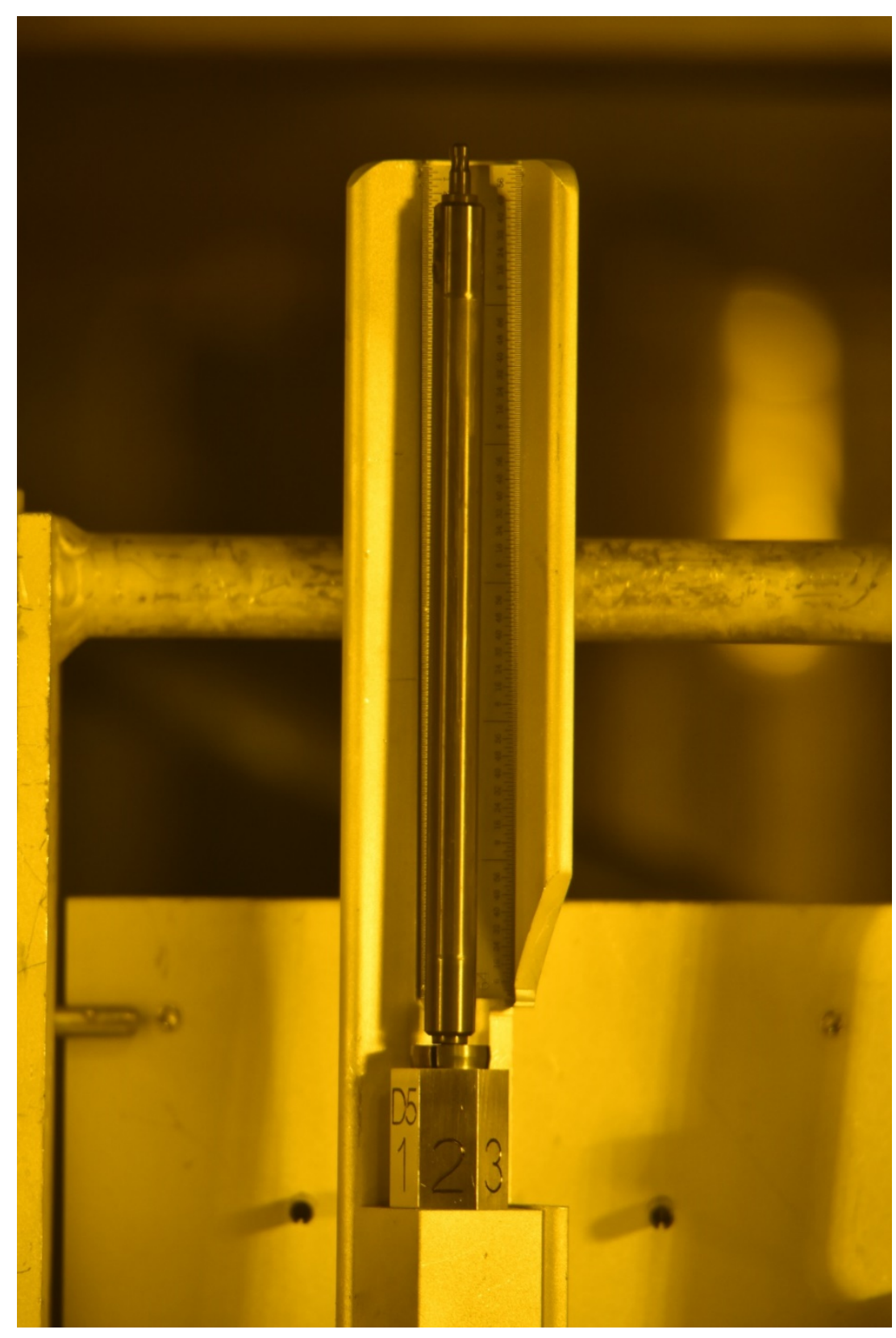

(b) 


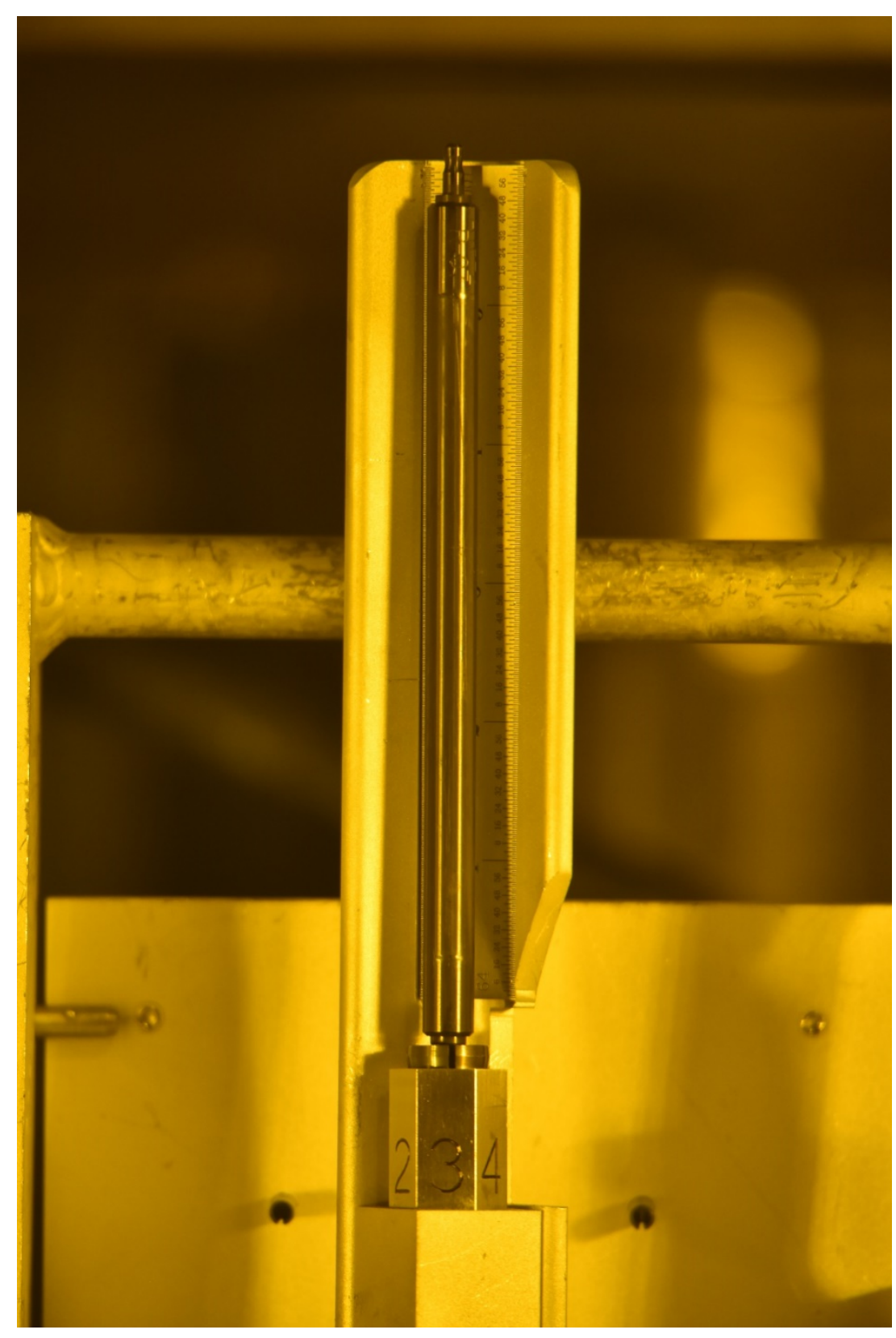

(c) 


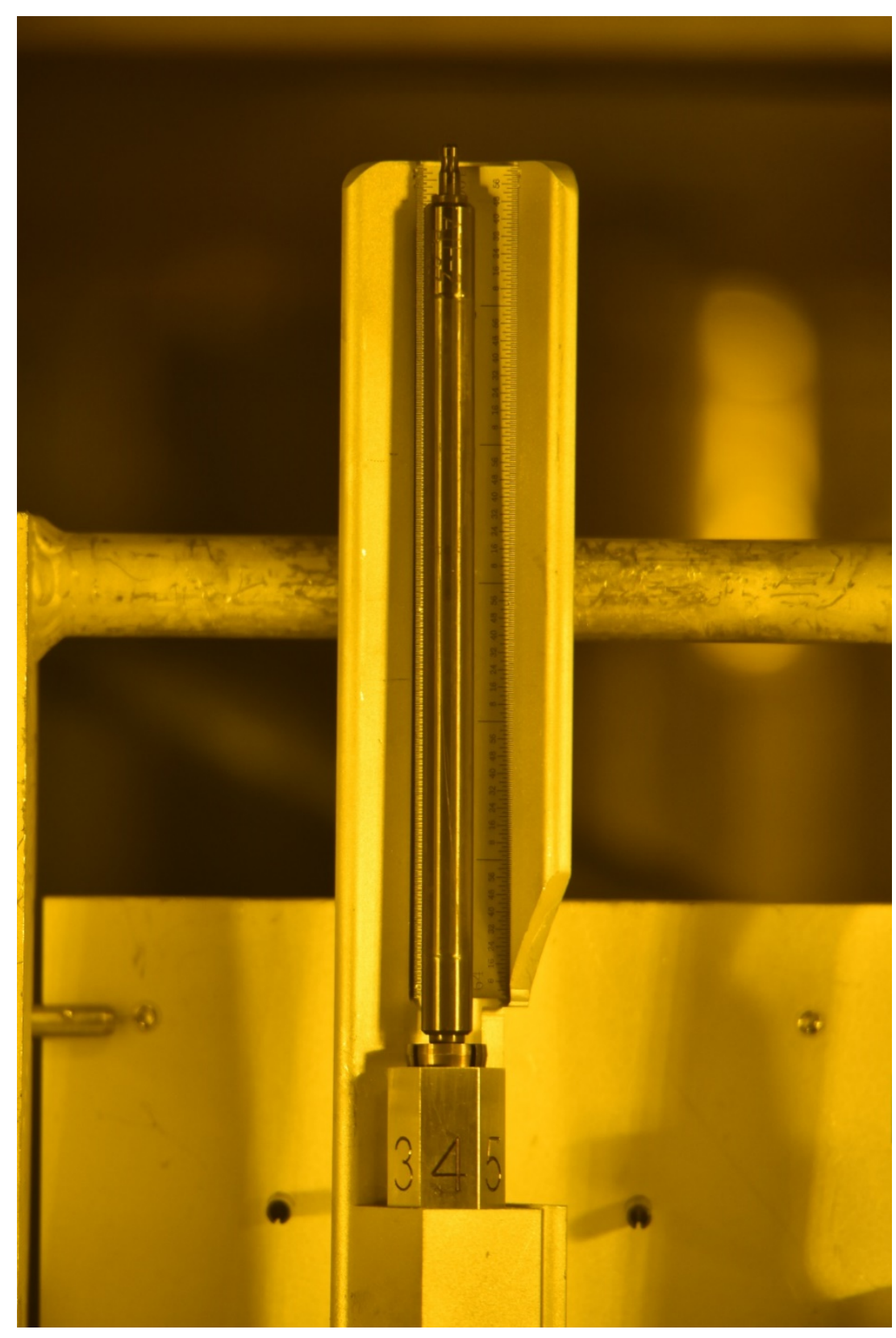

(d) 


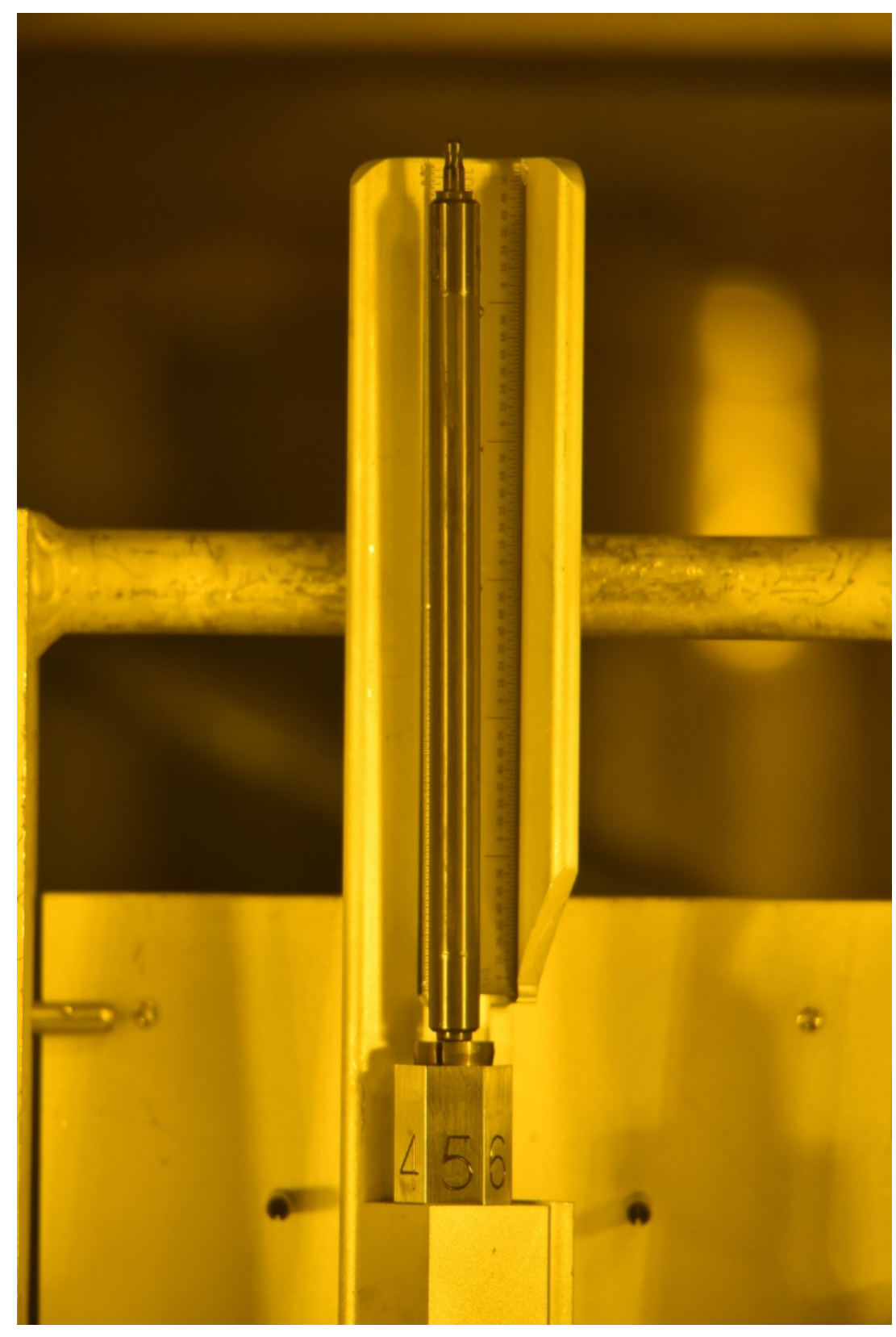

(e) 


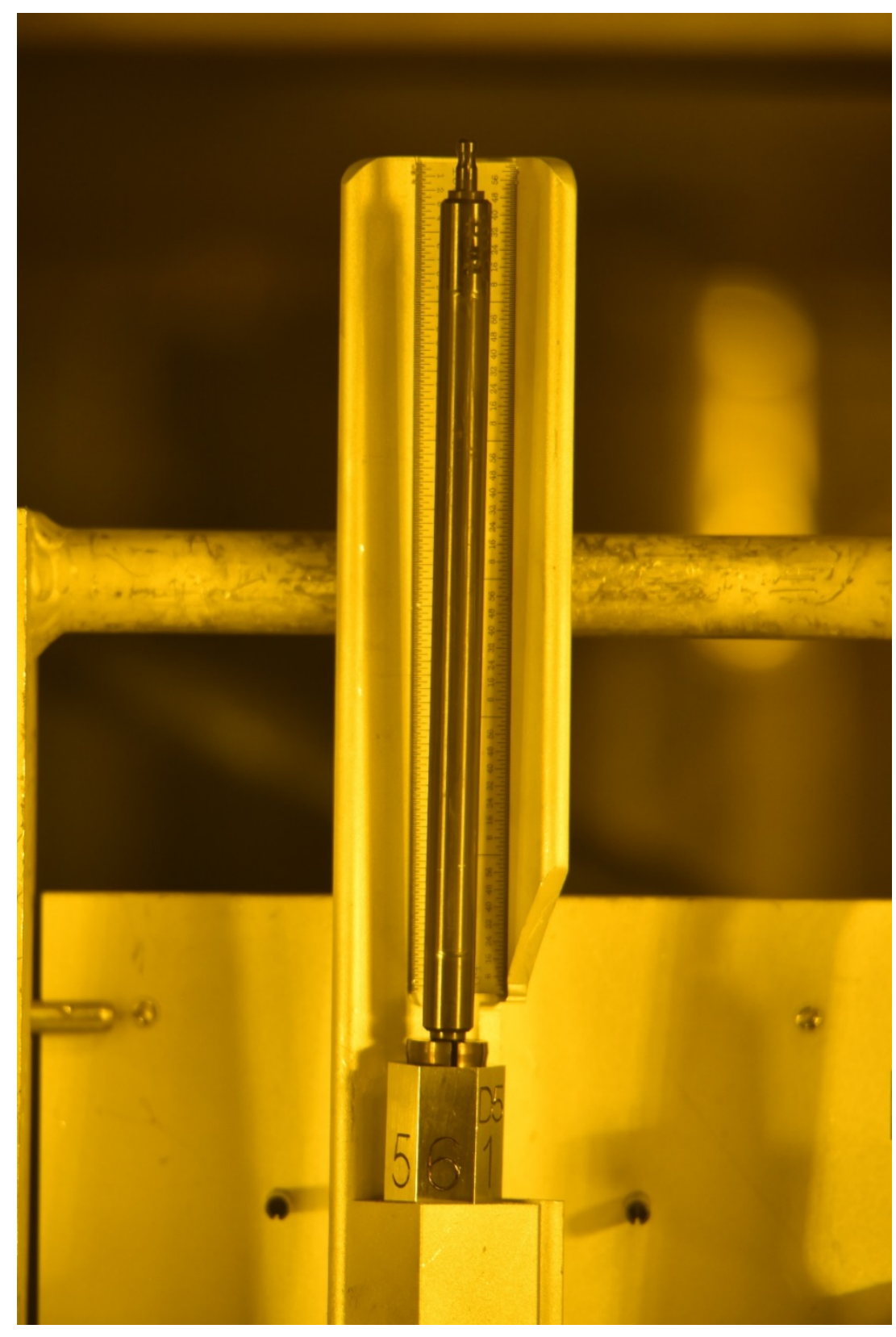

(f)

Figure 4. (a)-(f) Visual examinations of R07. 


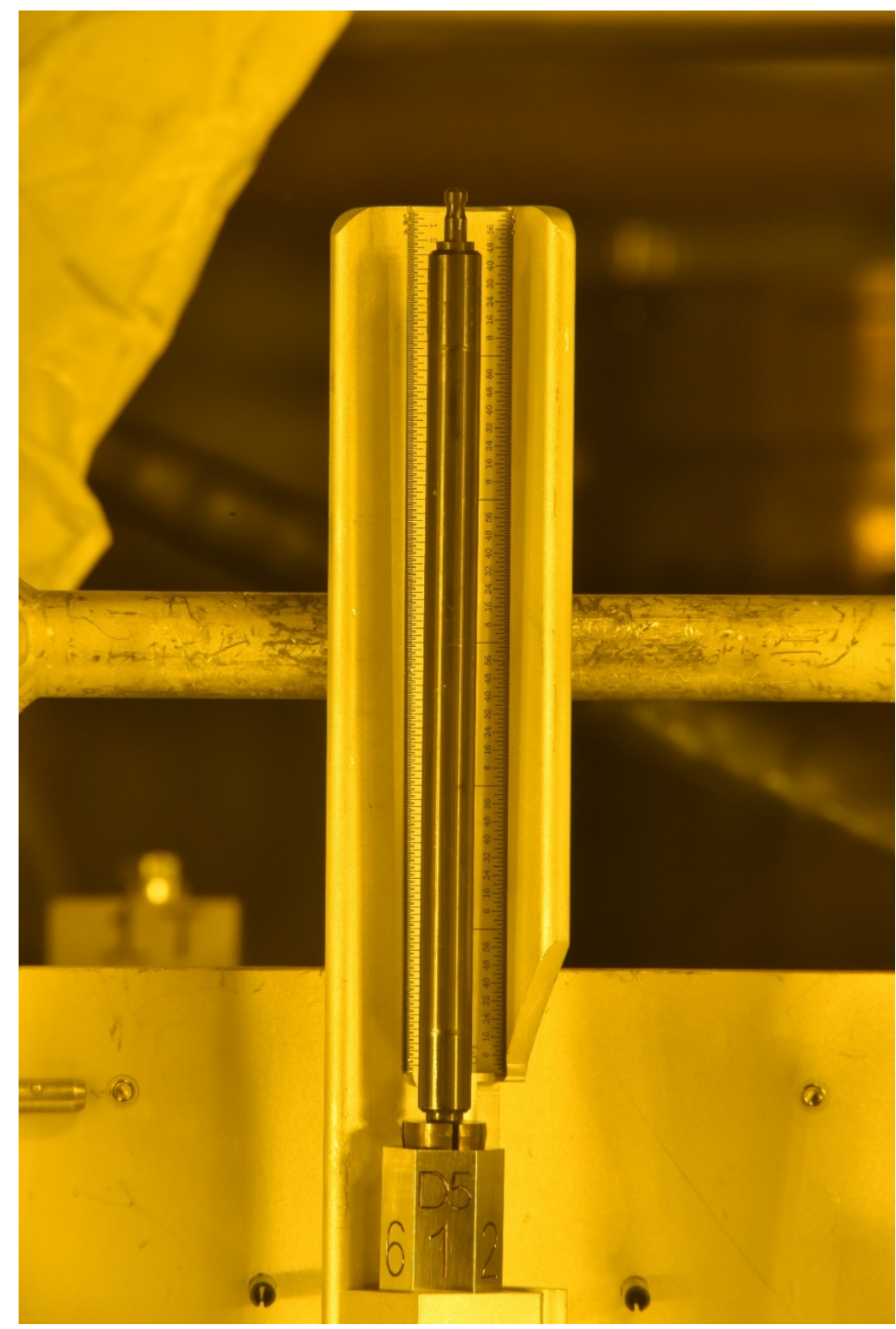

(a) 


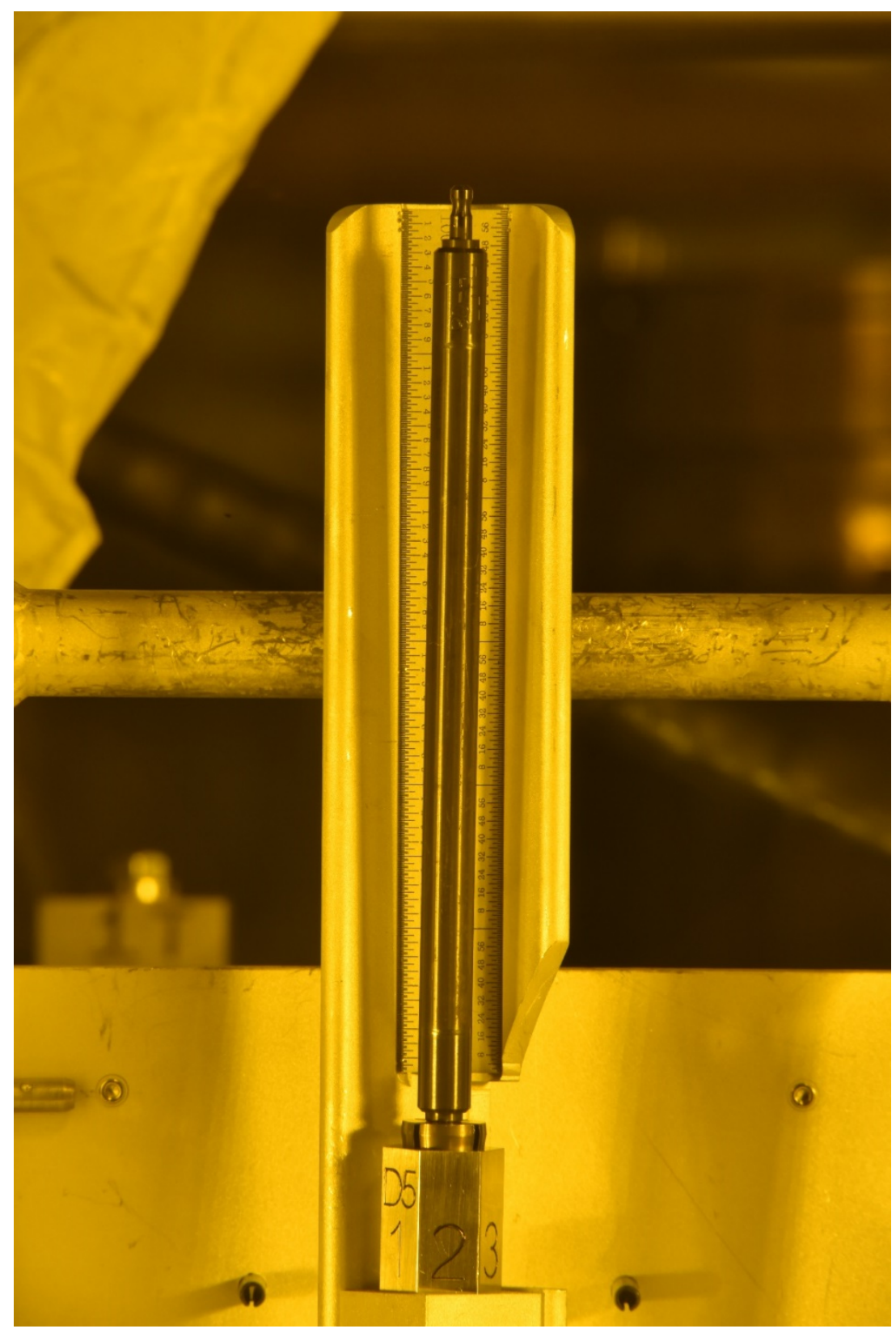

(b) 


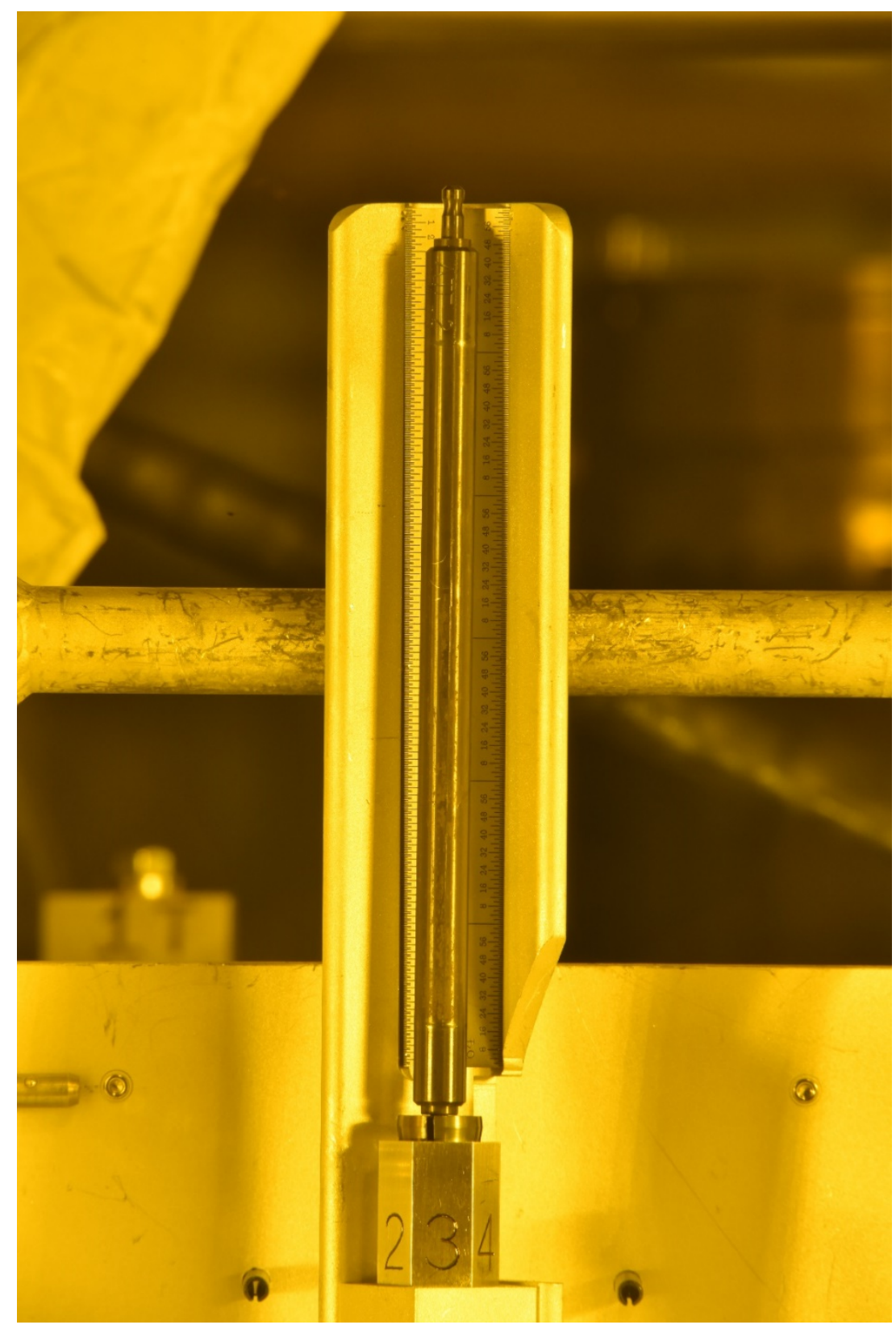

(c) 


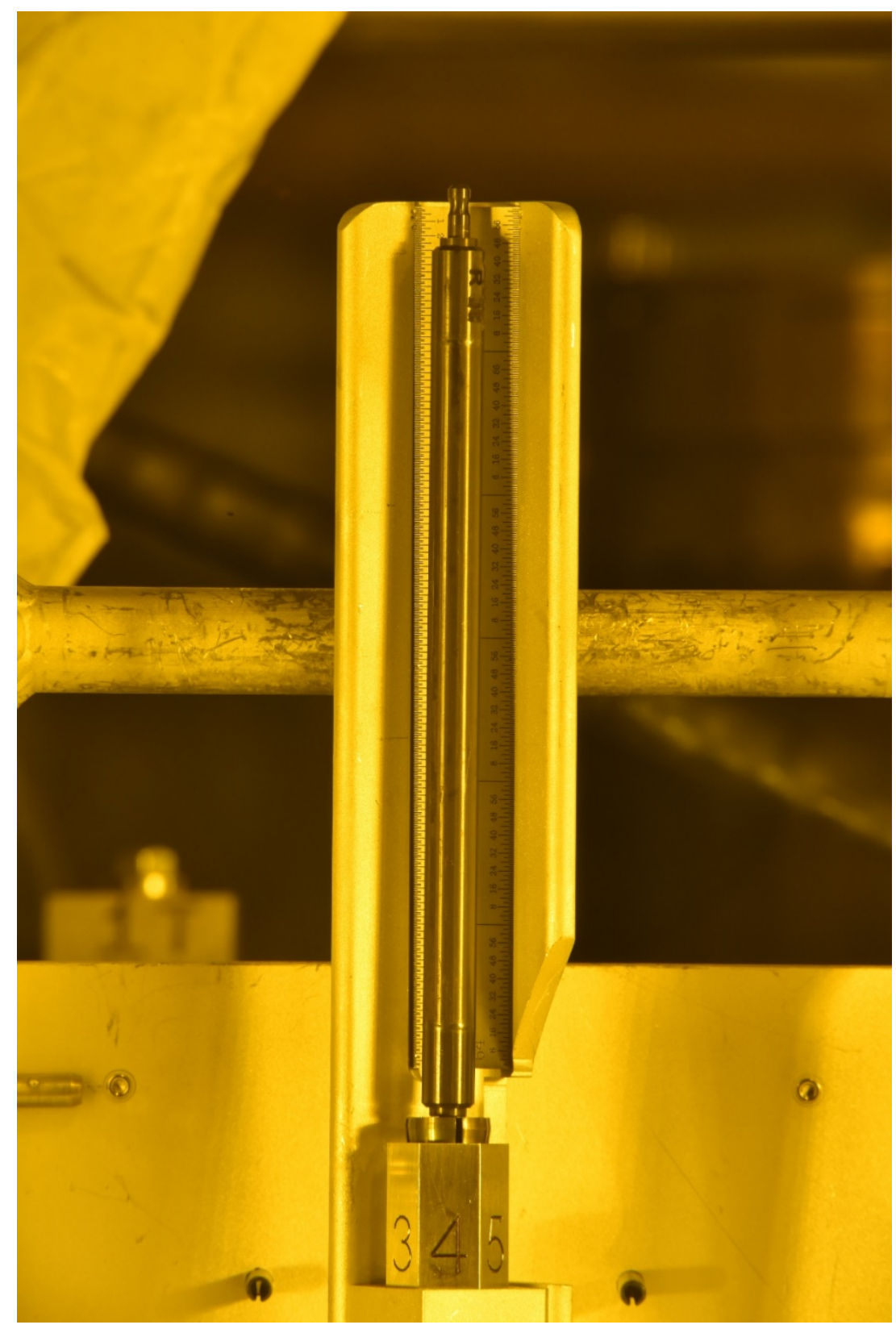

(d) 


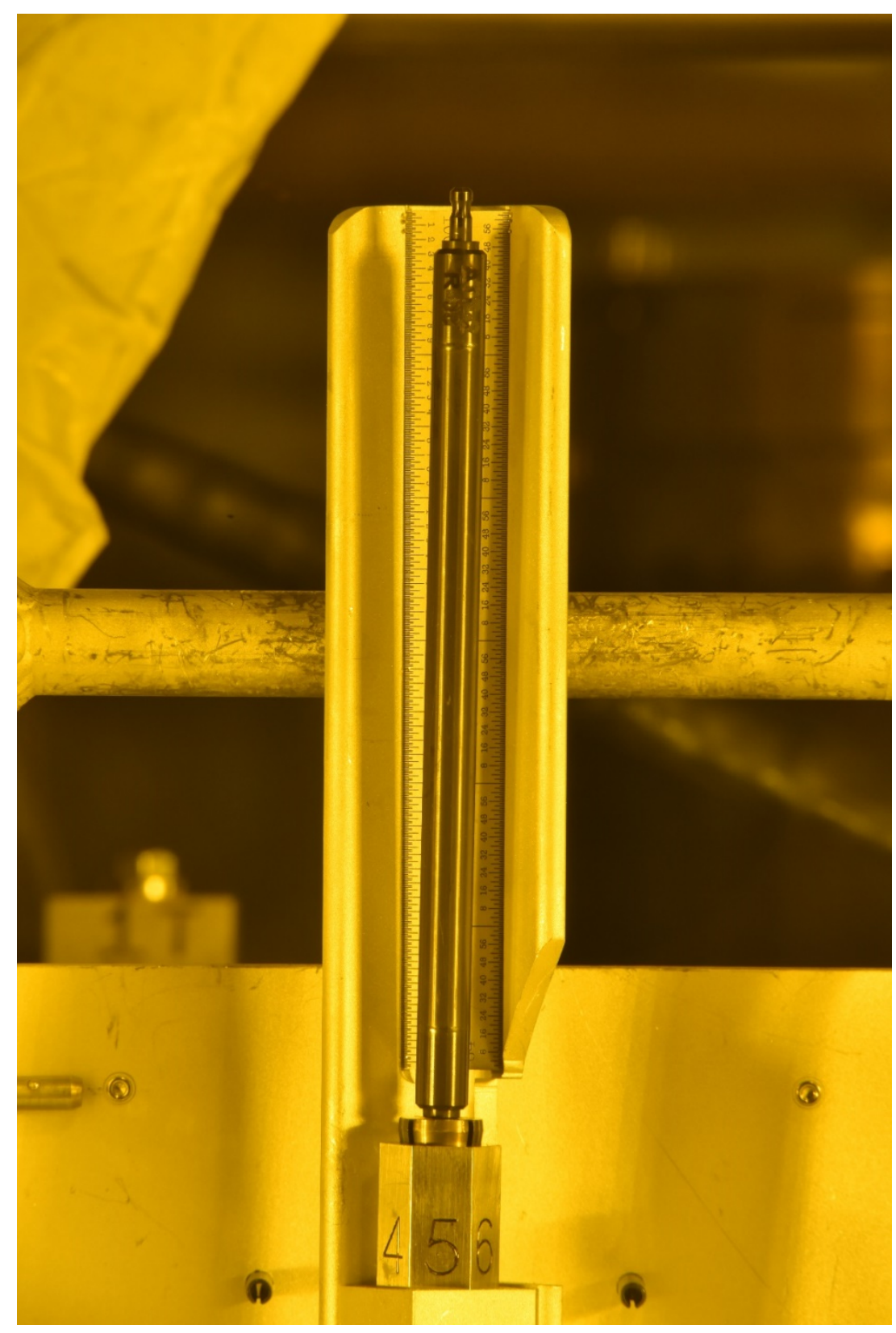

(e) 


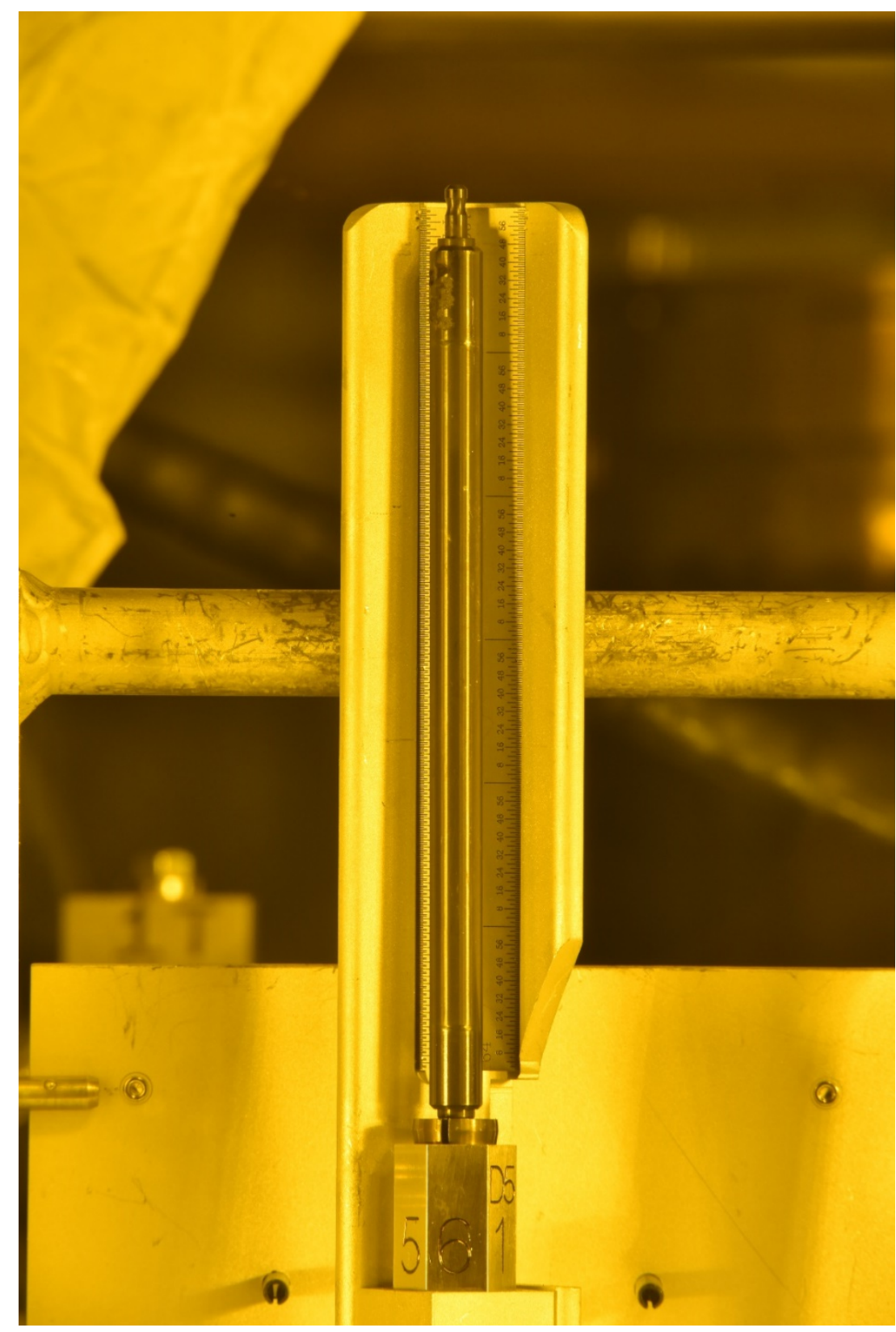

(f)

Figure 5. (a)-(f) Visual examinations of R08. 


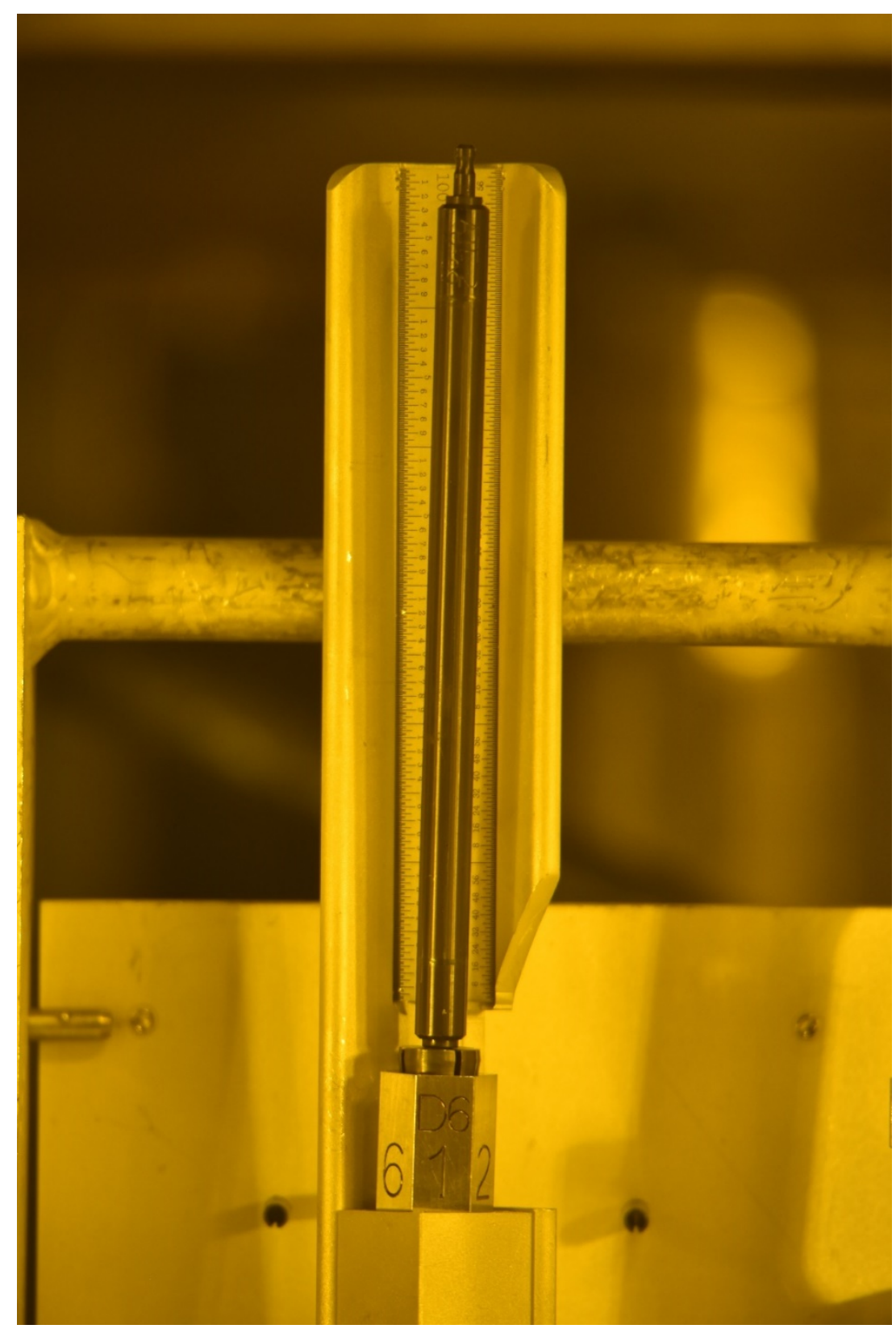

(a) 


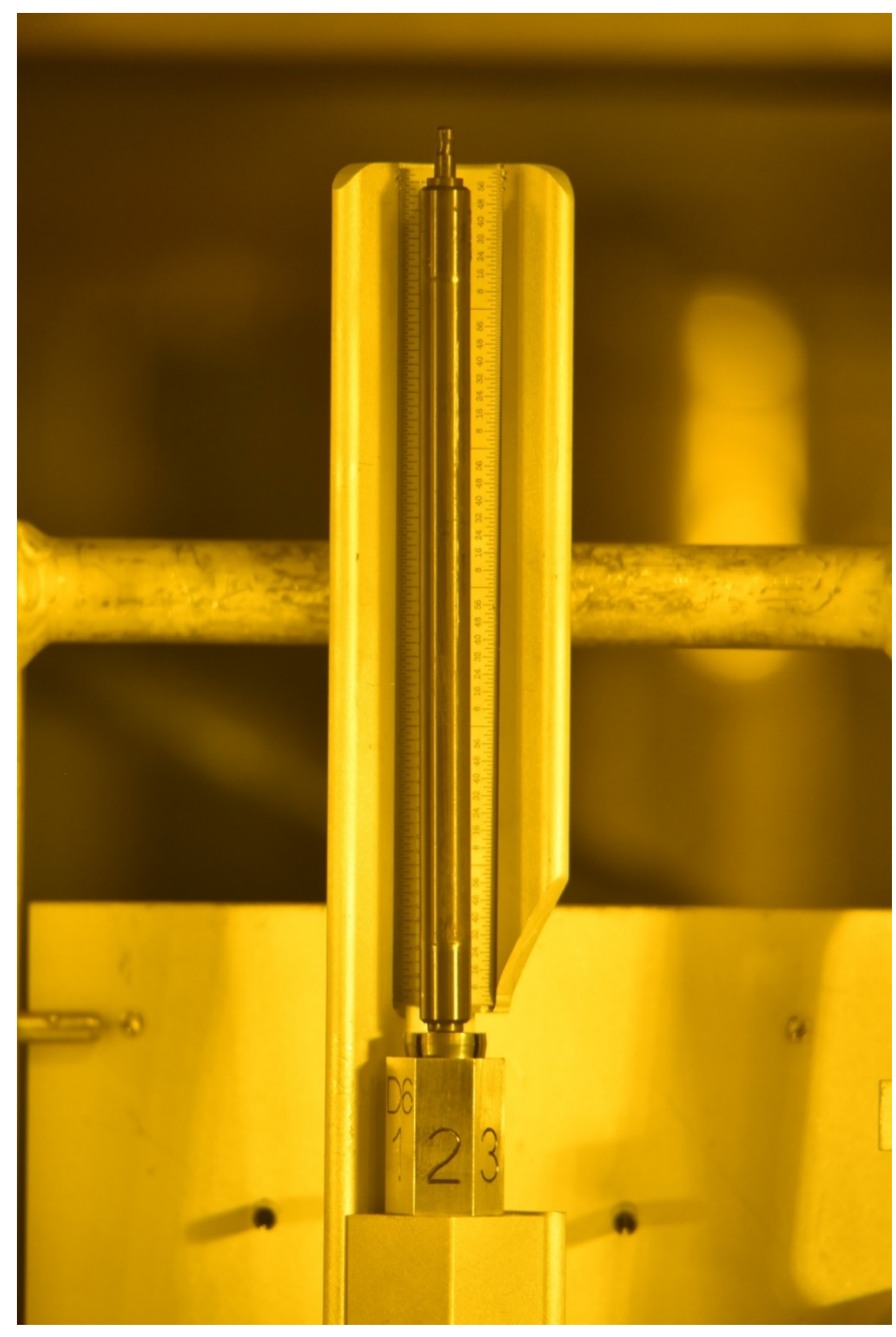

(b) 


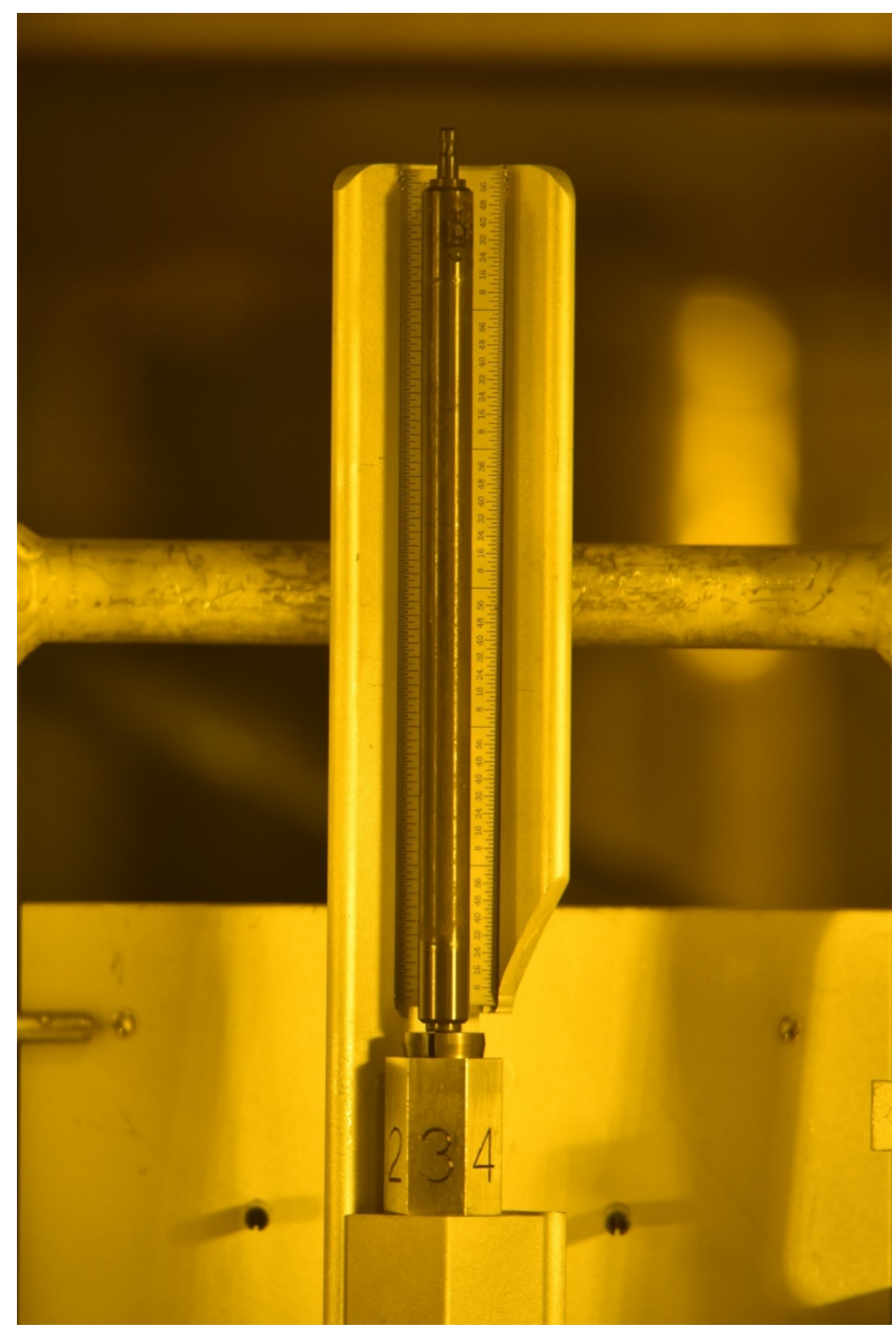

(c) 


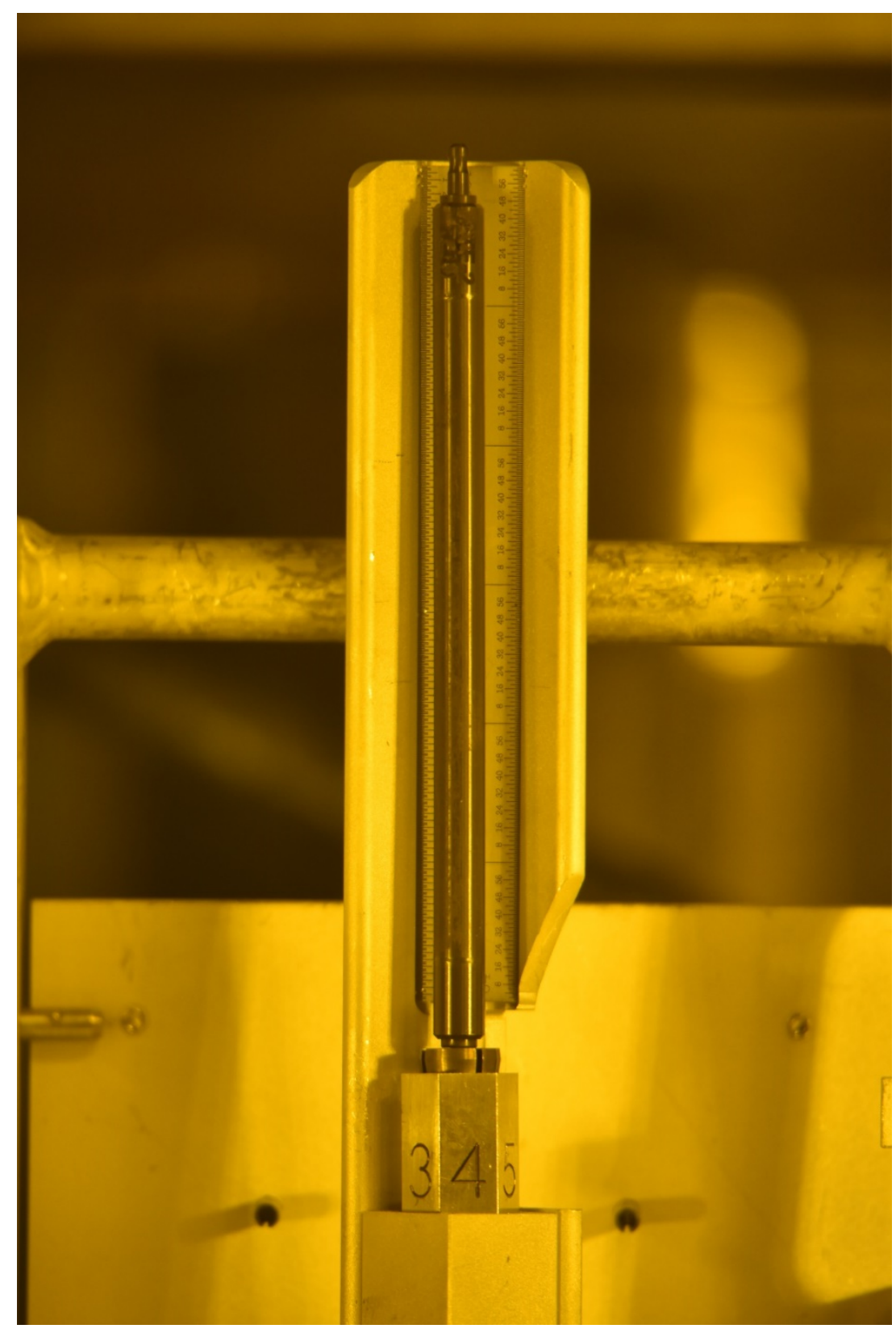

(d) 


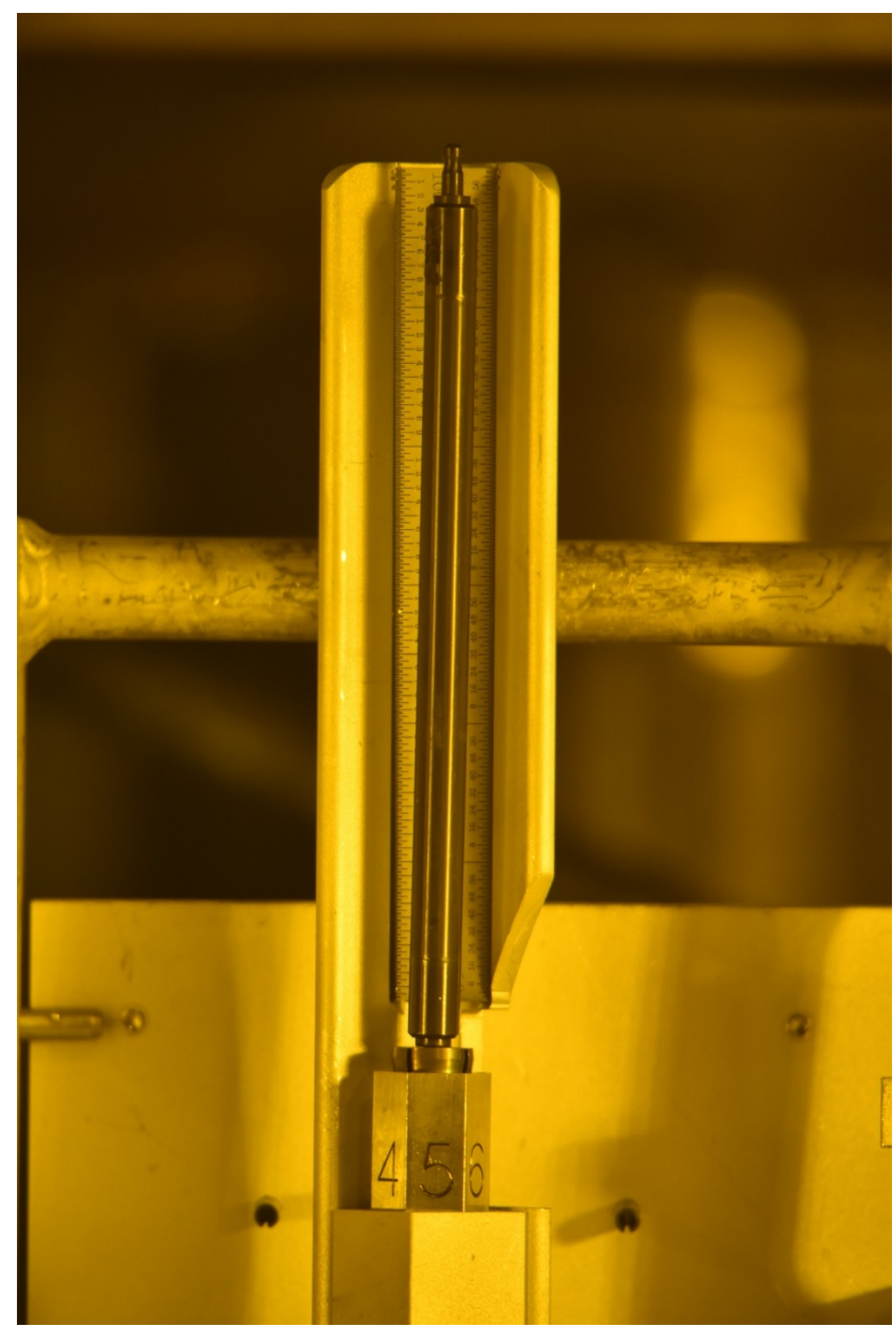

(e) 


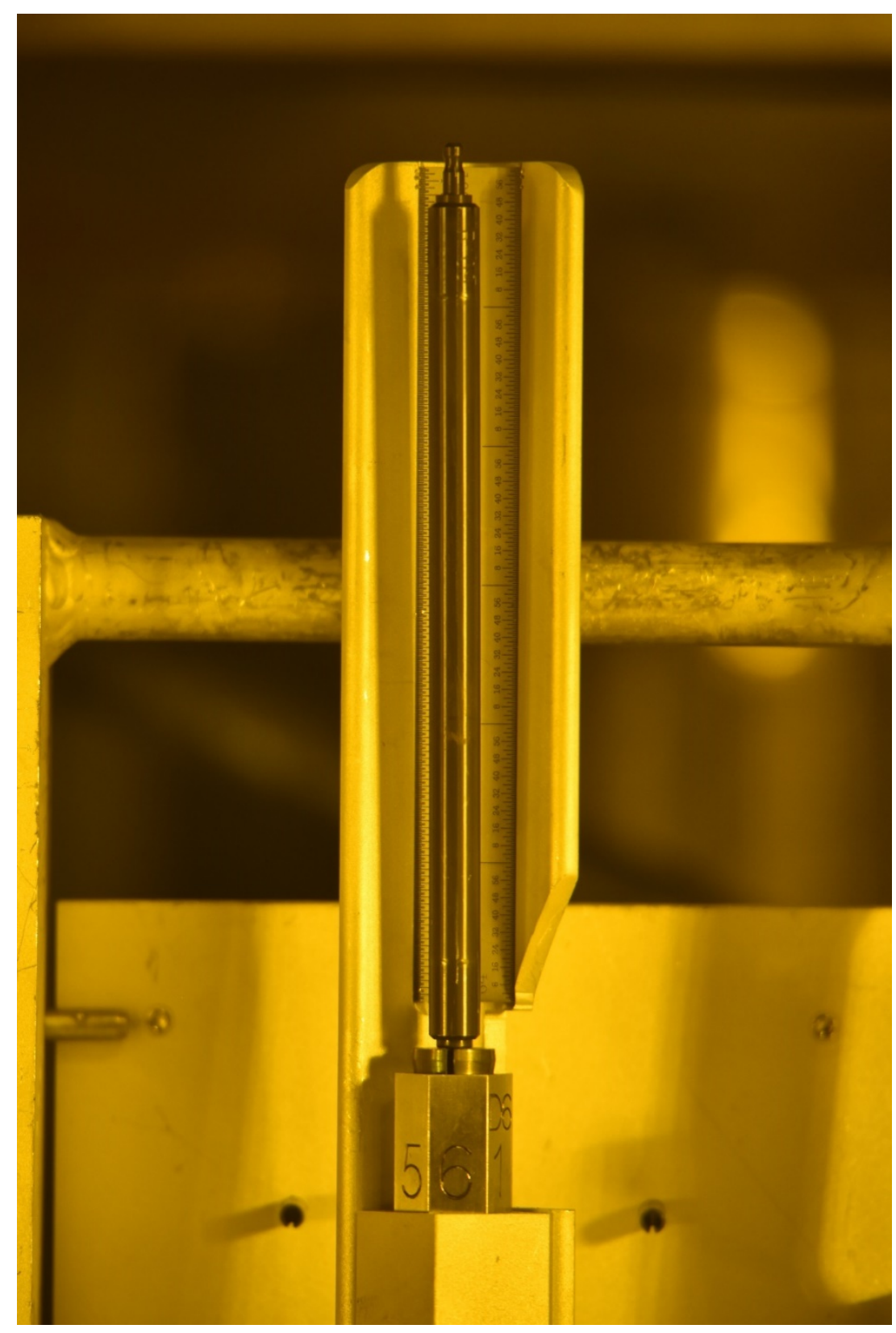

(f)

Figure 6. (a)-(f) Visual examinations of R09. 


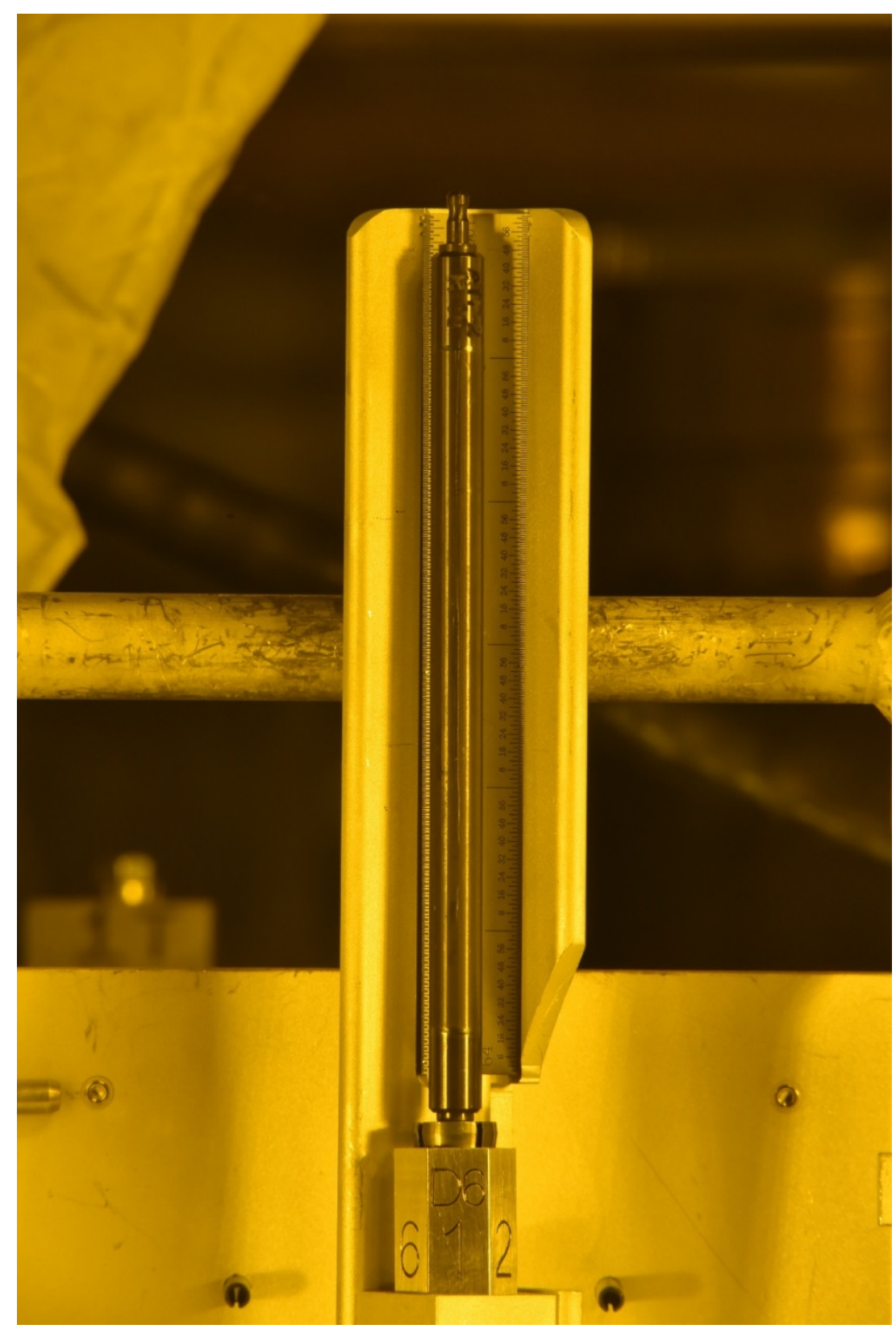

(a) 


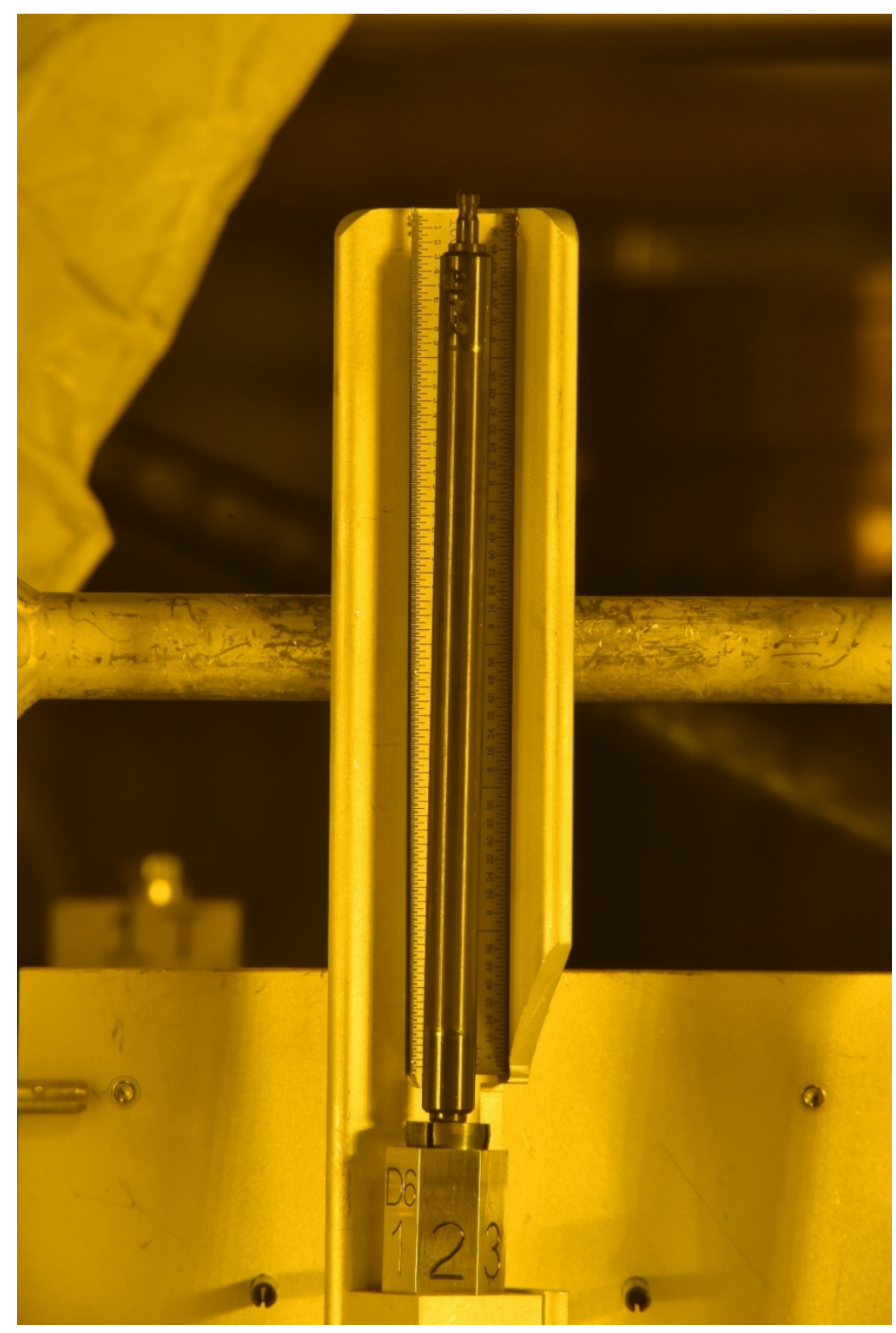

(b) 


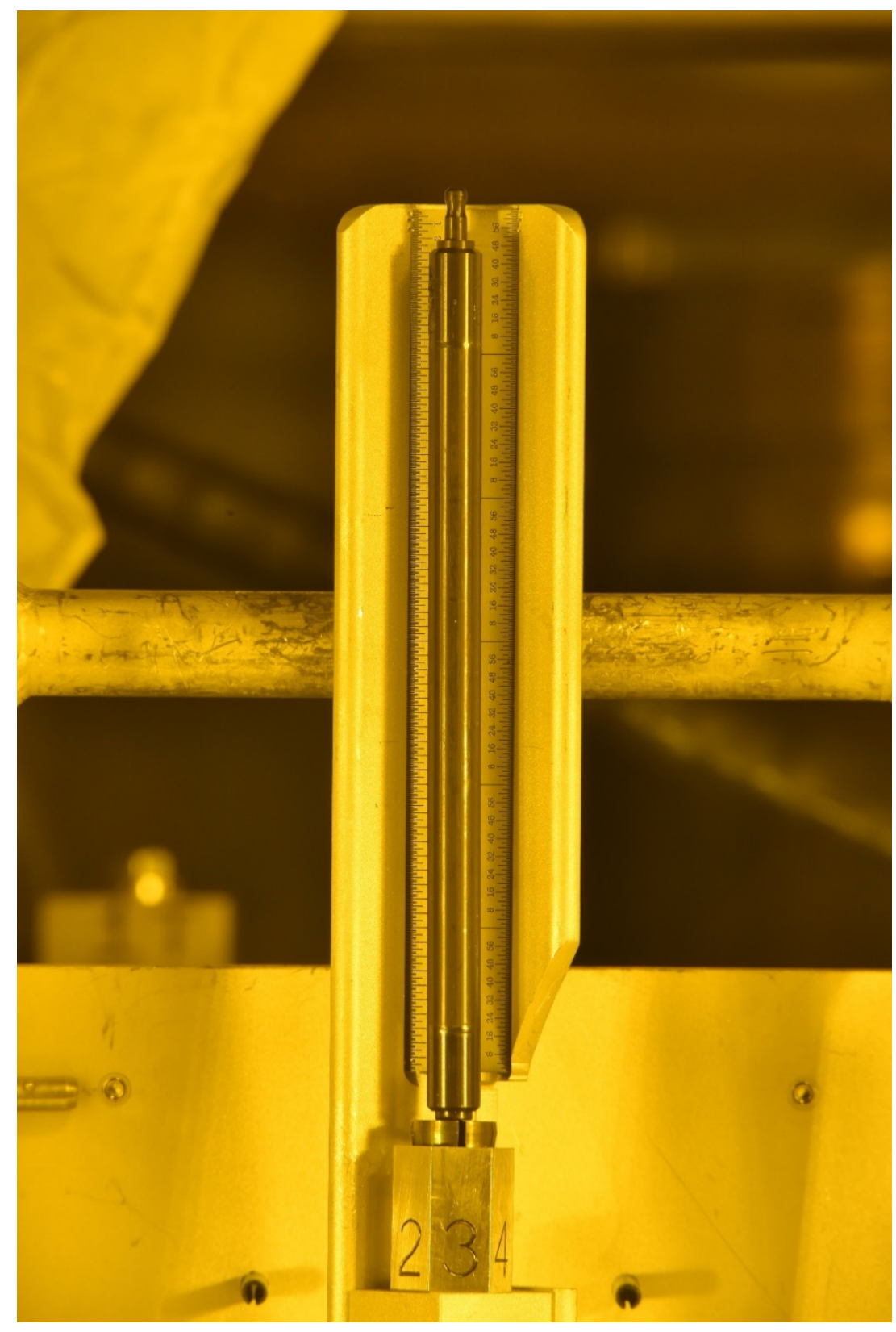

(c) 


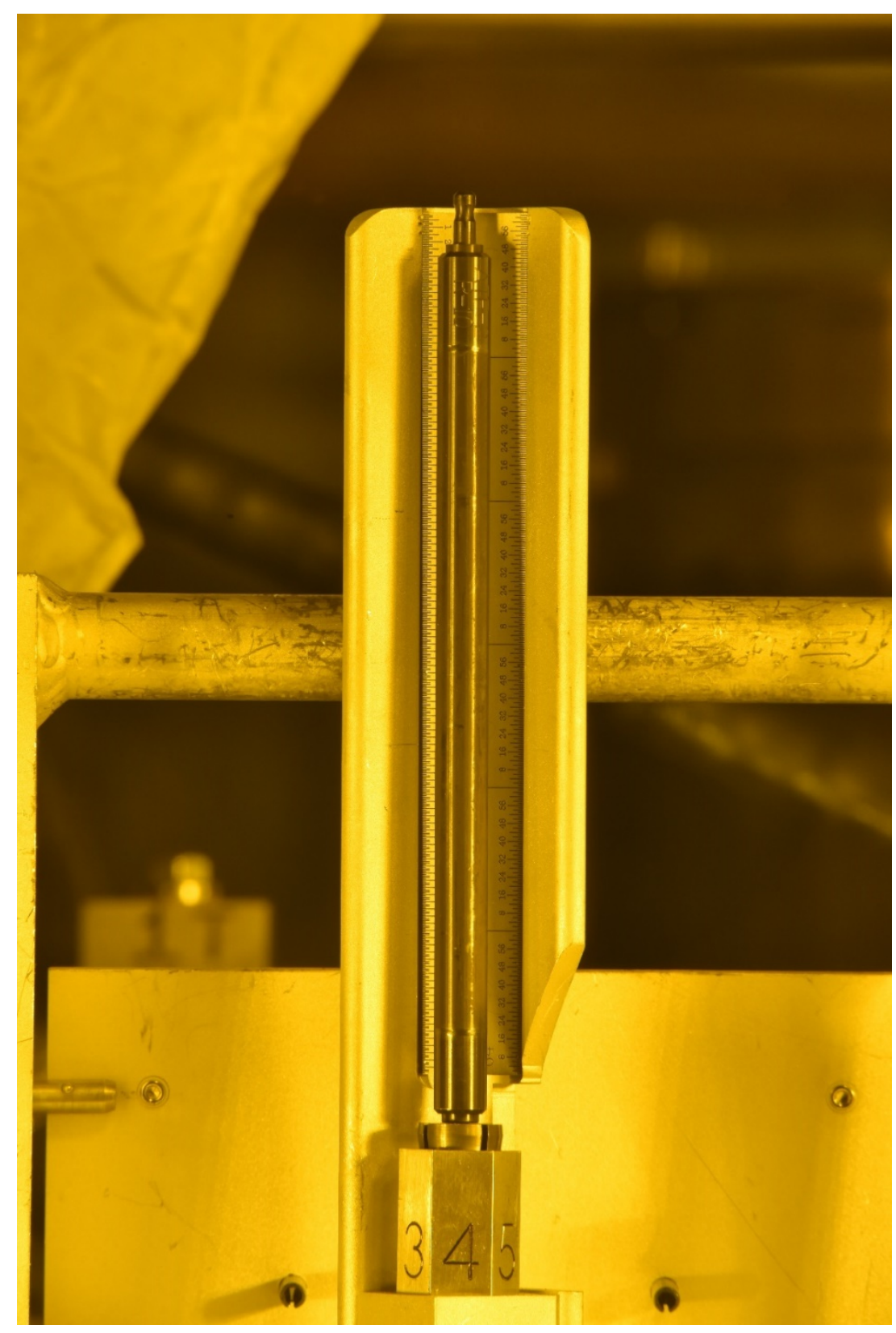

(d) 


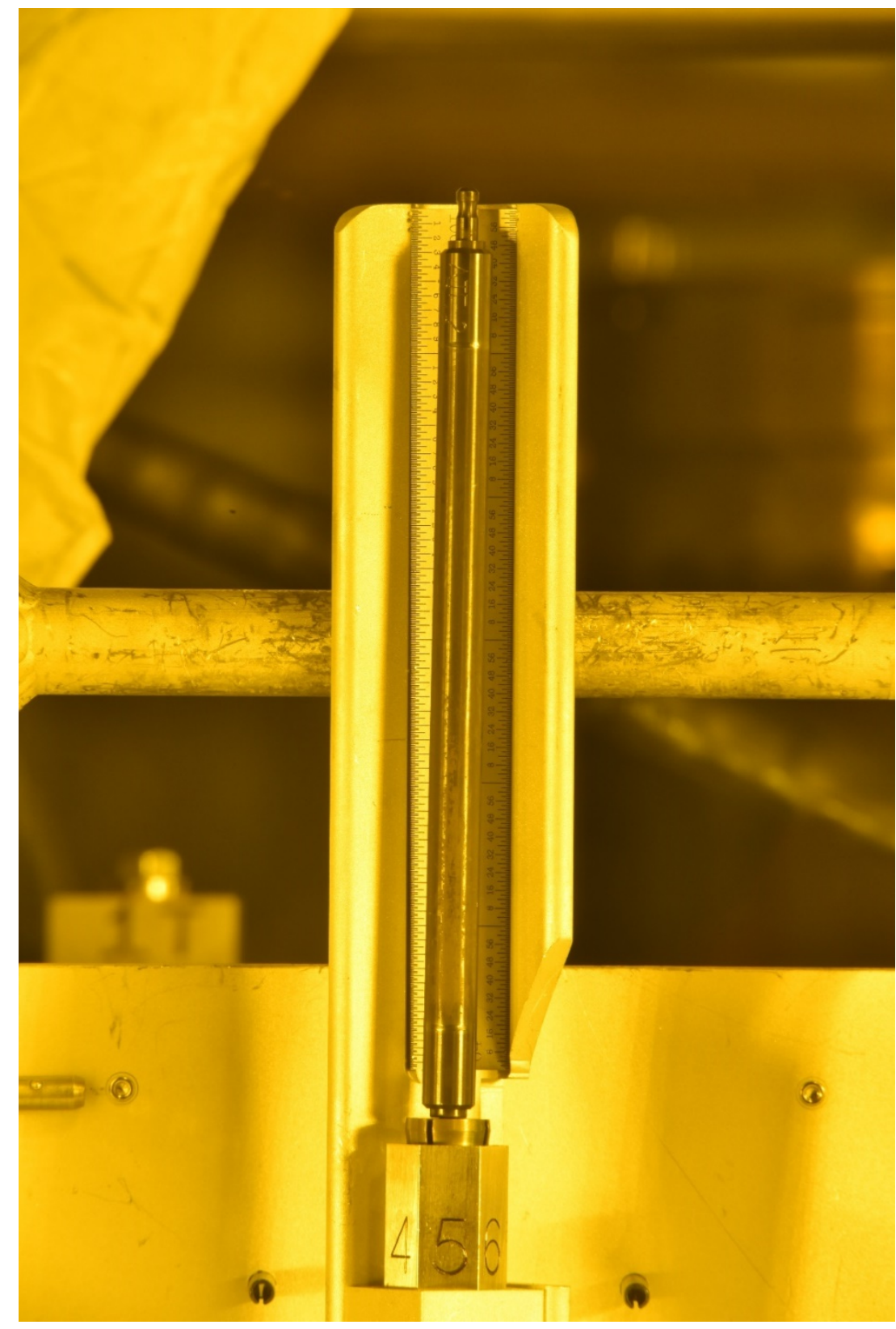

(e) 


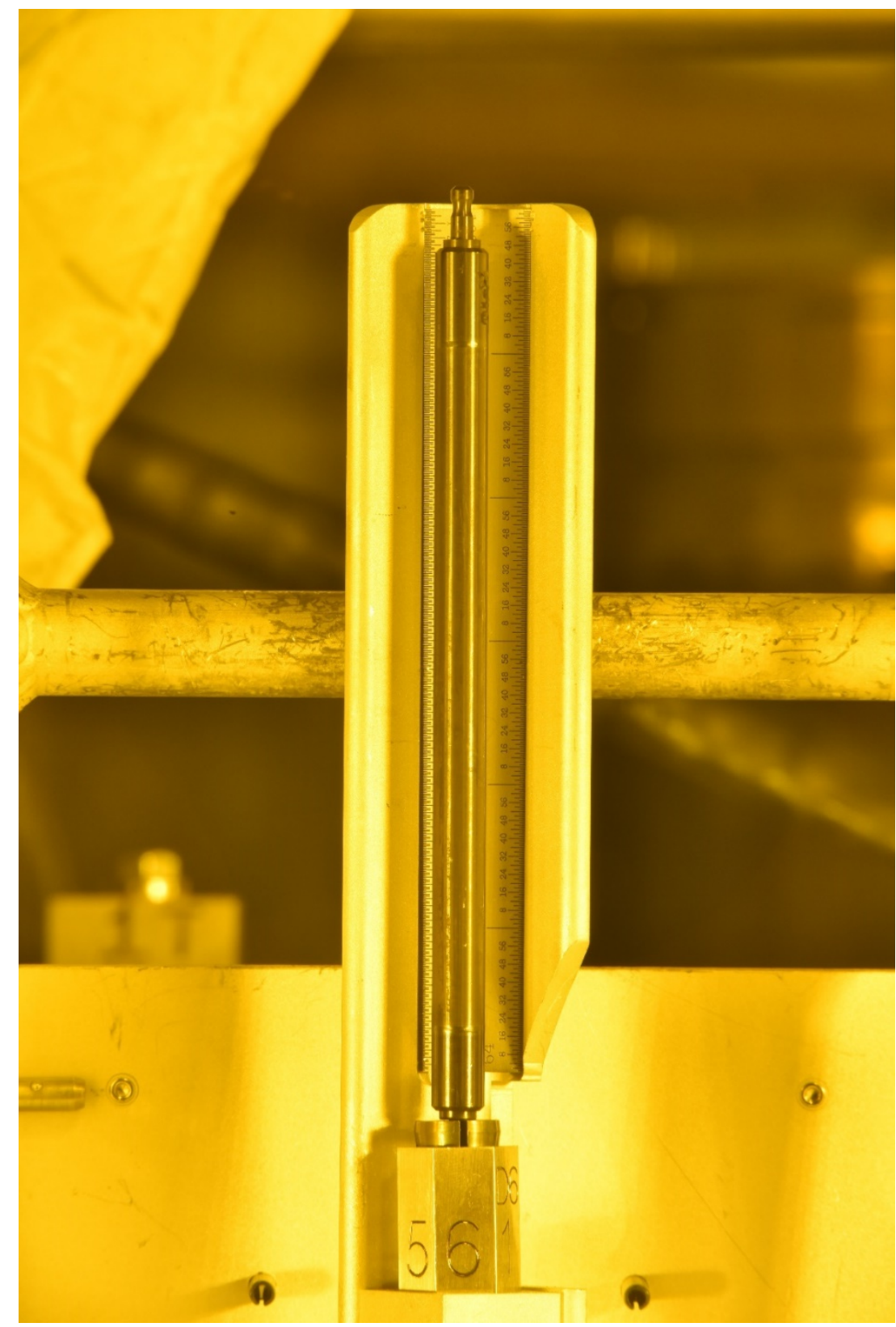

(f)

Figure 7. (a)-(f) Visual examinations of R10. 


\subsection{Gamma Spectrometry}

Gamma spectrometry of the rodlets was performed using the HFEF Precision Gamma Scanner (PGS). Details about the system have been reported in other PIE reports [3,4]. The results of some of the principal gamma emitters generally used as burnup monitors are shown in Figure 8a through Figure $8 \mathrm{~g}$ for each of the rodlets. Axial gamma spectrometry of all the rodlets showed that most detectable fission products did not redistribute axially, including the volatile Cs. An increase in the relative power towards the end of each fuel stack was expected from neutronic calculations. The pellets in the middle portion of the fuel rodlets experienced less flux due to a "shielding" effect from the top and bottom pellets, not occurring for the outermost ones. The axial depression of $\mathrm{Ru} / \mathrm{Rh}$ is larger than for the other nuclides [5]. The fission yield of metallic fission products is much larger for Pu-239 than U-235. The enrichment of $\mathrm{Pu}-239$ towards the end of the fuel stack has been predicted by the neutronic calculations [2], leading to a larger contribution to fission from Pu-239 at the outermost parts of the active fuel stack. This, in turn, would result in a higher production of metal fission products at the stack periphery, explaining the more pronounced depression of $\mathrm{Ru} / \mathrm{Rh}$ profile compared to the other fission products. Overall, the results among pins R04 to R09 are similar and consistent. All rodlets show a decreasing burnup profile while moving from the top to the bottom of the rodlet, in agreement with the axial variation of the flux in Tier 2 , where those pins were irradiated. Tier 2 is the second of the entire test train, positioned below the core center. In Figure 8c (corresponding to the results for R06), a sharp depression in the fission product signals is visible at the top of the active fuel stack. The depression corresponds to the missing fuel piece detected in the neutron radiography (see Figure 10 in the next section). This was the only anomalous result detected.

In Figure 8g, corresponding to R-10, a fluctuation in the axial power profile is visible. This rodlet was irradiated in Tier 1, which is the first holder at the bottom of the test train [6]. Being the furthest from the core centerline, an axial gradient in the neutron flux would be expected. In order to limit the variation, a hafnium shroud adjacent to only the top half of the fuel rodlets was added to the tier. The half-length hafnium shroud significantly reduced the axial power variation, as evident in the measurements. 


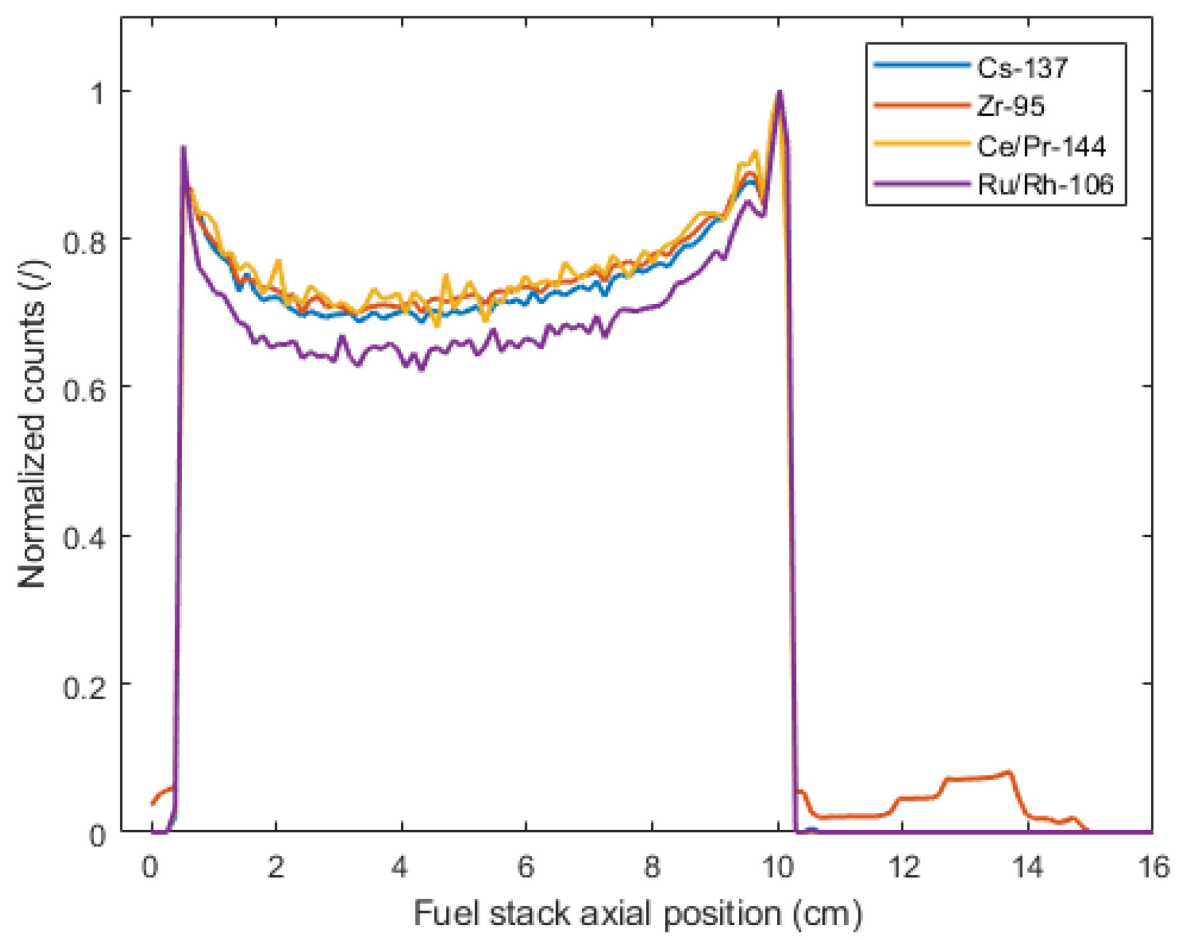

(a)

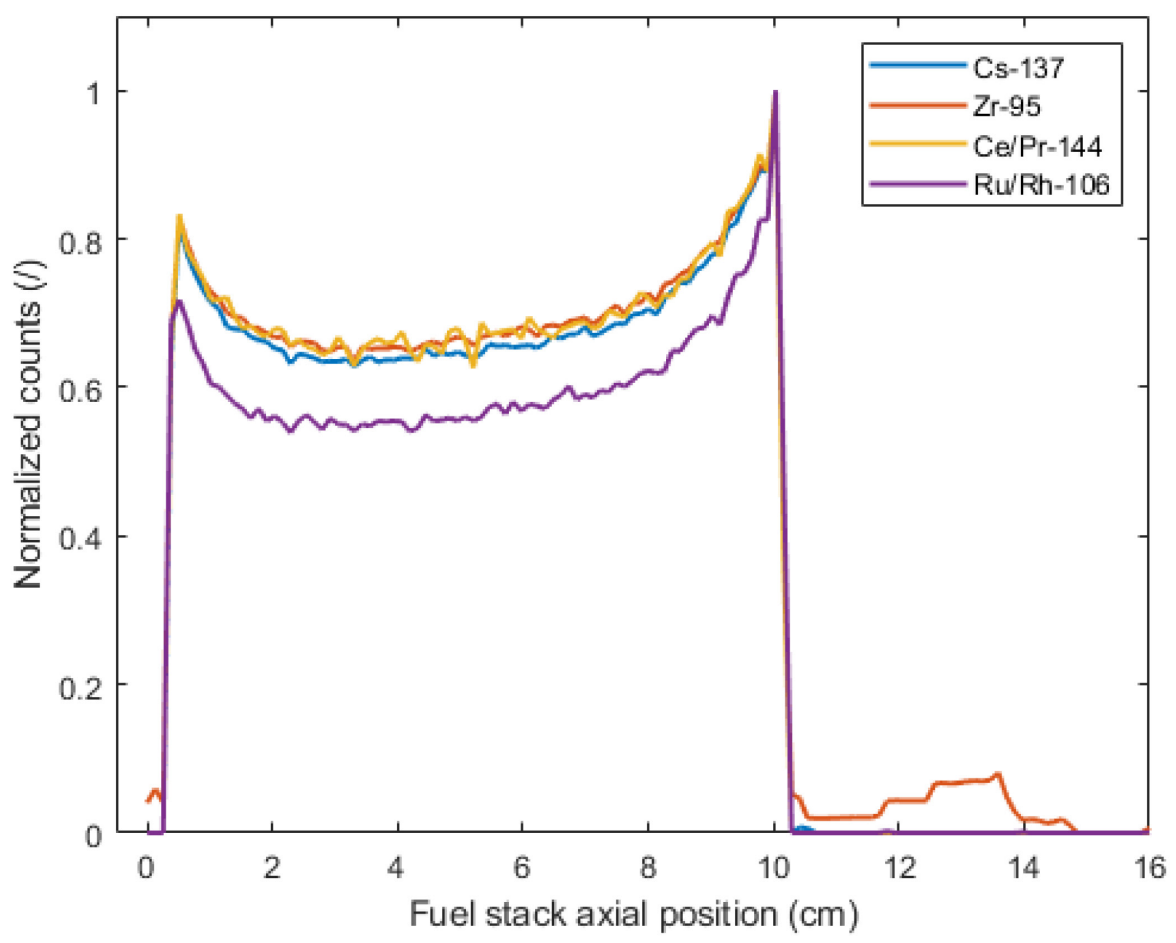

(b) 


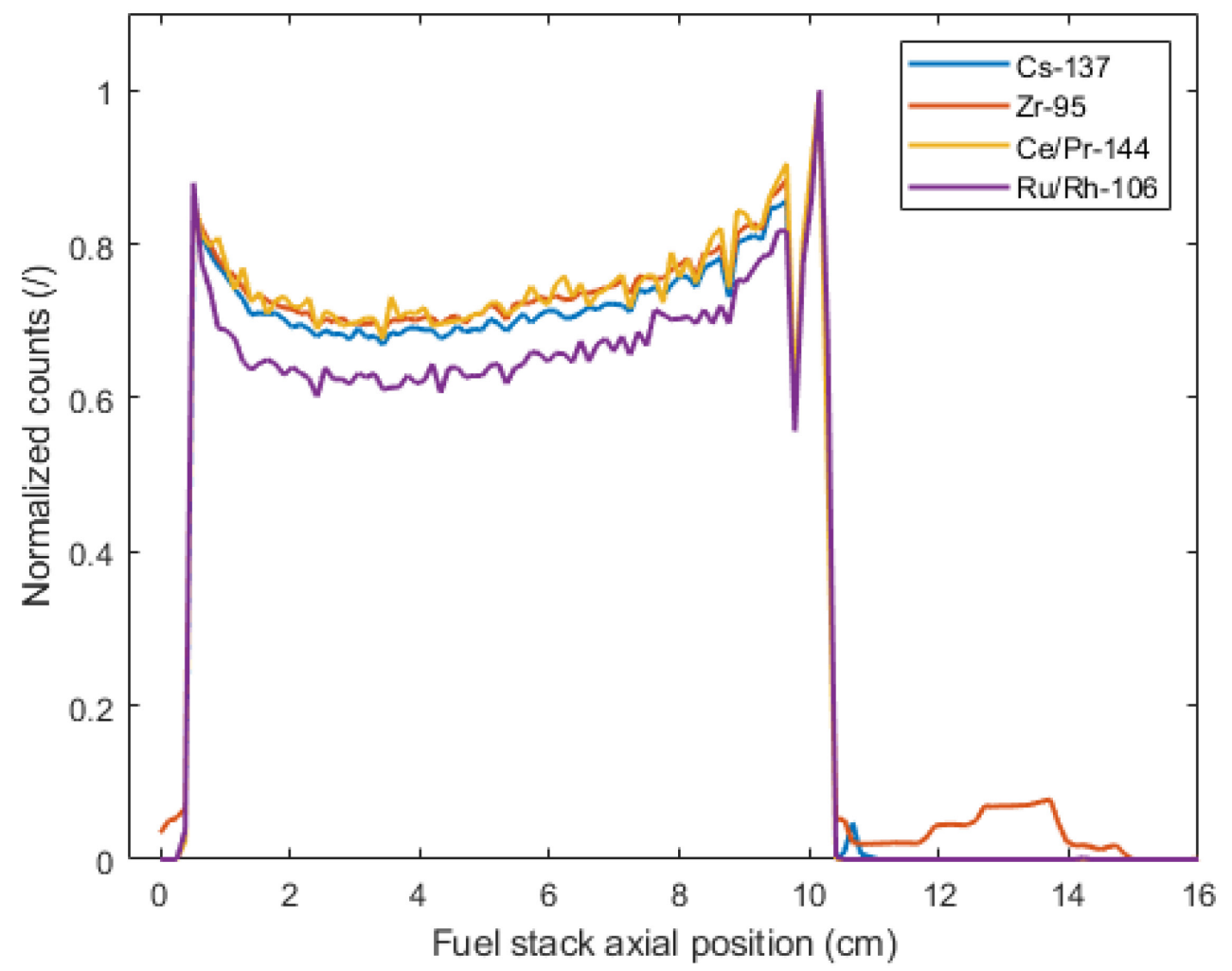

(c)

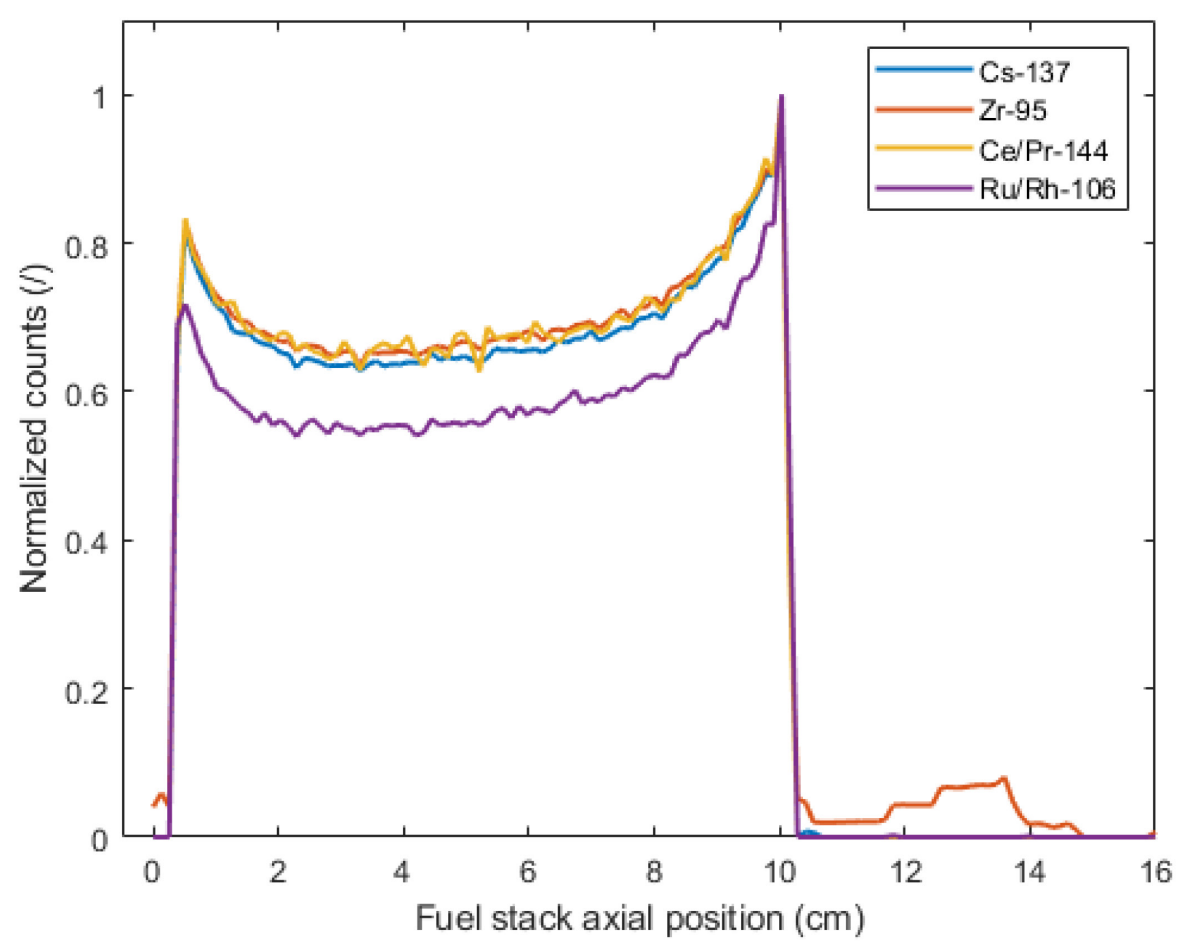

(d) 


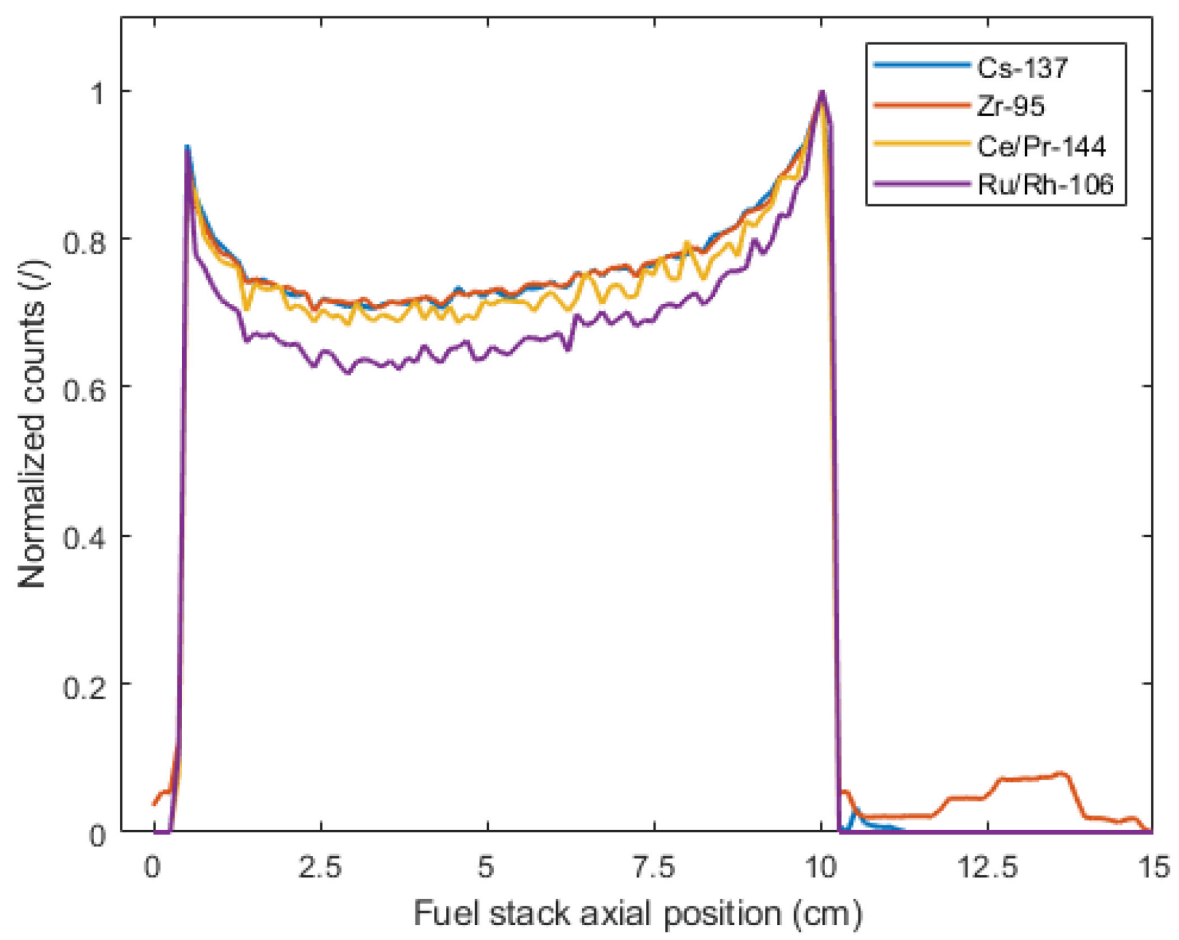

(e)

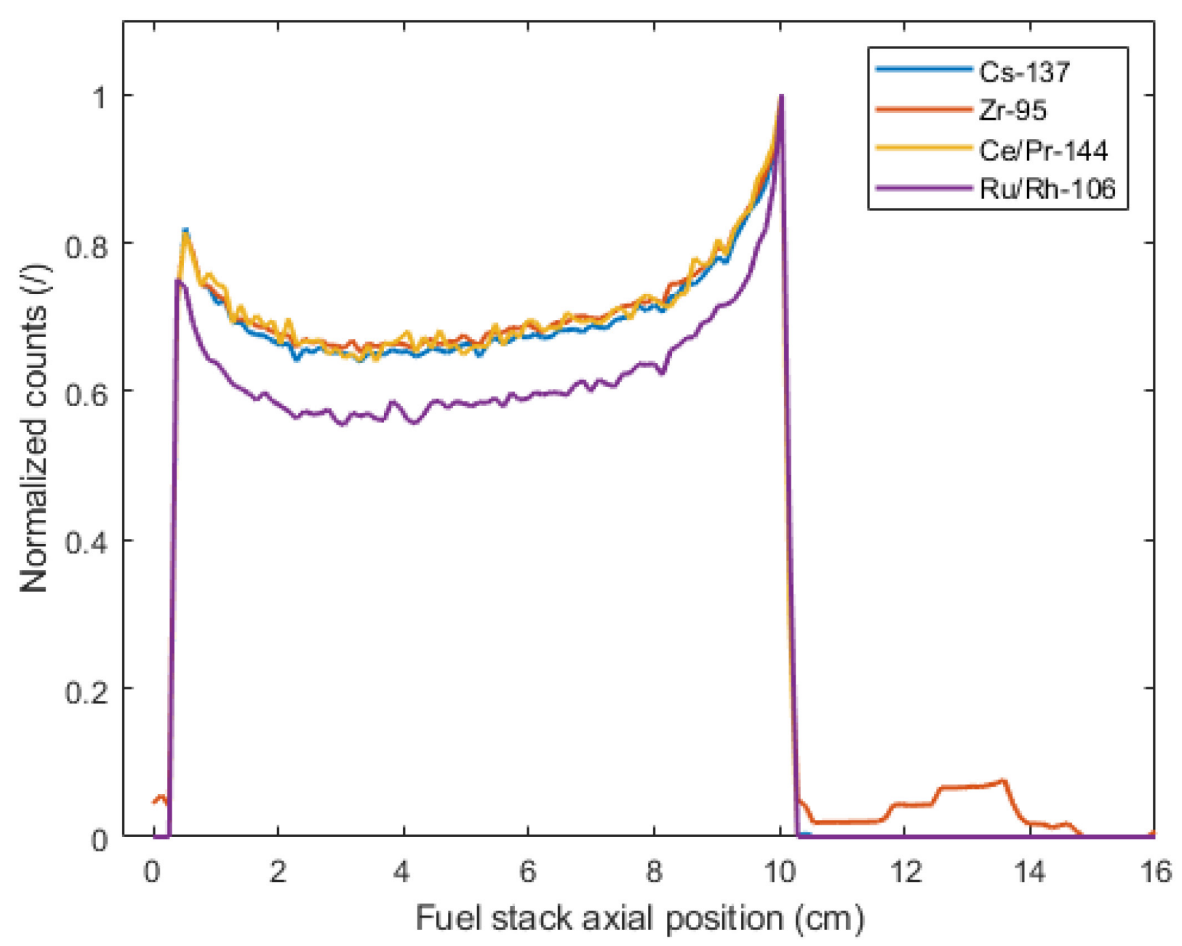

(f) 


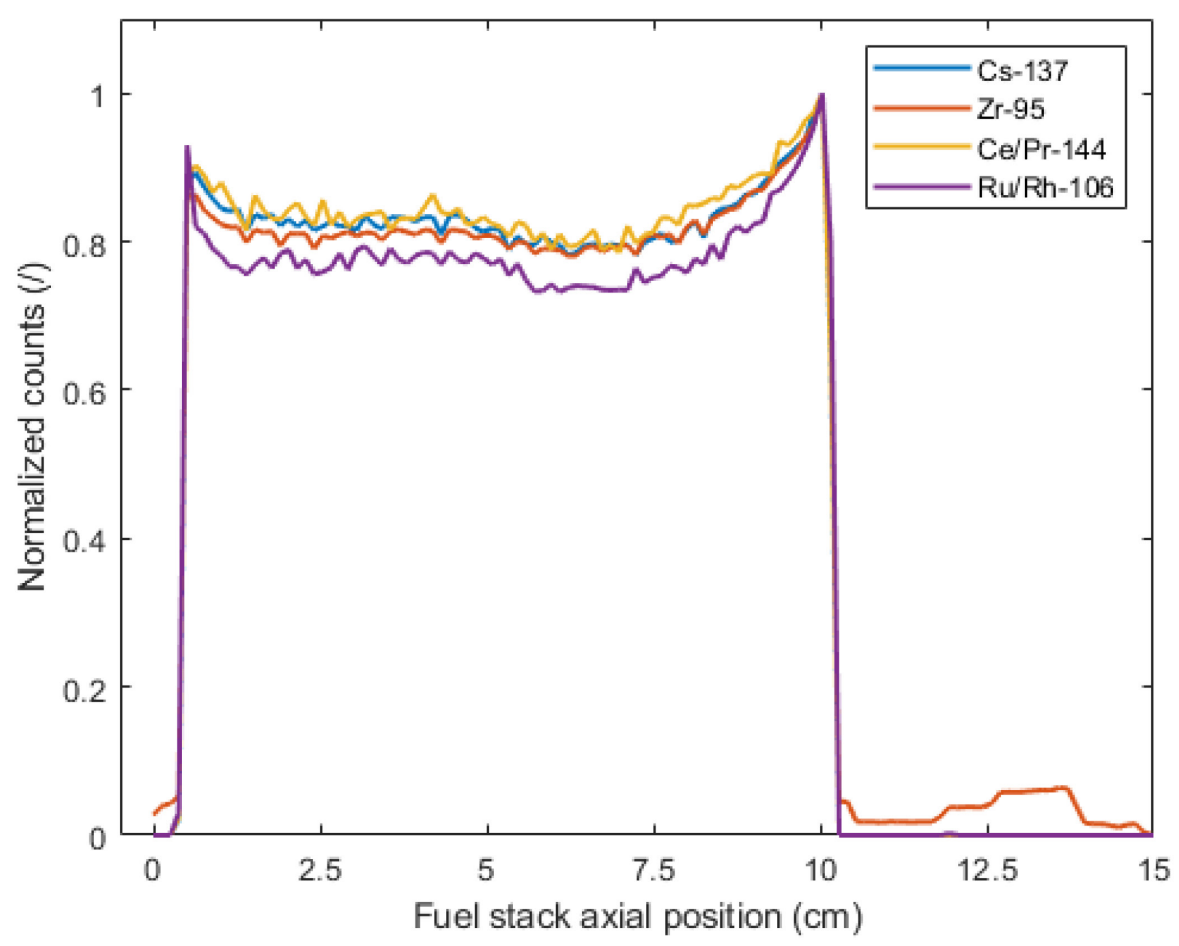

$(\mathrm{g})$

Figure 8: Axial gamma scan profile of selected fission products. The counts proceeded from the bottom of the fuel stack (x=0) till the top of each rodlet. (a) R04, (b) R05, (c) R06, (d) R07, (e) R08, (f) R09, (g) R10.

\subsection{Neutron Radiography}

Neutron radiography of the rodlets was performed with indirect radiography using the Neutron Radiography (NRAD) Reactor located in HFEF [Error! Reference source not found.]. An existing carrier could be used by adapting the height of the collets, which were designed and fabricated specifically for this PIE campaign. The collet is placed in the socket which allows for precise rodlet rotation to acquire images at different orientations. Radiographs of the rodlets were taken at 3 different viewing angles, $120^{\circ}$ apart. A picture of the carrier loaded with the four rodlets before image acquisition is shown in Figure 9. The neutron radiographs, obtained with both thermal and epithermal neutrons, are shown in Figure 10 through Figure 12 for the three orientations. As already mentioned from the analysis of the gamma scanning result, rodlet R06 presented a missing fuel piece at the top of the fuel stack, visible from the neutron radiography. Other than considerable cracking of the first pellet in R04 and of the upper insulator discs in R08, no additional unusual features could be observed in any of the orientations. 


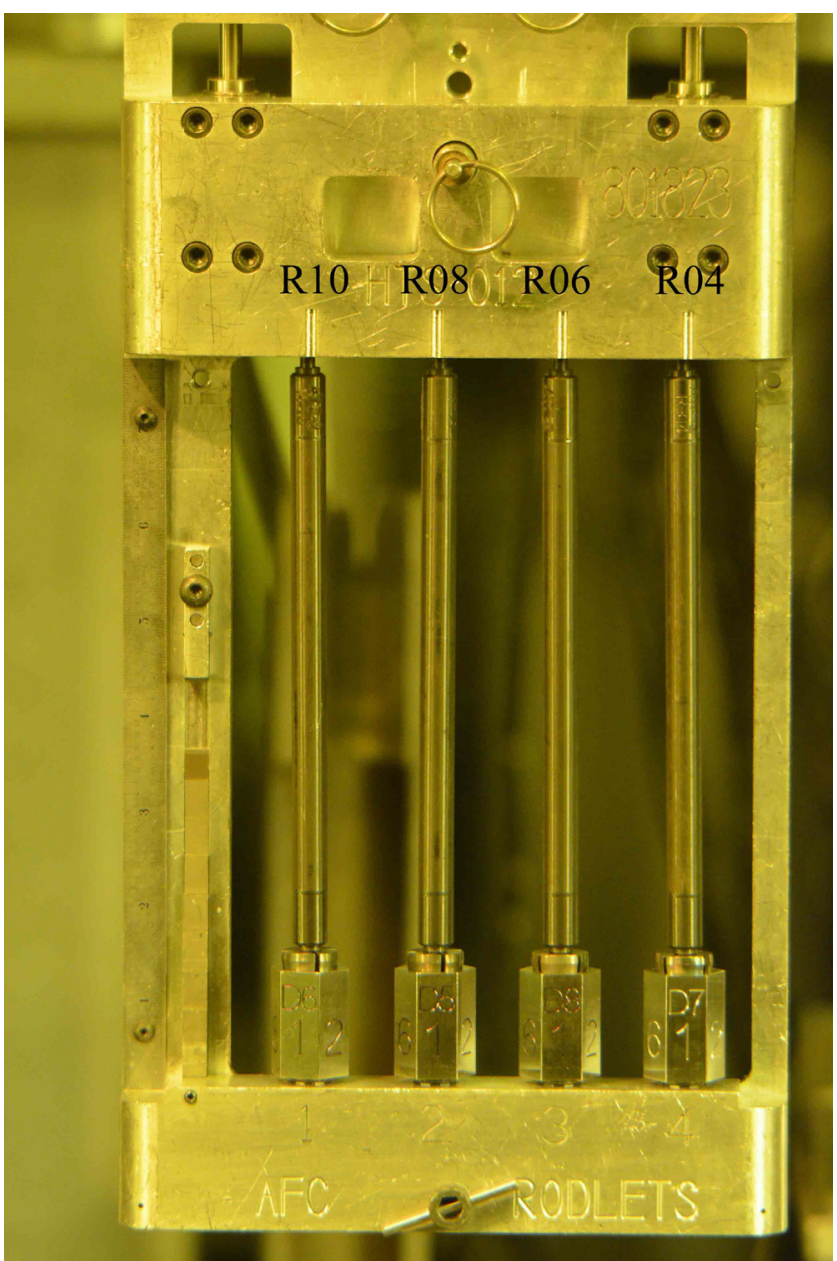

Figure 9. Carrier loaded with the four rodlets. 


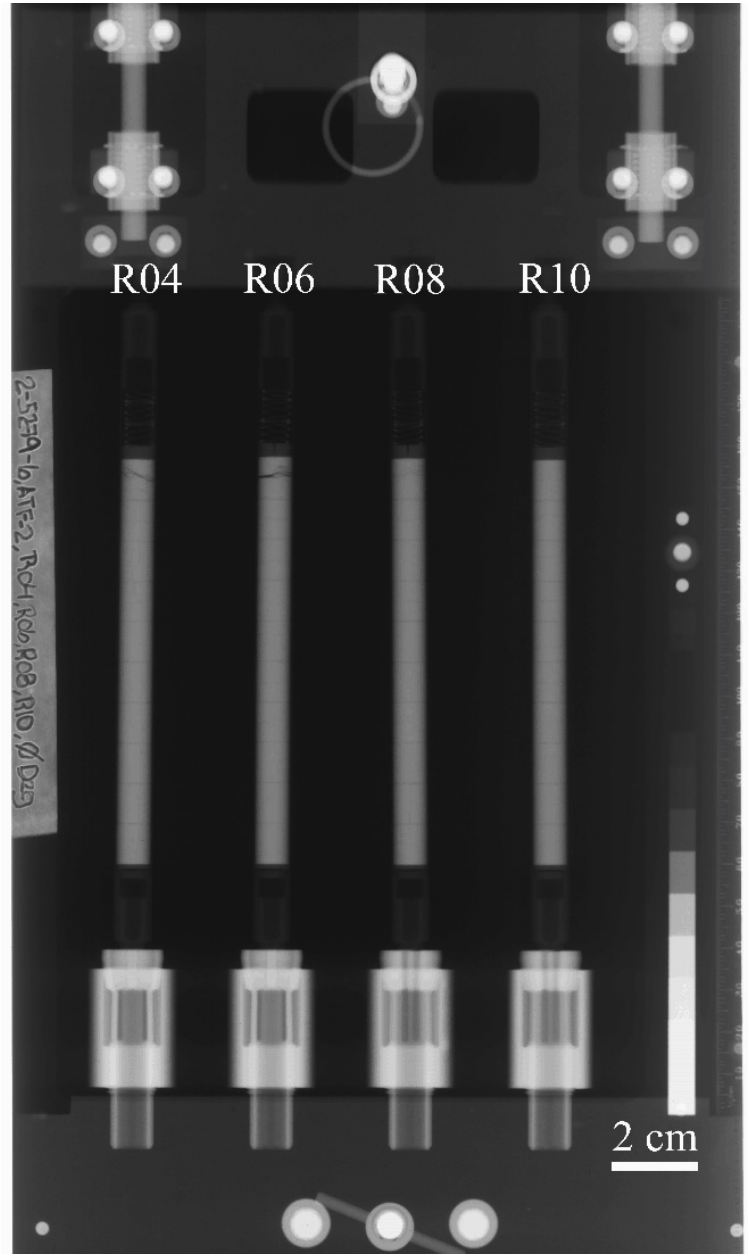

(a)

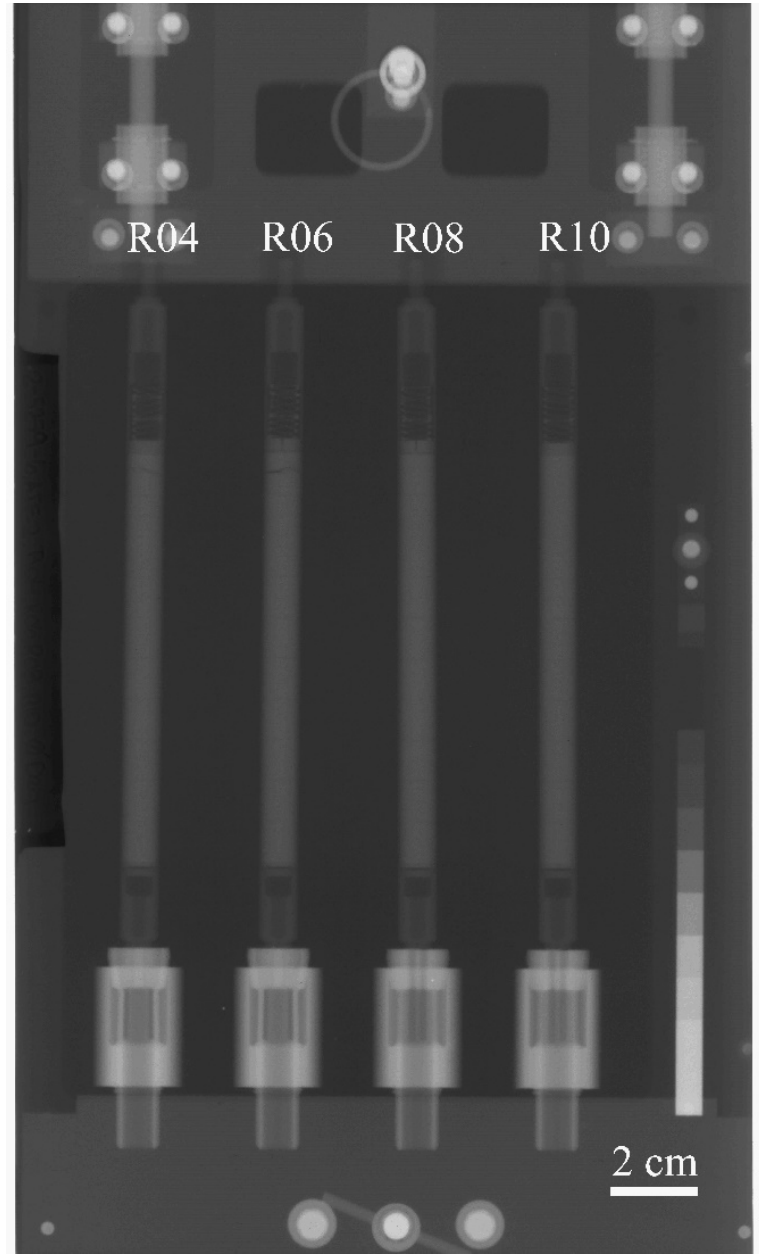

(b)

Figure 10. (a) Thermal and (b) epithermal neutron radiography of the rodlets at viewing angle $0^{\circ}$. 


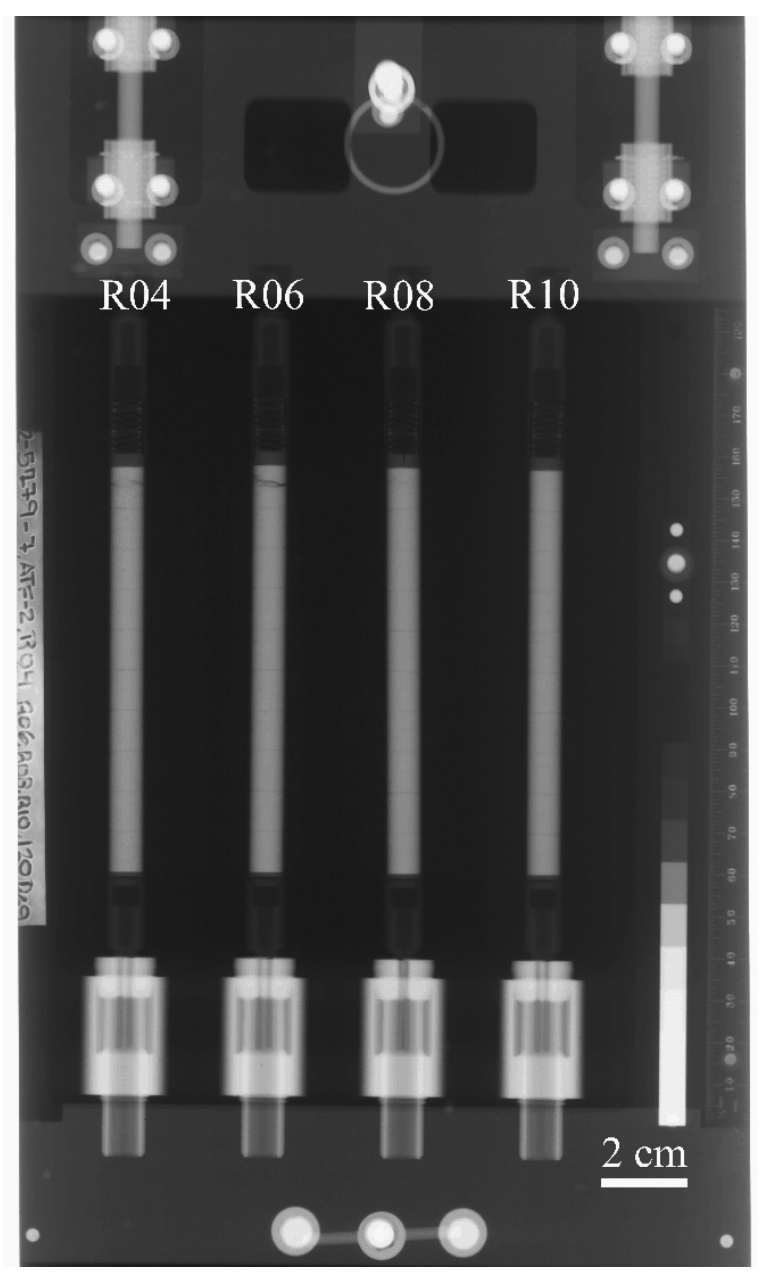

(a)

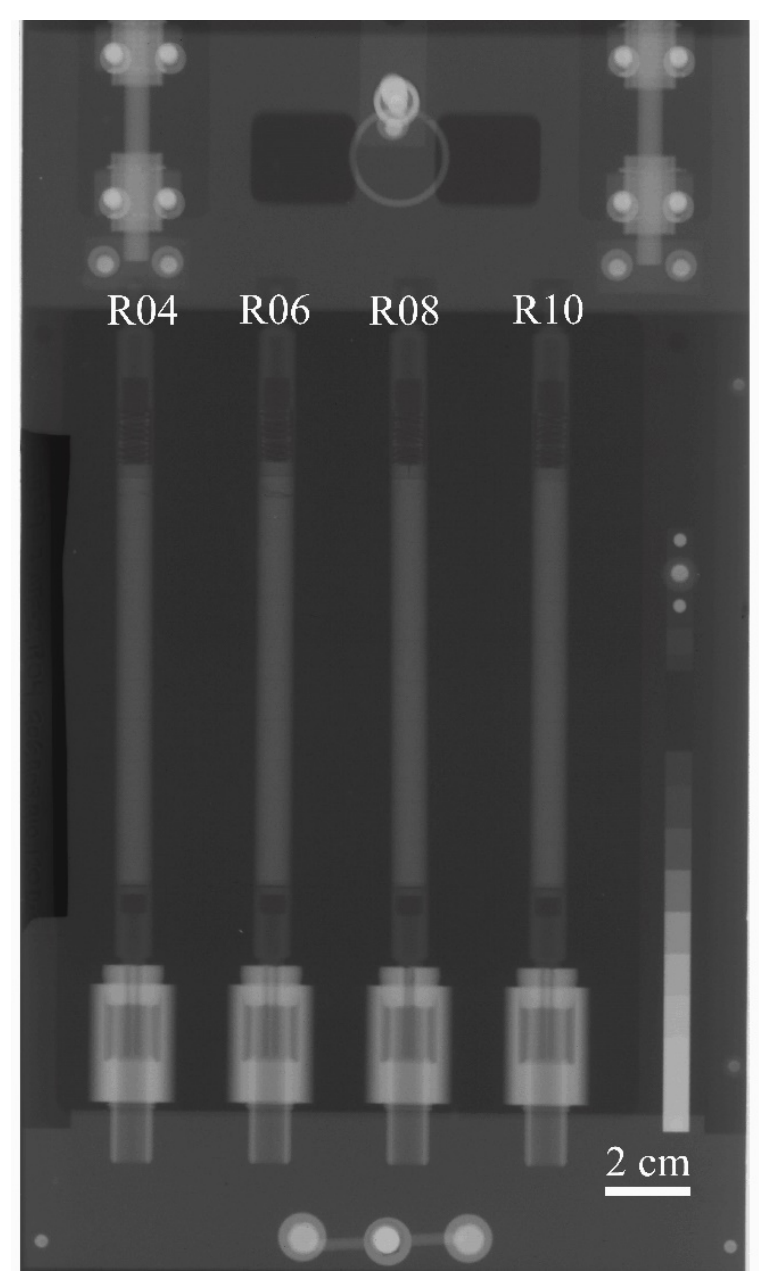

(b)

Figure 11. (a) Thermal and (b) epithermal neutron radiography of the rodlets at viewing angle $120^{\circ}$. 


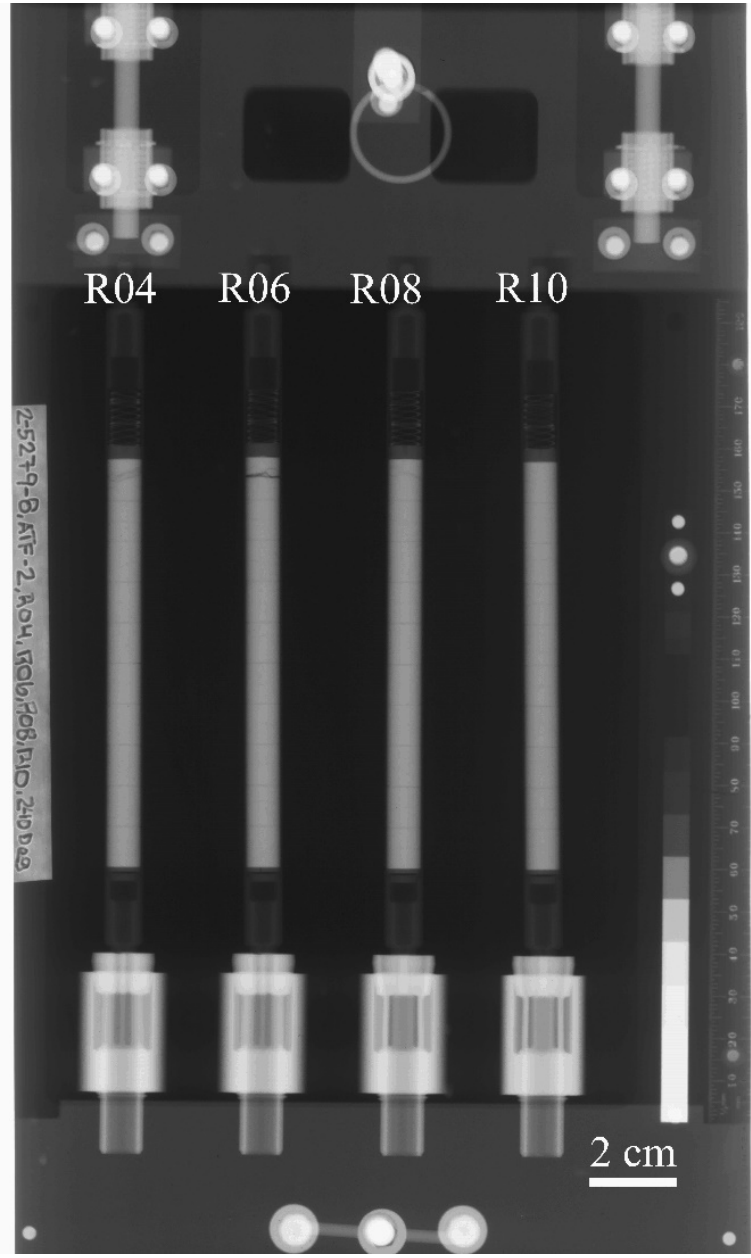

(a)

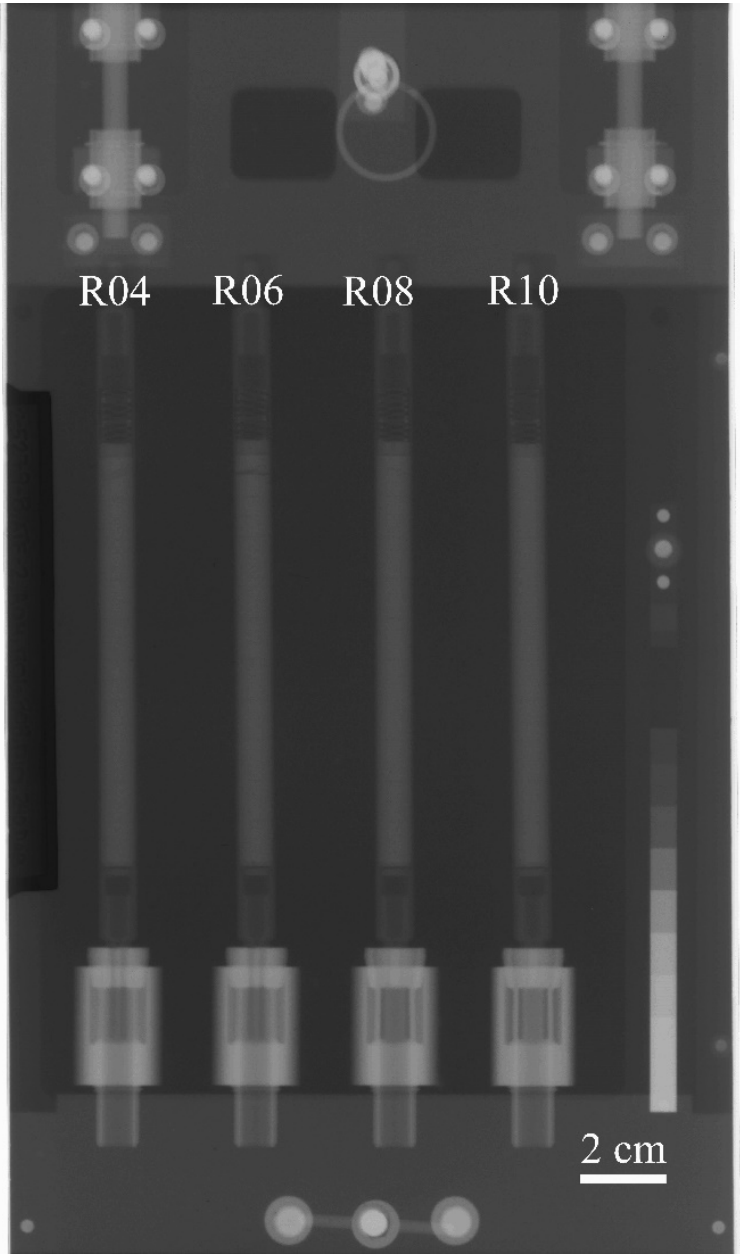

(b)

Figure 12. (a) Thermal and (b) epithermal neutron radiography of the rodlets at viewing angle $240^{\circ}$.

\subsection{Dimensional Inspection}

Dimensional inspection of the rodlets was carried out using a dimensional inspection device in HFEF. Outside diameter measurements were collected all along the rodlets in roughly $0.5 \mathrm{~mm}$ increments and at 36 angles every $5^{\circ}$ from the initial scan angle to $180^{\circ}$. Diameter measurements were collected with $\pm 5 \mu \mathrm{m}$ accuracy. The measurements at each axial location were averaged across the different angles. The results are shown in Figure 13 for R04, Figure 14 for R06, Figure 15 for R08, and Figure 16 for R10. The measured cladding dimension is within fabrication tolerance for each of the rodlets; hence, it is difficult to assess whether creep down could have occurred. At the axial location corresponding to the missing fuel piece in rodlet R06 spotted by gamma scanning and neutron radiography, no enhanced variation of the cladding outer dimension was measured. 


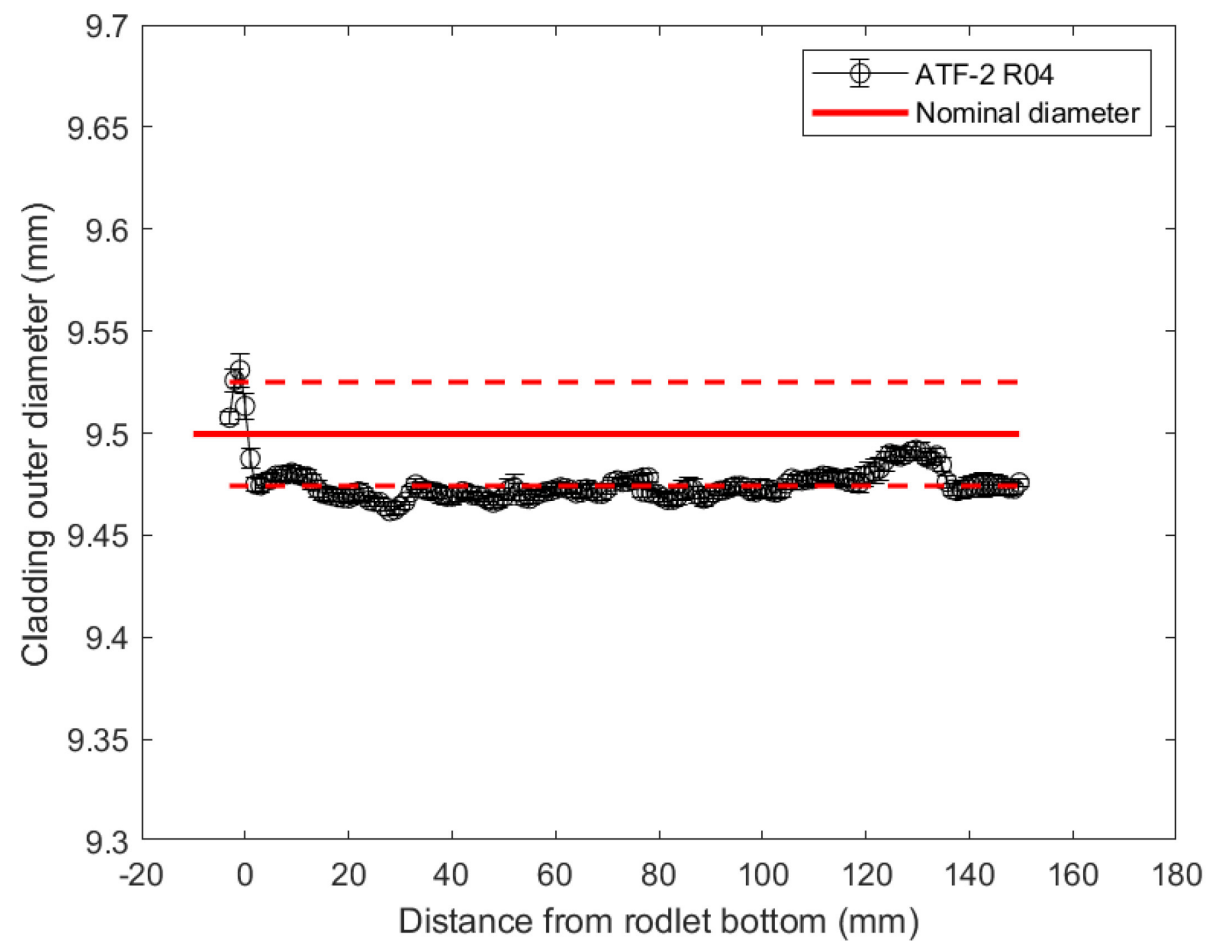

Figure 13. R04 cladding measured outer diameter (black circles). The solid red line represents the nominal diameter, while the dashed lines indicate the fabrication tolerance. 


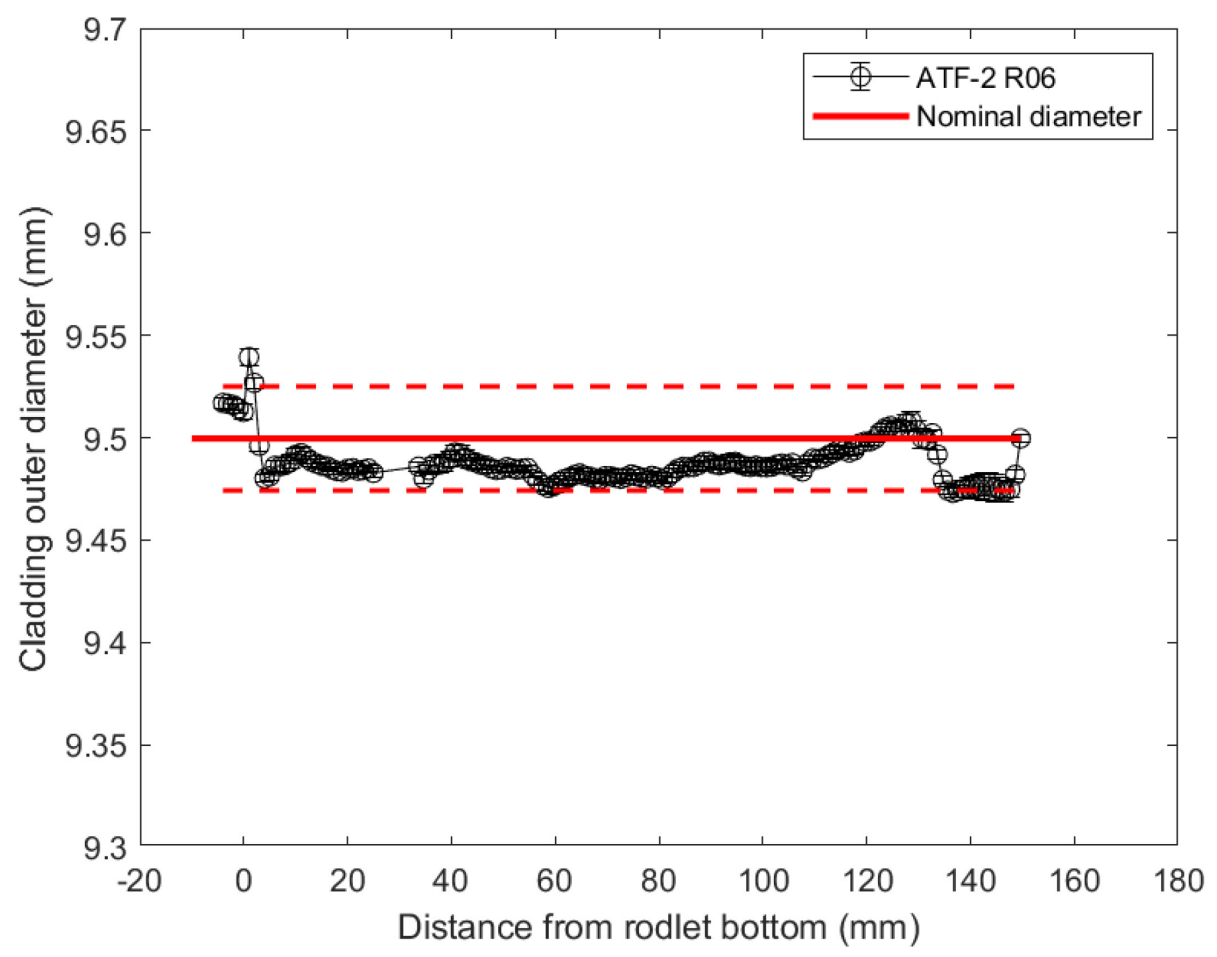

Figure 14. R06 cladding measured outer diameter (black circles). The solid red line represents the nominal diameter, while the dashed lines indicate the fabrication tolerance. 


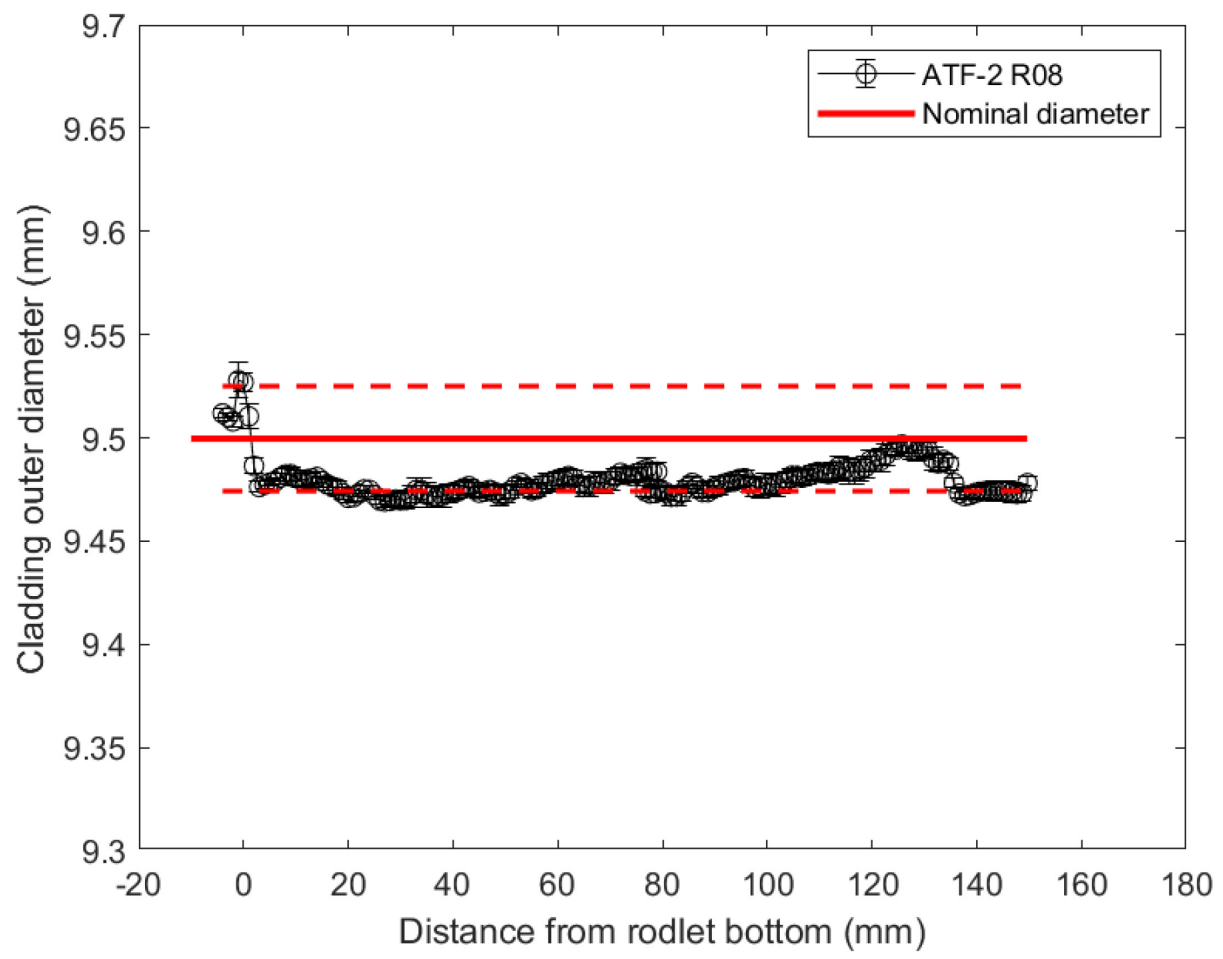

Figure 15. R08 cladding measured outer diameter (black circles). The solid red line represents the nominal diameter, while the dashed lines indicate the fabrication tolerance. 


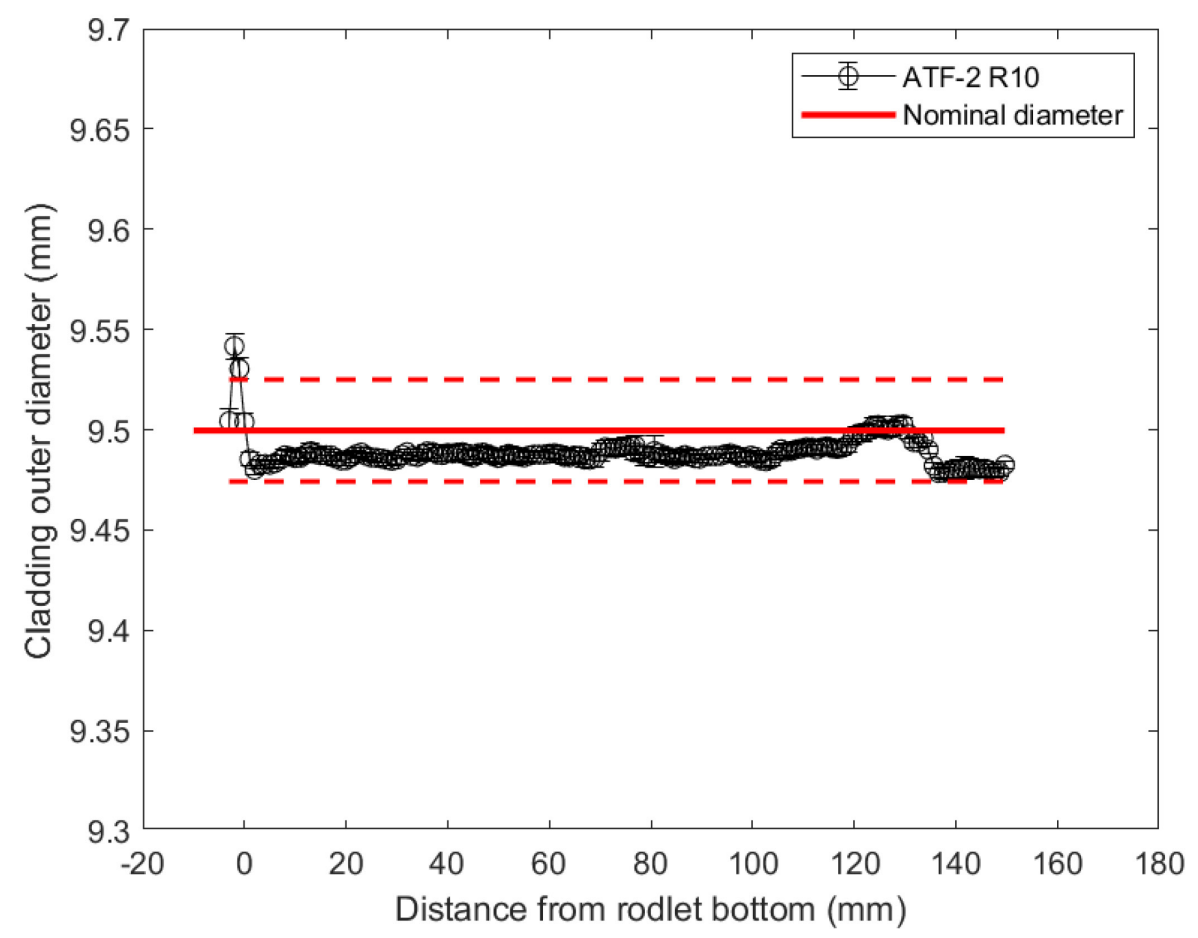

Figure 16. R10 cladding measured outer diameter (black circles). The solid red line represents the nominal diameter, while the dashed lines indicate the fabrication tolerance.

\section{CONCLUSIONS}

Seven baseline ATF-2 rodlets were discharged from ATR at the end of 2019 and were transported to the hot cells. The average discharge burnup of the seven rodlets is predicted to be in the range 8-10 $\mathrm{GWd} / \mathrm{tHM}$.

The rodlets underwent visual examination and gamma spectroscopy. In a second stage, four out of the seven rodlets were selected for further examinations. Profilometry and neutron radiography were executed to complete the initial dataset.

The visual exams showed presence of scratching which was caused by abrasion from rodlets support in the test train. Distribution of fission products analyzed by gamma spectrometry followed the neutron flux profile. No axial redistribution of any of the detectable gamma emitters was measured. A missing or displaced fuel piece was highlighted in rod R06 at the top of the fuel column by means of gamma spectrometry, and confirmed non-destructively by the neutron radiography. Other than this occurrence, no unusual features were observed in any of the rodlets. The cladding outer diameter remained within fabrication tolerance for all the rodlets. None of the examinations revealed behavior outside of what is considered standard performance for low burnup conventional LWR fuel. 


\section{REFERENCES}

[1] D. Kamerman, ATF-R Experiment Execution Plan, 2020.

[2] B. Curnutt, ATF-2 Cycle 166A As-Run and Payload Source Term for 10 Rodlet Shipment, Idaho National Laboratory, 2020.

[3] J.M. Harp, F. Cappia, L. Capriotti, Postirradiation Examination of the ATF-1 Experiments - 2018 Status, United States, 2018. doi:10.2172/1484529.

[4] F. Cappia, J.M. Harp, Post-Irradiation Examination of the ATF-1 Experiments - 2019 Status, 2019.

[5] D.A. Brown, et al, ENDF/B-VIII.0: The 8th Major Release of the Nuclear Reaction Data Library with CIELO-project Cross Sections, New Standards and Thermal Scattering Data, Nucl. Data Sheets. 148 (2018) 1-142. doi:https://doi.org/10.1016/j.nds.2018.02.001.

[6] H.D. Medema, K.M. Richardson, Advanced Fuels Campaign 2018 Accomplishments, United States, 2018. doi:10.2172/1515020. 